\title{
Modular Verification of Assembly Code with Stack-Based Control Abstractions
}

\author{
Xinyu Feng $^{\dagger}$ Zhong Shao $^{\dagger} \quad$ Alexander Vaynberg $^{\dagger} \quad$ Sen Xiang ${ }^{\ddagger} \quad$ Zhaozhong Ni $^{\dagger}$ \\ $\dagger^{\dagger}$ Department of Computer Science \\ Yale University \\ New Haven, CT 06520-8285, U.S.A. \\ ffeng, shao, alv, ni-zhaozhong\}@cs.yale.edu \\ \#Department of Computer Science and Technology \\ University of Science and Technology of China \\ Hefei, Anhui 230026, China \\ xiangsen@ustc.edu
}

\begin{abstract}
Runtime stacks are critical components of any modern softwarethey are used to implement powerful control structures such as function call/return, stack cutting and unwinding, coroutines, and thread context switch. Stack operations, however, are very hard to reason about: there are no known formal specifications for certifying C-style setjmp/longjmp, stack cutting and unwinding, or weak continuations (in C--). In many proof-carrying code (PCC) systems, return code pointers and exception handlers are treated as general first-class functions (as in continuation-passing style) even though both should have more limited scopes.

In this paper we show that stack-based control abstractions follow a much simpler pattern than general first-class code pointers. We present a simple but flexible Hoare-style framework for modular verification of assembly code with all kinds of stackbased control abstractions, including function call/return, tail call, setjmp/longjmp, weak continuation, stack cutting, stack unwinding, multi-return function call, coroutines, and thread context switch. Instead of presenting a specific logic for each control structure, we develop all reasoning systems as instances of a generic framework. This allows program modules and their proofs developed in different PCC systems to be linked together. Our system is fully mechanized. We give the complete soundness proof and a full verification of several examples in the Coq proof assistant.
\end{abstract}

\section{Introduction}

Runtime stacks are critical components of any modern softwarethey are used to implement powerful control structures such as procedure call/return, tail call [34, 8], C-style set jmp/longjmp [20], stack cutting and unwinding (for handling exceptions) [7, 12, 30], coroutines [10], and thread context switch [15]. Correct implementation of these constructs is of utmost importance to the safety and reliability of many software systems.

Stack-based controls, however, can be unsafe and error-prone. For example, both stack cutting and longjmp allow cutting across a chain of stack frames and returning immediately from a deeply nested function call. If not done carefully, it can invoke an obsolete longjmp or a dead weak continuation [30]). Neither C nor C-- [30] provides any formal specifications for certifying setjmp/longjmp, stack cutting and unwinding, or weak continuations. In Java virtual machine and Microsoft's .NET IL, operations on native $\mathrm{C}$ stacks are not managed so they must be trusted.

Stack operations are very hard to reason about because they involve subtle low-level invariants: both return code pointers and exception handlers should have restricted scopes, yet they are often stored in memory or passed in registers - making it difficult to track their lifetime. For instance, the following $\mathrm{C}$ program is compiled into the MIPS assembly code shown in Figure 1:

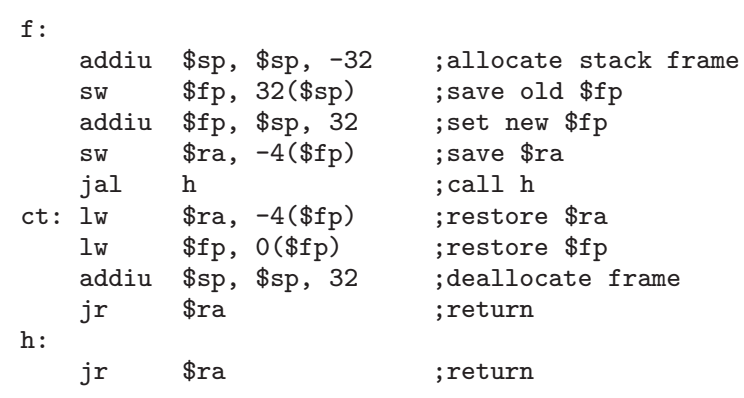

Figure 1. Stack-Based Function Call/Return

\begin{tabular}{|c|c|c|}
\hline void $f()\{$ & I & void $h()\{$ \\
\hline $\mathrm{h}()$ & 1 & return \\
\hline return; & 1 & \} \\
\hline
\end{tabular}

Before calling function $\mathrm{h}$, the caller $\mathrm{f}$ first saves its return code pointer (in \$ra) on the stack; the instruction jal $h$ loads the return address (the label ct) in $\$$ ra, and jumps to the label $h$; when $h$ returns, the control jumps back to the label ct, where $f$ restores its return code pointer and stack pointers and jumps back to its caller's code. The challenge is to formalize and capture the invariant that ct does not outlive $f$ even though it can escape into other functions.

Many proof-carrying code (PCC) systems [3, 14, 29] support stack-based controls by using continuation-passing style (CPS) [2]. CPS treats return addresses or exception handlers as first-class code pointers. Under CPS, the code following ct (lines 6-9) is treated not as a part of function $f$ but as a separate new function; when $h$ is called, the continuation function ct is passed as an extra argument in \$ra which is then called at the end of function h. CPS makes typechecking easier but it is still hard to describe the above invariant about $f$ and ct. Indeed, none of the existing PCC systems [26, $27,9,3,14]$ have successfully certified setjmp/longjmp, weak continuations, and general stack cutting and unwinding (see Sec 10 and $\mathrm{Sec} 2.1$ for an in-depth discussion about the related work).

In this paper we describe a formal system that can expose and validate the invariants of stack-based control abstractions. We show that return pointers (or exception handlers) are much more disciplined than general first-class code pointers. A return pointer is always associated with some logical control stack whose validity can be established statically. A function can cut to any return pointer if it can establish the validity of its associated logical control stack.

More specifically, we present a simple but flexible Hoare-style framework for modular verification of assembly code with all kinds of stack-based control abstractions (see Table 1). Instead of presenting a specific logic for each construct, we develop all reasoning systems as instances of a generic framework. This allows program modules and their proofs developed in different PCC systems to be 


\begin{tabular}{|l|l|l|}
\hline $\begin{array}{l}\text { Stack-Based } \\
\text { Control Abstraction }\end{array}$ & $\begin{array}{l}\text { Reasoning } \\
\text { System }\end{array}$ & $\begin{array}{l}\text { Definition \& } \\
\text { Formalization }\end{array}$ \\
\hline \hline function call/return & SCAP & SEC 4 \\
\hline tail call optimization $[34,8]$ & SCAP & SEC 4.4 \\
\hline exception: stack unwinding [30] & $\begin{array}{l}\text { SCAP-I } \\
\text { EUCAP }\end{array}$ & $\begin{array}{l}\text { SEC 5.1 } \\
\text { SEC 6.1 }\end{array}$ \\
\hline exception: stack cutting [30] & $\begin{array}{l}\text { SCAP-II } \\
\text { ECAP }\end{array}$ & $\begin{array}{l}\text { SEC 5.2 } \\
\text { SEC 6.2 }\end{array}$ \\
\hline multi-return function call [32] & SCAP-II & SEC 5.2 \\
\hline weak continuation [30] & SCAP-II & SEC 5.2 \\
\hline setjmp/longjmp [20] & SCAP-II & SEC 5.3 \\
\hline coroutines [10] & CAP-CR & SEC 7.1 \\
\hline coroutines + function call [10] & SCAP-CR & SEC 7.2 \\
\hline threads [15] & FCCAP & SEC 8 \\
\hline
\end{tabular}

Table 1. A Summary of Supported Control Abstractions

linked together. Our system is fully mechanized. We give the complete soundness proof and a full verification of several examples in the Coq proof assistant [35]. Our paper builds upon previous work on program verification but makes the following new contributions:

- As far as we know, our paper is the first to successfully formalize and verify sophisticated stack operations such as setjmp/longjmp, weak continuations, and general stack cutting. We verify raw assembly implementation so there is no loss of efficiency or additional runtime check. Our interface is simple, general, yet modular (so a library only needs to be verified once). Our framework is sound: a program certified using our system is free of unchecked runtime errors [20, 30].

- We have also done a thorough study of common stack-based control abstractions in the literatures (see Table 1). For each construct, we formalize its invariants and show how to certify its implementation. As an important advantage, all these systems are instances of a generic framework; in fact, the inference rules for each system are just derived lemmas in the base framework, so programs certified in different PCC systems can be linked together.

- Our SCAP system (Sec 4) is interesting and novel in its own right. Instead of treating return pointers as first-class code pointers (which require "impredicative types" [23, 29]), SCAP specifies the invariant at each program point using a pair of a precondition and a "local" guarantee (which states the obligation that the current function must fulfill before it can return or throw an exception). These guarantees, when chained together, is used to specify the logical control stack. SCAP is also orthogonal to the recent work on XCAP [29]: it can apply the same syntactic technique [29] to certify general first-class code pointers.

- Our certified framework is also very flexible. A logical control stack specifies a chain of valid return pointers, but it imposes no restriction on where we store these pointers. Because all invariants are specified as state predicates or state relations, we can support any physical stack layout and calling conventions.

In the rest of this paper, we first review common stack-based controls and summarize our main approach (Sec 2). We then define our machine platform and a generic Hoare-style framework (Sec 3). We present our SCAP system for certifying function call/return and show how to extend it to support different control abstractions in Table 1 (Sec 4-9). Finally we discuss implementation and related work, and then conclude.

\section{Background and Related Work}

Before giving an overview of our approach, we first survey common stack-based control abstractions in the literatures:
- Function call/return follow a strict "last-in, first-out" pattern: the callee always returns to the point where it was most recently called. Similar concepts include the JVM subroutines [22], which are used to compile the "try-finally" block in Java.

- The tail call optimization is commonly used in compiler implementation: if a function call occurs at the end of the current function, the callee will reuse the current stack frame and return directly to the caller of the current function.

- Exceptions, stack unwinding, and stack cutting. When an exception is raised, the control flow is transferred to the point at which the exception is handled. There are mainly two strategies for implementing exceptions (on stacks) [30]. Stack unwinding walks the stack one frame at a time until the handler is reached; intermediate frames contain a default handler that restores values of callee-save registers and re-raises the exception; a function always returns to the activation of its immediate caller. Stack cutting sets the stack pointer and the program counter directly to the handler which may be contained in a frame deep on the stack; intermediate frames are skipped over.

- Weak continuations and setjmp/longjmp. C-- uses weak continuations [30] to support different implementation strategies for exceptions. A weak continuation is similar to the first-class continuation except that it can only be defined inside a procedure and cannot outlive the activation of the enclosing procedure. $\mathrm{C}$ uses setjmp/longjmp library functions [20] to enable an immediate return from a deeply nested function call, the semantics of which is similar to weak-continuations (while the implementation may be more heavyweight). Especially, the function containing the setjmp must not have terminated when a longjmp is launched. Both C-- and $\mathrm{C}$ make no effort to prohibit invocation of a dead weak continuation or an obsolete longjmp.

- Multi-return function call. Shivers and Fisher [32] proposed MRLC to allow functions to have multiple return points, whose expressiveness sits between general CPS and first-order functions. The mechanism is similar to weak continuations, but proposed at a higher abstract level. Multi-return function call supports pure stack-based implementations.

- Coroutines and threads involve multiple execution contexts that exist concurrently. Control can be transferred from one execution context to another. Implementation of context switch does not follow the regular function calling convention: it fetches the return code pointer from the stack of the target coroutine (thread) and returns to the target instead of its caller.

\subsection{Reasoning about Control Abstractions}

Traditional Hoare-logic [16] uses the pre- and postcondition as specifications for programs. Most work on Hoare-logic [4] reasons about control structures in higher-level languages and does not directly reason about return code pointers in their semantics. To apply traditional Hoare-logic to generate mechanized proofs for low-level code, we need to first formalize auxiliary variables and the Invariance rule, which is a non-trivial issue and complicates the formalization, as shown in pervious work $[38,5]$; next, we need to relate the entry point with the exit point of a function and show the validity of return code pointers - this is hard at the assembly level due to the lack of abstractions.

Stata and Abadi [33] also observed two similar challenges for typechecking Java byte code subroutines. They propose a Hoarestyle type system to reason about subroutine calls ("jsr $L$ ") and returns ("ret $x$ "). To ensure the return address used by a subroutine is the one that is most recently pushed onto the stack, they have to disallow recursive function calls, and require labeling of code to relate the entry point with the return point of subroutines. 


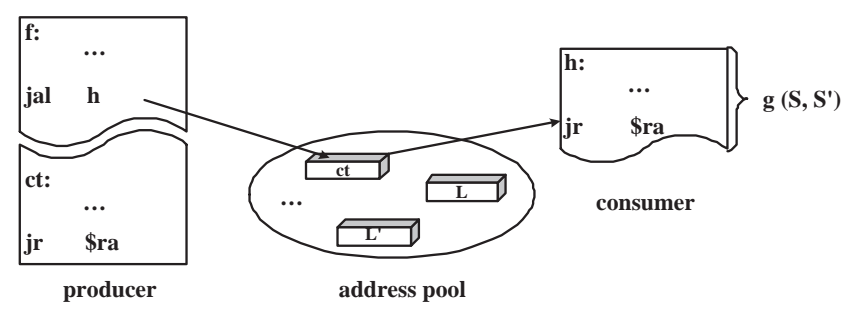

Figure 2. The Model for Code Pointers

Necula used Hoare triples to specify functions in SAL [26]. He needs a history $\mathcal{H}$ of states, which contains copies of the register file and the whole memory at the moment of function invocations. At the return point, the last state is popped up from $\mathcal{H}$ and the relation between that state and the current state is checked. Not a model of physical stacks, $\mathcal{H}$ is used purely for reasoning about function calls; it complicates the operational semantics of SAL significantly. Also, SAL uses a very restrictive physical stack model where only contiguous stack is supported and general pointer arguments (which may point into the stack) are not allowed.

To overcome the lack of structures in low-level code, many PCC systems have also used CPS to reason about regular control abstractions, which treats return code pointers (and exception handlers) as first-class code pointers. CPS is a general semantic model to support all the control abstractions above, but it is hard to use CPS to characterize the invariants of control stacks for specific control abstractions (e.g., setjmp/longjmp and weak continuation). CPS-based reasoning also requires specification of continuation pointers using "impredicative types" $[23,29])$, which makes the program specification complex and hard to understand. Another issue with CPS-based reasoning is the difficulty to specify first-class code pointers modularly in logic: because of the circular references between code pointers and data heap (which may in turn contains code pointers), it is not clear how to apply existing approaches $[25,3,29]$ to model sophisticated stack-based invariants.

\subsection{Our Approach}

In this paper we will show that we can support modular reasoning of stack-based control abstractions without treating them as firstclass code pointers. In our model, when a control transfer occurs, the pointer for the continuation code is deposited into an abstract "address pool" (which may be physically stored in memory or the register file). The code that saves the continuation is called a "producer", and the code that uses the continuation later is called a "consumer". In case of function calls, as shown in Figure 2, the caller is the "producer" and the callee is the "consumer", while the return address is the continuation pointer.

The producer is responsible for ensuring that each code pointer it deposits is a valid one and depositing the code pointer does not break the invariant of the address pool. The consumer ensures that the invariant established at its entry point still holds when it fetches the code pointer from the pool and makes an indirect jump. The validity of the code pointer is guaranteed by the invariant. To overcome the lack of abstraction at the assembly level, we use a guarantee g-a relation over a pair of states - to bridge the gap between the entry and exit points of the consumer. This approach avoids maintaining any state history or labeling of code.

The address pool itself is structureless, with each control abstraction molding the pool into the needed shape. For functions, exceptions, weak continuations, etc., the pool takes the form of a stack; for coroutines and threads it takes the form of a queue or a queue of stacks (each stack corresponding to a coroutine/thread). The invariant specified by a control abstraction also restricts how the pool is used. Function call, for example, restricts the (stack-

$$
\begin{aligned}
& (\text { Program }) \quad \mathbb{P} \quad::=(\mathbb{C}, \mathbb{S}, \mathbb{I}) \\
& \text { (CodeHeap) } \mathbb{C}::=\{\mathbf{f} \leadsto \mathbb{I}\}^{*} \\
& (\text { State }) \quad \mathbb{S} \quad::=(\mathbb{H}, \mathbb{R}) \\
& \text { (Heap) } \mathbb{H} \quad::=\{\mathrm{I} \leadsto \mathrm{w}\}^{*} \\
& \text { (RegFile) } \mathbb{R}::=\{\mathrm{r} \leadsto \mathrm{w}\}^{*} \\
& \text { (Register) } \mathrm{r} \quad::=\left\{\mathrm{r}_{k}\right\}^{k \in\{0 \ldots 31\}} \\
& \text { (Labels) } \mathrm{f}, 1::=i \text { (nat nums) } \\
& \text { (Word) } \quad \text { w }::=n \text { (integers) } \\
& \left(\text { InstrSeq) } \mathbb{I} \quad::=\mathrm{c} ; \mathbb{I}|\mathrm{j} \mathbf{f}| \text { jal } \mathrm{f}, \mathrm{f}_{\text {ret }} \mid \text { jr } \mathrm{r}_{s}\right. \\
& \text { (Command) } \mathrm{C} \quad::=\text { addu } \mathrm{r}_{d}, \mathrm{r}_{s}, \mathrm{r}_{t} \mid \text { addiu } \mathrm{r}_{d}, \mathrm{r}_{s}, \mathrm{w} \\
& \text { | beq } r_{s}, r_{t}, \mathrm{f} \mid \text { bgtz } r_{s}, \mathbf{f} \mid \operatorname{lw} r_{t}, \mathrm{w}\left(r_{s}\right) \\
& \text { subu } r_{d}, r_{s}, r_{t} \mid \operatorname{sw} r_{t}, \mathrm{w}\left(r_{s}\right)
\end{aligned}
$$

Figure 3. Syntax of Target Machine TM

$\begin{array}{lll}\text { \$zero } & r_{0} & \text { always zero } \\ \text { \$at } & r_{1} & \text { assembler temporary } \\ \text { \$v0 }-\$ \mathrm{v} 1 & r_{2}-r_{3} & \text { return values } \\ \text { \$a0 }-\$ a 3 & r_{4}-r_{7} & \text { arguments } \\ \text { \$t0 }-\$ \mathrm{t} 9 & r_{8}-r_{15}, r_{24}-r_{25} & \text { temporary (caller saved) } \\ \text { \$s0 }-\$ \mathrm{~s} 7 & r_{16}-r_{23} & \text { callee saved } \\ \text { \$k0 }-\$ \mathrm{k} 1 & r_{26}-r_{27} & \text { kernel } \\ \text { \$gp } & r_{28} & \text { global pointer } \\ \text { \$sp } & r_{29} & \text { stack pointer } \\ \text { \$fp } & r_{30} & \text { frame pointer } \\ \text { \$ra } & r_{31} & \text { return address }\end{array}$

Figure 4. Register Aliases and Usage

shaped) pool to a strict "last-in, first-out" pattern, and makes sure that all addresses remain constant until they are fetched.

In the rest of this paper, we will describe the invariant for each control abstraction. We also present a set of lemmas that allow programmers to verify structureless assembly code with higherlevel abstractions. Before we define these systems, we first present our generic CAP0 framework. All the systems for specific control abstractions will be presented as a set of lemmas in CAP0.

\section{The CAP0 Framework}

In this section, we first present a MIPS-style "untyped" target machine language (TM) and its operational semantics. Then we propose a general logic, CAP0, for verifying TM programs. The generic CAP0 framework will serve as the common basis for the interoperability of different logics.

\subsection{The Target Machine}

In Figure 3 we show the definition of a MIPS-style target machine (TM). A machine state is called a "Program" $(\mathbb{P})$, which consists of a read-only code heap $(\mathbb{C})$, an updatable state $(\mathbb{S})$, and an instruction sequence $(\mathbb{I})$. The code heap is a finite partial mapping from code labels to instruction sequences. The state $\mathbb{S}$ contains a data heap $(\mathbb{H})$ and a register file $(\mathbb{R})$. Each instruction sequence is a basic code block, i.e., a list of instructions ending with a jump-instruction. We use an instruction sequence $\mathbb{I}$ in $\mathbb{P}$ (rather than a program counter) to represent the basic block that is being executed.

The target machine has 32 registers. Following the MIPS convention, Figure 4 shows the register aliases and usage. All the assembly code shown in the rest of the paper follows this convention.

The instruction set captures the most basic and common MIPS instructions. Since we do not have a program counter, we change the syntax of the jal instruction and require that the return address be explicitly given. The execution of TM programs is modeled as a small-step transition from one program to another, i.e., $\mathbb{P} \longmapsto \mathbb{P}^{\prime}$. Figure 5 defines the program transition function. The semantics of 


\begin{tabular}{|c|c|}
\hline if $\mathbb{I}=$ & then $(\mathbb{C},(\mathbb{H}, \mathbb{R}), \mathbb{I}) \longmapsto$ \\
\hline$\overline{j \mathrm{jf}}$ & " $(\mathbb{C},(\mathbb{H}, \mathbb{R}), \mathbb{C}(\mathrm{f}))$ when $\mathrm{f} \in \operatorname{dom}(\mathbb{C})$ \\
\hline jal $f, f_{r e t}$ & $\left(\mathbb{C},\left(\mathbb{H}, \mathbb{R}\left\{\mathbf{r}_{31} \leadsto \mathrm{f}_{\text {ret }}\right\}\right), \mathbb{C}(\mathrm{f})\right)$ when $\mathrm{f} \in \operatorname{dom}(\mathbb{C})$ \\
\hline $\mathrm{jr} \mathrm{r}_{s}$ & $\left(\mathbb{C},(\mathbb{H}, \mathbb{R}), \mathbb{C}\left(\mathbb{R}\left(\mathrm{r}_{s}\right)\right)\right)$ when $\mathbb{R}\left(\mathrm{r}_{s}\right) \in \operatorname{dom}(\mathbb{C})$ \\
\hline beq $r_{s}, r_{t}, f ; \mathbb{I}^{\prime}$ & $\begin{array}{l}\left(\mathbb{C},(\mathbb{H}, \mathbb{R}), \mathbb{I}^{\prime}\right) \text { when } \mathbb{R}\left(\mathrm{r}_{s}\right) \neq \mathbb{R}\left(\mathrm{r}_{t}\right) \\
(\mathbb{C},(\mathbb{H}, \mathbb{R}), \mathbb{C}(\mathrm{f})) \text { when } \mathbb{R}\left(\mathrm{r}_{s}\right)=\mathbb{R}\left(\mathrm{r}_{t}\right), \mathrm{f} \in \operatorname{dom}(\mathbb{C})\end{array}$ \\
\hline bgtz $r_{S}, f ; \mathbb{I}^{\prime}$ & $\begin{array}{l}\left(\mathbb{C},(\mathbb{H}, \mathbb{R}), \mathbb{I}^{\prime}\right) \text { when } \mathbb{R}\left(\mathrm{r}_{s}\right) \leq 0 ; \\
(\mathbb{C},(\mathbb{H}, \mathbb{R}), \mathbb{C}(\mathrm{f})) \text { when } \mathbb{R}\left(\mathrm{r}_{s}\right)>0, \mathrm{f} \in \operatorname{dom}(\mathbb{C})\end{array}$ \\
\hline$c ; \mathbb{I}^{\prime}$ & $\left(\mathbb{C}, \operatorname{Next}_{\mathrm{c}}(\mathbb{H}, \mathbb{R}), \mathbb{I}^{\prime}\right)$ \\
\hline
\end{tabular}

where

\begin{tabular}{|l|c|}
\hline if $\mathrm{c}=$ & then $\operatorname{Next}_{\mathrm{c}}(\mathbb{H}, \mathbb{R})=$ \\
\hline \hline addu $\mathrm{r}_{d}, \mathrm{r}_{s}, \mathrm{r}_{t}$ & $\left(\mathbb{H}, \mathbb{R}\left\{\mathrm{r}_{d} \sim \mathbb{R}\left(\mathrm{r}_{s}\right)+\mathbb{R}\left(\mathrm{r}_{t}\right)\right\}\right)$ \\
\hline addiu $\mathrm{r}_{d}, \mathrm{r}_{s}, \mathrm{w}$ & $\left(\mathbb{H}, \mathbb{R}\left\{\mathrm{r}_{d} \sim \mathbb{R}\left(\mathrm{r}_{s}\right)+\mathrm{w}\right\}\right)$ \\
\hline Iw $\mathrm{r}_{t}, \mathrm{w}\left(\mathrm{r}_{s}\right)$ & $\left(\mathbb{H}, \mathbb{R}\left\{\mathrm{r}_{t} \sim \mathbb{H}\left(\mathbb{R}\left(\mathrm{r}_{s}\right)+\mathrm{w}\right)\right\}\right)$ \\
& when $\mathbb{R}\left(r_{s}\right)+\mathrm{w} \in \operatorname{dom}(\mathbb{H})$ \\
\hline subu $\mathrm{r}_{d}, \mathrm{r}_{s}, \mathrm{r}_{t}$ & $\left(\mathbb{H}, \mathbb{R}\left\{\mathrm{r}_{d} \sim \mathbb{R}\left(\mathrm{r}_{s}\right)-\mathbb{R}\left(\mathrm{r}_{t}\right)\right\}\right)$ \\
\hline sw $\mathrm{r}_{t}, \mathrm{w}\left(\mathrm{r}_{s}\right)$ & $\left(\mathbb{H}\left\{\mathbb{R}\left(\mathrm{r}_{s}\right)+\mathrm{w} \sim \mathbb{R}\left(\mathrm{r}_{t}\right)\right\}, \mathbb{R}\right)$ \\
& when $\mathbb{R}\left(\mathrm{r}_{s}\right)+\mathrm{w} \in \operatorname{dom}(\mathbb{H})$ \\
\hline
\end{tabular}

Figure 5. Operational Semantics of TM

most instructions are the same with corresponding MIPS instructions, except that code labels in jump-instructions (e.g., j f, jr $r$ ) and branch-instructions (e.g., beq $\left.r_{s}, r_{t}, f\right)$ are treated as absolute addresses instead of relative addresses.

\subsection{The CAP0 Framework}

CAP0 generalizes our previous work on CAP systems [40, 29]. It leaves the program specification unspecified, which can be customized to embed different logics into the framework. The soundness of CAP0 is independent of specific forms of program specifications. The framework supports separate verification of program modules using different verification logics.

\subsubsection{Program Specifications}

The verification constructs are defined as follows.

$$
\begin{array}{rll}
(\text { CdHpSpec }) & \Psi & ::=\{\mathrm{f} \sim \theta\}^{*} \\
(\text { CdSpec }) & \theta & ::=\ldots \\
(\text { Interp. }) & \text { a, } \llbracket \theta \rrbracket,\langle\mathrm{a}\rangle_{\Psi} & \in \text { CdHpSpec } \rightarrow \text { State } \rightarrow \text { Prop }
\end{array}
$$

To verify a program, the programmer needs to first give a specification $\Psi$ of the code heap, which is a finite mapping from code labels to code specifications $\theta$. To support different verification methodology, the CAP0 framework does not enforce the form of $\theta$. Instead, it requires the programmer to provide an interpretation function [-_]] which maps $\theta$ to predicates (a) over the code heap specification and the program state. CAP0 uses the interpretation of code specifications as its assertion language.

To support separate verification of modules, we add an extra constraint on the arguments of a using the lifting function $\left\langle_{-}\right\rangle_{\Psi}$, which says that the specification $\Psi$ of the local module is the smallest set of code specifications we need to know to verify this module. The lifting function is defined as follows:

$$
\langle\mathrm{a}\rangle_{\Psi} \triangleq \lambda \Psi^{\prime} \cdot \lambda \mathbb{S} .\left(\Psi \subseteq \Psi^{\prime}\right) \wedge \mathrm{a} \Psi^{\prime} \mathbb{S} .
$$

We will give a detailed explanation of CAP0's support of modularity in the next section.

\subsubsection{Inference Rules and Soundness}

We use the following judgments to define inference rules:

$$
\begin{aligned}
& \Psi \vdash\{\text { a }\} \mathbb{P} \quad \text { (well-formed program) } \\
& \Psi \vdash \mathbb{C}: \Psi^{\prime} \quad \text { (well-formed code heap) } \\
& \vdash\{\mathrm{a}\} \mathbb{I} \quad \text { (well-formed instruction sequence) }
\end{aligned}
$$

Figure 6 shows the inference rules of CAP0.

$$
\begin{aligned}
& \Psi \vdash\{\mathrm{a}\} \mathbb{P} \quad \text { (Well-formed Program) } \\
& \frac{\Psi_{\mathrm{G}} \vdash \mathbb{C}: \Psi_{\mathrm{G}}\left(\mathrm{a} \Psi_{\mathrm{G}} \mathbb{S}\right) \vdash\{\mathrm{a}\} \mathbb{I}}{\Psi_{\mathrm{G}} \vdash\{\mathrm{a}\}(\mathbb{C}, \mathbb{S}, \mathbb{I})} \\
& \Psi \vdash \mathbb{C}: \Psi^{\prime} \quad \text { (Well-formed Code Heap) } \\
& \frac{\mathrm{a}=\llbracket \theta \rrbracket \quad \vdash\left\{\langle\mathrm{a}\rangle_{\Psi_{\mathrm{L}}}\right\} \mathbb{I}}{\Psi_{\mathrm{L}} \vdash\{\mathrm{f} \leadsto \mathbb{I}\}:\{\mathrm{f} \sim \theta\}} \\
& \Psi_{1} \vdash \mathbb{C}_{1}: \Psi_{1}^{\prime} \quad \Psi_{2} \vdash \mathbb{C}_{2}: \Psi_{2}^{\prime} \quad \operatorname{dom}\left(\mathbb{C}_{1}\right) \cap \operatorname{dom}\left(\mathbb{C}_{2}\right)=\emptyset \\
& \forall \mathrm{f} \in \operatorname{dom}\left(\Psi_{1}\right) \cap \operatorname{dom}\left(\Psi_{2}\right) . \Psi_{1}(\mathrm{f})=\Psi_{2}(\mathrm{f}) \\
& \Psi_{1} \cup \Psi_{2} \vdash \mathbb{C}_{1} \cup \mathbb{C}_{2}: \Psi_{1}^{\prime} \cup \Psi_{2}^{\prime} \\
& \frac{\forall \Psi, \mathbb{S} \text {. a } \Psi \mathbb{S} \rightarrow \llbracket \Psi(\mathrm{f}) \rrbracket \Psi \mathbb{S}}{\vdash\{\mathrm{a}\} \mathrm{j} \mathrm{f}} \\
& \frac{\forall \Psi, \mathbb{H}, \mathbb{R} . \text { a } \Psi(\mathbb{H}, \mathbb{R}) \rightarrow \llbracket \Psi(\mathrm{f}) \rrbracket \Psi\left(\mathbb{H}, \mathbb{R}\left\{\mathrm{ra} \sim \mathrm{f}_{\text {ret }}\right\}\right)}{\vdash\{\mathrm{a}\} \mathrm{jal} \mathbf{f}, \mathbf{f}_{\text {ret }}} \\
& \frac{\forall \Psi, \mathbb{S} \text {. a } \Psi \mathbb{S} \rightarrow \llbracket \Psi\left(\mathbb{S} . \mathbb{R}\left(\mathrm{r}_{s}\right)\right) \rrbracket \Psi \mathbb{S}}{\vdash\{\mathrm{a}\} \mathrm{jr} \mathrm{r}_{S}} \\
& \vdash\left\{\mathbf{a}^{\prime}\right\} \mathbb{I} \\
& \forall \Psi, \mathbb{S} . \text { a } \Psi \mathbb{S} \rightarrow\left(\left(\mathbb{S} . \mathbb{R}\left(r_{s}\right) \neq \mathbb{S} . \mathbb{R}\left(r_{t}\right) \rightarrow \mathrm{a}^{\prime} \Psi \mathbb{S}\right) \wedge\right. \\
& \begin{array}{l}
\left.\left(\mathbb{S} . \mathbb{R}\left(r_{s}\right)=\mathbb{S} . \mathbb{R}\left(r_{t}\right) \rightarrow \llbracket \Psi(\mathrm{f}) \rrbracket \Psi \mathbb{S}\right)\right) \\
\vdash\{\text { a }\} \text { beq } r_{s}, r_{t}, f ; \mathbb{I}
\end{array} \\
& \vdash\left\{\mathbf{a}^{\prime}\right\} \mathbb{I} \\
& \forall \Psi, \mathbb{S} . \mathrm{a} \Psi \mathbb{S} \rightarrow\left(\left(\mathbb{S} . \mathbb{R}\left(r_{s}\right) \leq 0 \rightarrow \mathrm{a}^{\prime} \Psi \mathbb{S}\right) \wedge\right. \\
& \left.\left(\mathbb{S} . \mathbb{R}\left(r_{s}\right)>0 \rightarrow \llbracket \Psi(f) \rrbracket \Psi \mathbb{S}\right)\right)
\end{aligned}
$$

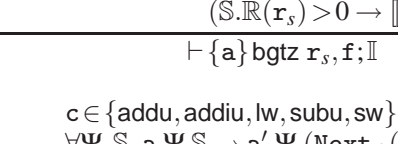

$$
\begin{aligned}
& \frac{\forall \Psi, \mathbb{S} . \mathrm{a} \Psi \mathbb{S} \rightarrow \mathrm{a}^{\prime} \Psi\left(\operatorname{Next}_{\mathrm{c}}(\mathbb{S})\right) \quad \vdash\left\{\mathrm{a}^{\prime}\right\} \mathbb{I}}{\vdash\{\mathrm{a}\} \mathrm{c} ; \mathbb{I}}
\end{aligned}
$$$$
\text { (PROG) }
$$

$$
\text { Figure 6. Inference Rules for CAP0 }
$$

A program is well-formed (the PROG rule) if there exists a global code heap specification $\Psi_{\mathrm{G}}$ and an assertion a such that:

- the global code heap $\mathbb{C}$ is well-formed with respect to $\Psi_{\mathrm{G}}$;

- given $\Psi_{\mathrm{G}}$, the current state $\mathbb{S}$ satisfies the assertion a; and

- the current instruction sequence $\mathbb{I}$ is well-formed.

The CAP0 framework supports separate verification of program modules. Modules are modeled as small code heaps which contain at least one code block. The specification of a module contains not only specifications of the code blocks in the current module, but also specifications of external code blocks which will be called by the module. In the judgment $\Psi \vdash \mathbb{C}: \Psi^{\prime}, \Psi$ contains specifications for imported external code and for code within the module $\mathbb{C}$ (to support recursive functions), while $\Psi^{\prime}$ contains specifications for exported interfaces for other modules. Programmers are required to first establish the well-formedness of each individual module via the CDHP rule. Two non-intersecting well-formed modules can then be linked together via the LINK rule. The PROG rule requires that all modules be linked into a well-formed global code heap.

In the CDHP rule, the user specification $\theta$ (for $\mathbb{I}$ ) is first mapped to a predicate over the code heap specification and the program state, and then lifted by the lifting function parameterized by the local specification $\Psi_{\mathrm{L}}$ of this module. Later on, we will see that none of the instruction rules (e.g., J and JAL) refer to the global program specification $\Psi_{\mathrm{G}}$. Instead, a universally quantified $\Psi$ is 
used with the constraint that it must be a superset of $\Psi_{\mathrm{L}}$. Such a constraint is enforced by the lifting function $\left\langle_{-}\right\rangle_{\mathrm{L}}$.

The well-formedness of instruction sequences ensures that it is safe to execute $\mathbb{I}$ in a machine state satisfying the assertion a. An instruction sequence beginning with $c$ is safe (rule SEQ) if we can find an assertion $a^{\prime}$ which serves both as the postcondition of $c$ (that is, $a^{\prime}$ holds on the updated machine state after executing c, as captured by the implication) and as the precondition of the tail instruction sequence. A direct jump is safe (rule $\mathrm{J}$ ) if the current assertion can imply the assertion of the target code block as specified in $\Psi$. Rules for other jump and branch instructions are similar to the $\mathrm{J}$ rule. When proving the well-formedness of an instruction sequence, a programmer's task includes applying the appropriate inference rules and finding intermediate assertions such as $\mathrm{a}^{\prime}$.

Soundness The soundness of CAP0 inference rules with respect to the operational semantics of TM is established following the syntactic approach [39] to prove type soundness. We do not require the specific form of code specifications $\theta$ to prove the soundness.

Lemma 3.1 (Progress) If $\Psi \vdash\{a\} \mathbb{P}$, then there exists a program $\mathbb{P}^{\prime}$, such that $\mathbb{P} \longmapsto \mathbb{P}^{\prime}$.

Lemma 3.2 (Preservation) If $\Psi \vdash\{a\} \mathbb{P}$, and $\mathbb{P} \longmapsto \mathbb{P}^{\prime}$, then there exists $a^{\prime}, \Psi \vdash\left\{a^{\prime}\right\} \mathbb{P}^{\prime}$.

Theorem 3.3 (Soundness) If $\Psi \vdash\{\mathrm{a}\} \mathbb{P}$, then for all natural number $n$, there exists a program $\mathbb{P}^{\prime}$ such that $\mathbb{P} \longmapsto{ }^{n} \mathbb{P}^{\prime}$.

We have formally encoded the soundness proof [36] in the Coq proof assistant.

CAPO and Previous CAP systems. The CAP0 framework is a generalization of our previous work on CAP systems [40, 29]. The original CAP [40] does not support separate verification of program modules. The idea of letting assertions be parameterized by $\Psi$ and using universally-quantified $\Psi$ in the CAP0 inference rules, is borrowed from Ni and Shao's work on XCAP [29]. XCAP is proposed to reason about general first-class code pointers, where a special form of assertions (with type State $\rightarrow$ Prop $X$ ) is used for program specifications.

CAP0 generalizes XCAP and leaves the form of program specifications unspecified. The interpretation function in CAP0, which is different from the one in XCAP, maps different forms of specifications to a general form. It is trivial to embed the original CAP in CAP0 by the following customization and interpretation.

$$
\begin{aligned}
& \text { (Assertion) } \mathrm{p} \in \text { State } \rightarrow \text { Prop } \\
& \text { (CdSpec) } \quad \theta \quad::=\mathrm{p} \\
& \text { (Interp.) } \llbracket \mathrm{p} \rrbracket \triangleq \lambda \Psi . \lambda \mathbb{S} . \mathrm{p} \mathbb{S}
\end{aligned}
$$

XCAP and its extension [28] for weak memory update can be embedded into CAP0 too if we use formulae of type (State $\rightarrow$ PropX) to customize the $\theta$ in CAP0, and use the interpretation in XCAP as our interpretation function. TAL [24] may also be embedded in CAP0 indirectly through XCAP, as shown by Ni and Shao [28].

\section{SCAP for Function Call/Return}

\subsection{Stack-Based Reasoning for Function Call}

We present SCAP as an instance of the CAP0 framework. The code specification $\theta$ in CAP0 is instantiated with the SCAP specification, which is defined as:

$$
\begin{aligned}
& (\text { Assertion) } \mathrm{p} \in \text { State } \rightarrow \text { Prop } \\
& \text { (Guarantee) } \mathrm{g} \in \text { State } \rightarrow \text { State } \rightarrow \text { Prop } \\
& \text { (CdSpec) } \theta::=(\mathrm{p}, \mathrm{g})
\end{aligned}
$$

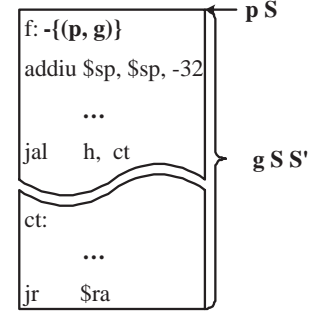

(a)

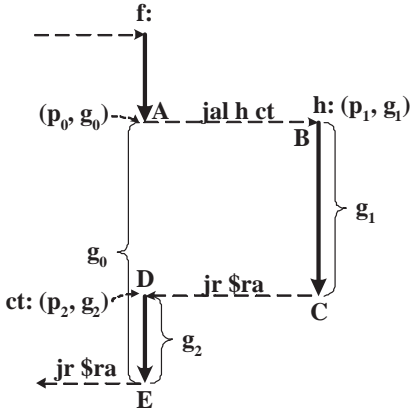

(b)
Figure 7. The Model for Function Call/Return in SCAP

A precondition for an instruction sequence contains a predicate $\mathrm{p}$ specifying the current state, and a guarantee $\mathrm{g}$ describing the relation between the current state and the state at the return point of the current function (if the function ever returns). Figure 7(a) shows the meaning of the specification $(\mathrm{p}, \mathrm{g})$ for the function $f$ defined in Figure 1 (Section 1). Note that $g$ may cover multiple instruction sequences. If a function has multiple return points, g governs all the traces from the current program point to any return point.

Figure 7(b) illustrates a function call to $h$ from $f$ at point $A$, with the return address $c t$. The specification of $h$ is $\left(p_{1}, g_{1}\right)$. Specifications at $A$ and $D$ are $\left(\mathrm{p}_{0}, \mathrm{~g}_{0}\right)$ and $\left(\mathrm{p}_{2}, \mathrm{~g}_{2}\right)$ respectively, where $\mathrm{g}_{0}$ governs the code segment A-E and $\mathrm{g}_{2}$ governs $\mathrm{D}-\mathrm{E}$.

To ensure that the program behaves correctly, we need to enforce the following conditions:

- the precondition of function $\mathrm{h}$ can be satisfied, i.e.,

$$
\forall \mathbb{H}, \mathbb{R} \cdot \mathrm{p}_{0}(\mathbb{H}, \mathbb{R}) \rightarrow \mathrm{p}_{1}(\mathbb{H}, \mathbb{R}\{\text { \$ra } \leadsto \mathrm{ct}\}) ;
$$

- after $\mathrm{h}$ returns, $\mathrm{f}$ can resume its execution from point $\mathrm{D}$, i.e.,

$$
\forall \mathbb{H}, \mathbb{R}, \mathbb{S}^{\prime} \cdot \mathrm{p}_{0}(\mathbb{H}, \mathbb{R}) \rightarrow \mathrm{g}_{1}(\mathbb{H}, \mathbb{R}\{\$ \mathrm{ra} \sim \mathrm{ct}\}) \mathbb{S}^{\prime} \rightarrow \mathrm{p}_{2} \mathbb{S}^{\prime} ;
$$

- if the function $\mathrm{h}$ and the code segment D-E satisfy their specifications, the specification for $\mathrm{A}-\mathrm{E}$ is satisfied, i.e.,

$$
\begin{aligned}
& \forall \mathbb{H}, \mathbb{R}, \mathbb{S}^{\prime}, \mathbb{S}^{\prime \prime} \cdot \mathrm{p}_{0}(\mathbb{H}, \mathbb{R}) \rightarrow \\
& \quad \mathrm{g}_{1}(\mathbb{H}, \mathbb{R}\{\$ \mathrm{ra} \sim \mathrm{ct}\}) \mathbb{S}^{\prime} \rightarrow \mathrm{g}_{2} \mathbb{S}^{\prime} \mathbb{S}^{\prime \prime} \rightarrow \mathrm{g}_{0}(\mathbb{H}, \mathbb{R}) \mathbb{S}^{\prime \prime} ;
\end{aligned}
$$

- the function $\mathrm{h}$ must reinstate the return code pointer when it returns, i.e., $\forall \mathbb{S}, \mathbb{S}^{\prime} . \mathrm{g}_{1} \mathbb{S} \mathbb{S}^{\prime} \rightarrow \mathbb{S} . \mathbb{R}(\$ \mathrm{ra})=\mathbb{S}^{\prime} . \mathbb{R}(\$ \mathrm{ra})$.

Above conditions are enforced by the CALL rule shown in Figure 8 (ignore the meaning of $[[(p, g)]]$ for the time being, which will be defined later).

To check the well-formedness of an instruction sequence beginning with $\mathrm{c}$, the programmer needs to find an intermediate specification $\left(\mathrm{p}^{\prime}, \mathrm{g}^{\prime}\right)$, which serves both as the postcondition for $\mathrm{c}$ and as the precondition for the remaining instruction sequence. As shown in the SCAP-SEQ rule, we check that:

- the remaining instruction sequence is well-formed with regard to the intermediate specification;

- $\mathrm{p}^{\prime}$ is satisfied by the resulting state of $\mathrm{c}$; and

- if the remaining instruction sequence satisfies its guarantee $\mathrm{g}^{\prime}$, the original instruction sequence satisfies $g$.

Suppose the state transition sequence made by the function is $\left(\mathbb{S}_{0}, \ldots, \mathbb{S}_{n}\right)$. To show that the function satisfies its guarantee $\mathrm{g}$ (i.e., $\mathrm{g} \mathbb{S}_{0} \mathbb{S}_{n}$ ), we enforce the following chain of implication relations:

$$
\mathrm{g}_{n} \mathbb{S}_{n-1} \mathbb{S}_{n} \rightarrow \mathrm{g}_{n-1} \mathbb{S}_{n-2} \mathbb{S}_{n} \rightarrow \ldots \rightarrow \mathrm{g} \mathbb{S}_{0} \mathbb{S}_{n},
$$

where each $g_{i}$ is the intermediate specification used at each verification step. Each arrow on the chain is enforced by rules such as SCAP-SEQ. The head of the chain (i.e., $\mathrm{g}_{n} \mathbb{S}_{n-1} \mathbb{S}_{n}$ ) is enforced 


$$
\begin{aligned}
& \mathbf{f}, \mathbf{f}_{r e t} \in \operatorname{dom}\left(\Psi_{\mathrm{L}}\right) \quad\left(\mathrm{p}^{\prime}, \mathrm{g}^{\prime}\right)=\Psi_{\mathrm{L}}(\mathbf{f}) \quad\left(\mathrm{p}^{\prime \prime}, \mathrm{g}^{\prime \prime}\right)=\Psi_{\mathrm{L}}\left(\mathbf{f}_{\text {ret }}\right) \\
& \forall \mathbb{H}, \mathbb{R} \cdot \mathrm{p}(\mathbb{H}, \mathbb{R}) \rightarrow \mathrm{p}^{\prime}\left(\mathbb{H}, \mathbb{R}\left\{\$ \mathbf{r a} \sim \mathbf{f}_{r e t}\right\}\right) \\
& \forall \mathbb{H}, \mathbb{R}, \mathbb{S}^{\prime} . \mathrm{p}(\mathbb{H}, \mathbb{R}) \rightarrow \mathrm{g}^{\prime}\left(\mathbb{H}, \mathbb{R}\left\{\$ \mathrm{ra} \sim \mathrm{f}_{r e t}\right\}\right) \mathbb{S}^{\prime} \rightarrow \\
& \left(\mathrm{p}^{\prime \prime} \mathbb{S}^{\prime} \wedge\left(\forall \mathbb{S}^{\prime \prime} \cdot \mathrm{g}^{\prime \prime} \mathbb{S}^{\prime} \mathbb{S}^{\prime \prime} \rightarrow \mathrm{g}(\mathbb{H}, \mathbb{R}) \mathbb{S}^{\prime \prime}\right)\right) \\
& \forall \mathbb{S}, \mathbb{S}^{\prime} \cdot \mathrm{g}^{\prime} \mathbb{S} \mathbb{S}^{\prime} \rightarrow \mathbb{S} \cdot \mathbb{R}(\$ \mathrm{ra})=\mathbb{S}^{\prime} \cdot \mathbb{R}(\$ \mathrm{ra}) \\
& \vdash\left\{\langle\llbracket(\mathrm{p}, \mathrm{g}) \rrbracket\rangle \Psi_{\mathrm{L}}\right\} \text { jal } \mathbf{f}, \mathrm{f}_{\text {ret }} \\
& \mathrm{c} \in\{\text { addu, addiu, Iw, subu, sw }\} \\
& \vdash\left\{\left\langle\llbracket\left(\mathrm{p}^{\prime}, \mathrm{g}^{\prime}\right) \rrbracket\right\rangle \Psi_{\mathrm{L}}\right\} \mathbb{I} \quad \forall \mathbb{S} . \mathrm{p} \mathbb{S} \rightarrow \mathrm{p}^{\prime}\left(\operatorname{Next}_{\mathrm{C}}(\mathbb{S})\right) \\
& \forall \mathbb{S}, \mathbb{S}^{\prime} \cdot \mathrm{p} \mathbb{S} \rightarrow \mathrm{g}^{\prime}\left(\operatorname{Next}_{\mathrm{c}}(\mathbb{S})\right) \mathbb{S}^{\prime} \rightarrow \mathrm{g} \mathbb{S} \mathbb{S}^{\prime} \\
& \vdash\left\{\langle\llbracket(\mathrm{p}, \mathrm{g}) \rrbracket\rangle_{\Psi_{\mathrm{L}}}\right\} \mathrm{c} ; \mathbb{I} \\
& \frac{\forall \mathbb{S} . p \mathbb{S} \rightarrow \mathrm{g} \mathbb{S} \mathbb{S}}{\vdash\left\{\langle\llbracket(\mathrm{p}, \mathrm{g}) \rrbracket\rangle_{\Psi_{\mathrm{L}}}\right\} \mathrm{jr} \$ \mathrm{ra}} \\
& \mathrm{f} \in \operatorname{dom}\left(\Psi_{\mathrm{L}}\right) \quad\left(\mathrm{p}^{\prime}, \mathrm{g}^{\prime}\right)=\Psi_{\mathrm{L}}(\mathrm{f}) \\
& \frac{\forall \mathbb{S} . p \mathbb{S} \rightarrow \mathrm{p}^{\prime} \mathbb{S} \quad \forall \mathbb{S}, \mathbb{S}^{\prime} . \mathrm{p} \mathbb{S} \rightarrow \mathrm{g}^{\prime} \mathbb{S} \mathbb{S}^{\prime} \rightarrow \mathrm{g} \mathbb{S} \mathbb{S}^{\prime}}{\vdash\left\{\langle\llbracket(\mathrm{p}, \mathrm{g}) \rrbracket\rangle_{\Psi_{\mathrm{L}}}\right\} \mathrm{j} f} \\
& \mathrm{f} \in \operatorname{dom}\left(\Psi_{\mathrm{L}}\right) \quad\left(\mathrm{p}^{\prime \prime}, \mathrm{g}^{\prime \prime}\right)=\Psi_{\mathrm{L}}(\mathrm{f}) \quad \vdash\left\{\left\langle\llbracket\left(\mathrm{p}^{\prime}, \mathrm{g}^{\prime}\right) \rrbracket\right\rangle_{\Psi_{\mathrm{L}}}\right\} \mathbb{I} \\
& \forall \mathbb{S} . \mathrm{p} \mathbb{S} \rightarrow \mathbb{S} . \mathbb{R}\left(\mathrm{r}_{s}\right) \neq \mathbb{S} . \mathbb{R}\left(\mathrm{r}_{t}\right) \rightarrow\left(\mathrm{p}^{\prime} \mathbb{S} \wedge\left(\forall \mathbb{S}^{\prime} \cdot \mathrm{g}^{\prime} \mathbb{S} \mathbb{S}^{\prime} \rightarrow \mathrm{g} \mathbb{S} \mathbb{S}^{\prime}\right)\right) \\
& \frac{\forall \mathbb{S} . \mathrm{p} \mathbb{S} \rightarrow \mathbb{S} . \mathbb{R}\left(r_{S}\right)=\mathbb{S} . \mathbb{R}\left(r_{t}\right) \rightarrow\left(\mathrm{p}^{\prime \prime} \mathbb{S} \wedge\left(\forall \mathbb{S}^{\prime} \cdot \mathrm{g}^{\prime \prime} \mathbb{S} \mathbb{S}^{\prime} \rightarrow \mathrm{g} \mathbb{S} \mathbb{S}^{\prime}\right)\right)}{\vdash\left\{\langle\llbracket(\mathrm{p}, \mathrm{g}) \rrbracket\rangle_{\Psi_{\mathrm{L}}}\right\} \text { beq } r_{s}, r_{t}, \mathrm{f} ; \mathbb{I}} \\
& f \in \operatorname{dom}\left(\Psi_{\mathrm{L}}\right) \quad\left(\mathrm{p}^{\prime \prime}, \mathrm{g}^{\prime \prime}\right)=\Psi_{\mathrm{L}}(\mathrm{f}) \quad \vdash\left\{\left\langle\left[\left(\mathrm{p}^{\prime}, \mathrm{g}^{\prime}\right)\right]\right\rangle \Psi_{\mathrm{L}}\right\} \mathbb{I} \\
& \forall \mathbb{S} . p \mathbb{S} \rightarrow \mathbb{S} . \mathbb{R}\left(r_{s}\right) \leq 0 \rightarrow\left(\mathrm{p}^{\prime} \mathbb{S} \wedge\left(\forall \mathbb{S}^{\prime} \cdot \mathrm{g}^{\prime} \mathbb{S} \mathbb{S}^{\prime} \rightarrow \mathrm{g} \mathbb{S} \mathbb{S}^{\prime}\right)\right) \\
& \frac{\forall \mathbb{S} . \mathrm{p} \mathbb{S} \rightarrow \mathbb{S} . \mathbb{R}\left(\mathrm{r}_{s}\right)>0 \rightarrow\left(\mathrm{p}^{\prime \prime} \mathbb{S} \wedge\left(\forall \mathbb{S}^{\prime} . \mathrm{g}^{\prime \prime} \mathbb{S} \mathbb{S}^{\prime} \rightarrow \mathrm{g} \mathbb{S} \mathbb{S}^{\prime}\right)\right)}{\vdash\left\{\langle\llbracket(\mathrm{p}, \mathrm{g}) \rrbracket\rangle_{\Psi_{\mathrm{L}}}\right\} \text { bgtz } \mathrm{r}_{s}, \mathrm{f} ; \mathbb{I}}
\end{aligned}
$$

\section{Figure 8. SCAP Inference Rules}

by the RET rule (where $\mathbb{S}_{n-1}$ is the same with $\mathbb{S}_{n}$ since the jump instruction does not change the state), therefore we can finally reach the conclusion of $g \mathbb{S}_{0} \mathbb{S}_{n}$.

SCAP also supports tail function call, where the callee reuses the caller's stack frame and the return code pointer. To make a tail function call, the caller just directly jumps to the callee's code. As shown in the T-CALL rule, we need to check that the guarantee of the callee matches the guarantee that remains to be fulfilled by the caller function.

Rules for branch instructions are straightforward. The SCAPBGTZ rule is like a combination of the SCAP-SEQ rule and the TCALL rule, since the execution may either fall through or jump to the target code label, depending on whether the condition holds.

Notice that all the code specifications $\Psi_{\mathrm{L}}$ used in SCAP rules are the local specifications for the current module. SCAP supports modular reasoning about function call/return in the sense that caller and callee can be in different modules and be certified separately. When specifying the callee function, we do not need any knowledge about the return address \$ra in its precondition $\mathrm{p}$. The RET rule for the instruction "jr \$ra" does not have any constraint on \$ra either. Examples in Section 4.4 illustrate how to write program specifications in SCAP.

\subsection{The Stack Invariant}

Figure 9 shows a snapshot of the stack of return continuations: the specification of the current function is $\left(\mathrm{p}_{0}, \mathrm{~g}_{0}\right)$, which will return to its caller at the end; and the caller will return to the caller's caller... The return continuations in the dashed box compose a logical control stack.

To establish the soundness of the SCAP inference rules, we need to ensure that when the current function returns at $\mathrm{A}$, \$ra contains a valid code pointer with the specification $\left(\mathrm{p}_{1}, \mathrm{~g}_{1}\right)$, and

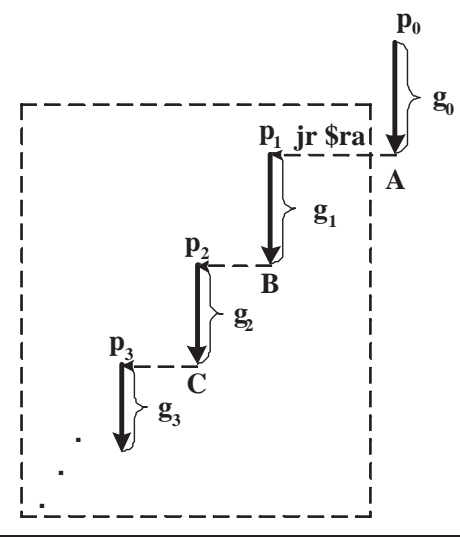

Figure 9. The Logical Control Stack

$\mathrm{p}_{1}$ is satisfied. Similarly we need to ensure that, at return points B and C, \$ra contains valid code pointers with specifications $\left(\mathrm{p}_{2}, \mathrm{~g}_{2}\right)$ and $\left(p_{3}, g_{3}\right)$ respectively, and that $p_{2}$ and $p_{3}$ are satisfied by then. Suppose the current state is $\mathbb{S}_{0}$ which satisfies $\mathrm{p}_{0}$, above safety requirement can be formalized as follows:

$$
\begin{aligned}
& \mathrm{g}_{0} \mathbb{S}_{0} \mathbb{S}_{1} \rightarrow \\
& \mathbb{S}_{1} \cdot \mathbb{R}(\$ \mathrm{ra}) \in \operatorname{dom}(\Psi) \wedge \Psi\left(\mathbb{S}_{1} \cdot \mathbb{R}(\$ \mathrm{ra})\right)=\left(\mathrm{p}_{1}, \mathrm{~g}_{1}\right) \wedge \mathrm{p}_{1} \mathbb{S}_{1} ; \\
& \mathrm{g}_{0} \mathbb{S}_{0} \mathbb{S}_{1} \rightarrow \mathrm{g}_{1} \mathbb{S}_{1} \mathbb{S}_{2} \rightarrow \\
& \mathbb{S}_{2} \cdot \mathbb{R}(\$ \mathrm{ra}) \in \operatorname{dom}(\Psi) \wedge \Psi\left(\mathbb{S}_{2} \cdot \mathbb{R}(\$ \mathrm{ra})\right)=\left(\mathrm{p}_{2}, \mathrm{~g}_{2}\right) \wedge \mathrm{p}_{2} \mathbb{S}_{2} ; \\
& \mathrm{g}_{0} \mathbb{S}_{0} \mathbb{S}_{1} \rightarrow \mathrm{g}_{1} \mathbb{S}_{1} \mathbb{S}_{2} \rightarrow \mathrm{g}_{2} \mathbb{S}_{2} \mathbb{S}_{3} \rightarrow \\
& \mathbb{S}_{3} \cdot \mathbb{R}(\$ \mathrm{ra}) \in \operatorname{dom}(\Psi) \wedge \Psi\left(\mathbb{S}_{3} \cdot \mathbb{R}(\$ \mathrm{ra})\right)=\left(\mathrm{p}_{3}, \mathrm{~g}_{3}\right) \wedge \mathrm{p}_{3} \mathbb{S}_{3} ;
\end{aligned}
$$

where $\Psi$ is the program specification, and each $\mathbb{S}_{i}$ is implicitly quantified by universal quantification.

Generalizing above safety requirement, we recursively define the "well-formed control stack with depth $n$ " as follows:

$$
\begin{aligned}
& \operatorname{WFST}(0, \mathrm{~g}, \mathbb{S}, \Psi) \triangleq \neg \exists \mathbb{S}^{\prime} \cdot \mathrm{g} \mathbb{S} \mathbb{S}^{\prime} \\
& \operatorname{WFST}(n, \mathrm{~g}, \mathbb{S}, \Psi) \triangleq \\
& \quad \forall \mathbb{S}^{\prime} \cdot \mathrm{g} \mathbb{S} \mathbb{S}^{\prime} \rightarrow \mathbb{S}^{\prime} \cdot \mathbb{R}(\$ \mathrm{ra}) \in \operatorname{dom}(\Psi) \wedge \mathrm{p}^{\prime} \mathbb{S}^{\prime} \wedge \operatorname{WFST}\left(n-1, \mathrm{~g}^{\prime}, \mathbb{S}^{\prime}, \Psi\right) \\
& \text { where }\left(\mathrm{p}^{\prime}, \mathrm{g}^{\prime}\right)=\Psi\left(\mathbb{S}^{\prime} \cdot \mathbb{R}(\$ \mathrm{ra})\right) .
\end{aligned}
$$

When the stack has depth 0 , we are in the outermost function which has no return code pointer (the program either "halts" or enters an infinite loop). In this case, we simply require that there exist no $\mathbb{S}^{\prime}$ at which the function can return, i.e., $\neg \exists \mathbb{S}^{\prime} . \mathrm{g} \mathbb{S} \mathbb{S}^{\prime}$.

Then the stack invariant we need to enforce is that, at each program point with specification $(p, g)$, the program state $\mathbb{S}$ must satisfy $p$ and there exists a well-formed control stack in $\mathbb{S}$. The invariant is formally defined as:

$$
\mathrm{p} \mathbb{S} \wedge \exists n \cdot \operatorname{WFST}(n, \mathrm{~g}, \mathbb{S}, \Psi) .
$$

Note here we do not care about the actual depth of the control stack.

To prove the soundness of SCAP, we need to prove that the invariant holds at every step of program execution. The stack invariant essentially explains why we can have such a simple RET rule, which "typechecks" the "jr \$ra" instruction without requiring that \$ra contain a valid code pointer.

\subsection{SCAP in the CAP Framework}

We prove the soundness of SCAP by showing that SCAP inference rules are provable from the corresponding $\mathrm{CAP} 0$ rules, given a proper interpretation function for the SCAP specifications.

In Section 4.1 we instantiated the CAP0 code specification $\theta$ with $(\mathrm{p}, \mathrm{g})$ in SCAP, without giving the interpretation function. Having defined the stack invariant, the interpretation of $(p, g)$ is 


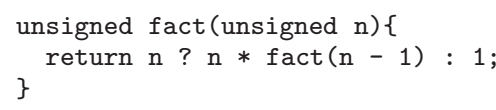

(a) regular recursive function

void fact (unsigned $* r$, unsigned $n$ ) \{ if $(\mathrm{n}==0)$ return;

$* \mathrm{r}=* \mathrm{r} * \mathrm{n}$;

\}

$f \operatorname{act}(r, n-1)$;

(b) tail recursion with pointer arguments

Figure 10. Factorial Functions in $\mathrm{C}$

$$
\begin{aligned}
& \text { TRUE } \triangleq \lambda \text { S.True } \\
& \operatorname{Hnid}(\mathrm{ls}) \triangleq \forall \mathrm{l} \notin \mathrm{ls} .[l]=[l]^{\prime} \\
& \operatorname{Frm}[i] \triangleq[[\$ \mathrm{fp}]-i] \\
& \text { NoG } \triangleq \lambda \mathbb{S} . \lambda \mathbb{S}^{\prime} \text {.False } \\
& \operatorname{Rid}(r s) \triangleq \forall r \in r s,[r]=[r]^{\prime} \\
& \operatorname{Frm}^{\prime}[i] \triangleq[[\$ \mathrm{fp}]-i]^{\prime} \\
& \mathrm{g}_{\mathrm{frm}} \triangleq[\$ \mathrm{sp}]^{\prime}=[\$ \mathrm{sp}]+3 \wedge[\$ \mathrm{fp}]^{\prime}=\operatorname{Frm}[0] \\
& \wedge[\$ \mathrm{ra}]^{\prime}=\operatorname{Frm}[1] \wedge[\$ \mathrm{~s} 0]^{\prime}=\operatorname{Frm}[2]
\end{aligned}
$$

Figure 11. Macros for SCAP Examples

simply defined as the invariant:

$$
[[(\mathrm{p}, \mathrm{g})]] \triangleq \lambda \Psi \cdot \lambda \mathbb{S} . \mathrm{p} \mathbb{S} \wedge \exists n \cdot \operatorname{WFST}(n, \mathrm{~g}, \mathbb{S}, \Psi) .
$$

The proof of SCAP inference rules as lemmas in CAP0 are presented in Appendix A and encoded in Coq [36].

\subsection{Examples}

In this section we show how SCAP can be used to support calleesave registers, optimizations for tail-recursions, and general pointer arguments in $\mathrm{C}$.

Figure 10 shows two versions of the factorial function implemented in $\mathrm{C}$. The first one is a regular recursive function, while the second one saves the intermediate result in the address passed as argument and makes a tail-recursive call.

The compiled assembly code of these two functions is shown in Figure 12 and 13. In both programs, the label entry points to the initial code segment where the function fact is called. SCAP specifications for the code heap are embedded in the code, enclosed by -\{\} . Figure 11 shows definitions of macros used in the code specifications. To simplify the presentation, we use $[r]$ and $[1]$ to represent values contained in the register $r$ and memory location 1. We also use primed representations $[r]^{\prime}$ and $[1]^{\prime}$ to represent values in the resulting state (the second argument) of a guarantee $g$. $\operatorname{Rid}(r s)$ means all the registers in $r s$ are preserved by the function. $\mathrm{Hnid}(\mathrm{l}$ s) means all memory cells except those with addresses in $1 \mathrm{~s}$ are preserved. Frm $[i]$ represents the $i^{t h}$ word on the stack frame.

The specification at the entrance point (labeled by prolog) of the first function is given as (TRUE, $\left.\mathrm{g}_{0}\right)$ in Figure 12. The precondition defines no constraint on the value of \$ra. The guarantee $g_{0}$ specifies the behavior of the function:

- the return value $[\$ \mathrm{v} 0]$ is the factorial of the argument $[\$ \mathrm{a} 0]$;

- callee-save registers are not updated; and

- the memory, other than the stack frames, are not updated.

If we use pre-/post-conditions in traditional Hoare-Logic to specify the function, we have to use auxiliary variables to specify the first point, and apply the Invariance Rule for the last two points. Using the guarantee $\mathrm{g}_{0}$ they can be easily expressed.

In the second implementation (in Figure 13), the caller passes the address of a stack variable to the function fact. The tail recursion is optimized by reusing the stack frame and making a

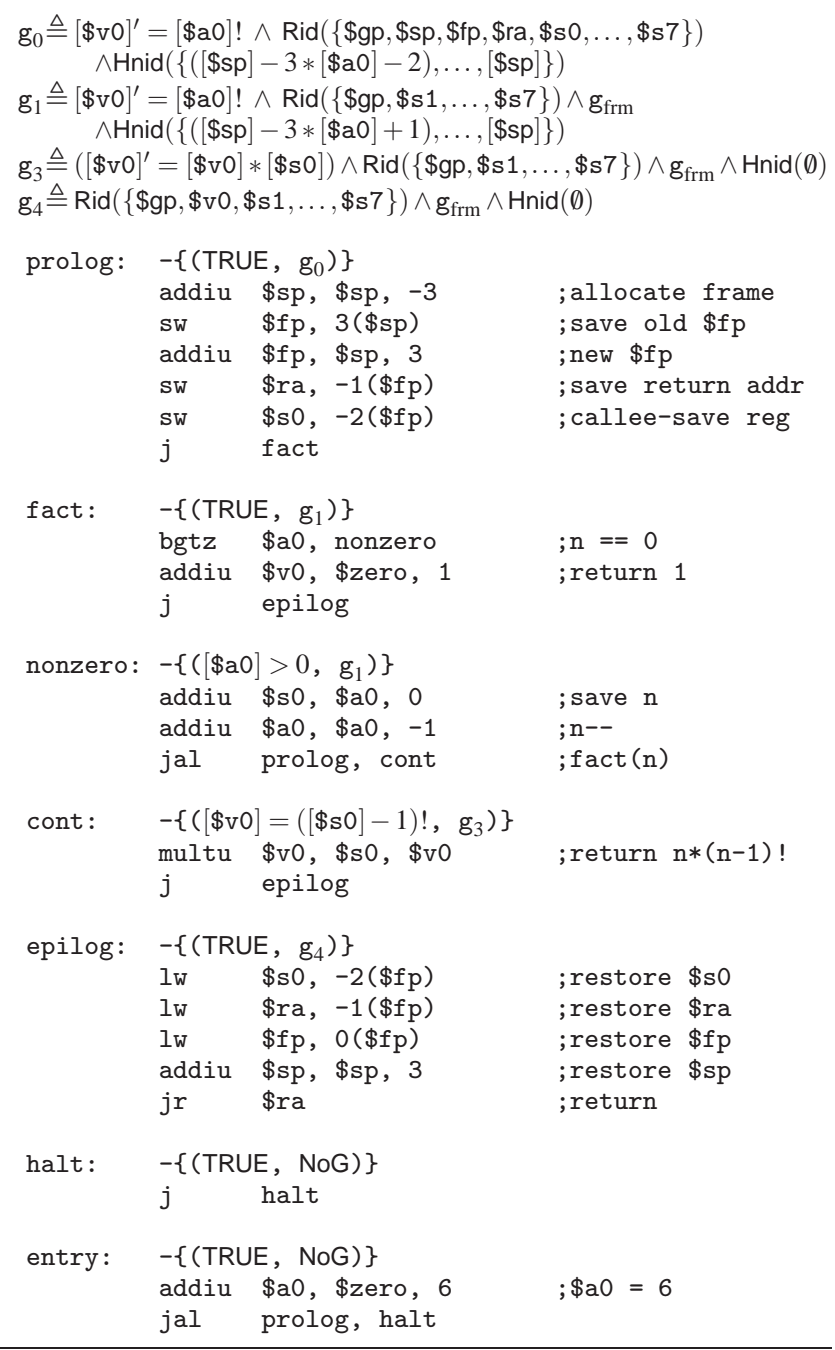

Figure 12. SCAP Factorial Example

direct jump. The precondition $\mathrm{p}_{0}$ requires that stack variable be initialized to 1 and not be allocated on the unused stack space. The guarantee $\mathrm{g}_{0}$ is similar to the one for the first version.

Malicious functions cannot be called. It is also interesting to see how malicious functions are rejected in SCAP. The following code shows a malicious function which disguises a function call of the virus code as a return (the more deceptive $\mathrm{x} 86$ version is "push virus; ret").

ld_vir: $-\{(p, g)\}$

addiu \$ra, \$zero, virus ; fake the ret addr

jr \$ra ; disguised func. call

The function ld_vir can be verified in SCAP with a proper specification of $(\mathrm{p}, \mathrm{g})$ (e.g., (TRUE, $\lambda \mathbb{S}, \mathbb{S}^{\prime}$.True)), because the SCAP RET rule does not check the return address in \$ra. However, SCAP will reject any code trying to call ld_vir, because the g cannot satisfy the premises of the CALL rule.

\section{Generalizations of SCAP}

The methodology for SCAP scales well to multi-return function calls and weak continuations. In this section, we will generalize the SCAP system in two steps. By a simple relaxation of the CALL rule, we get system SCAP-I to support function calls with multiple return addresses (with the restriction that a function must return to its immediate caller). We can use SCAP-I to certify the stack- 


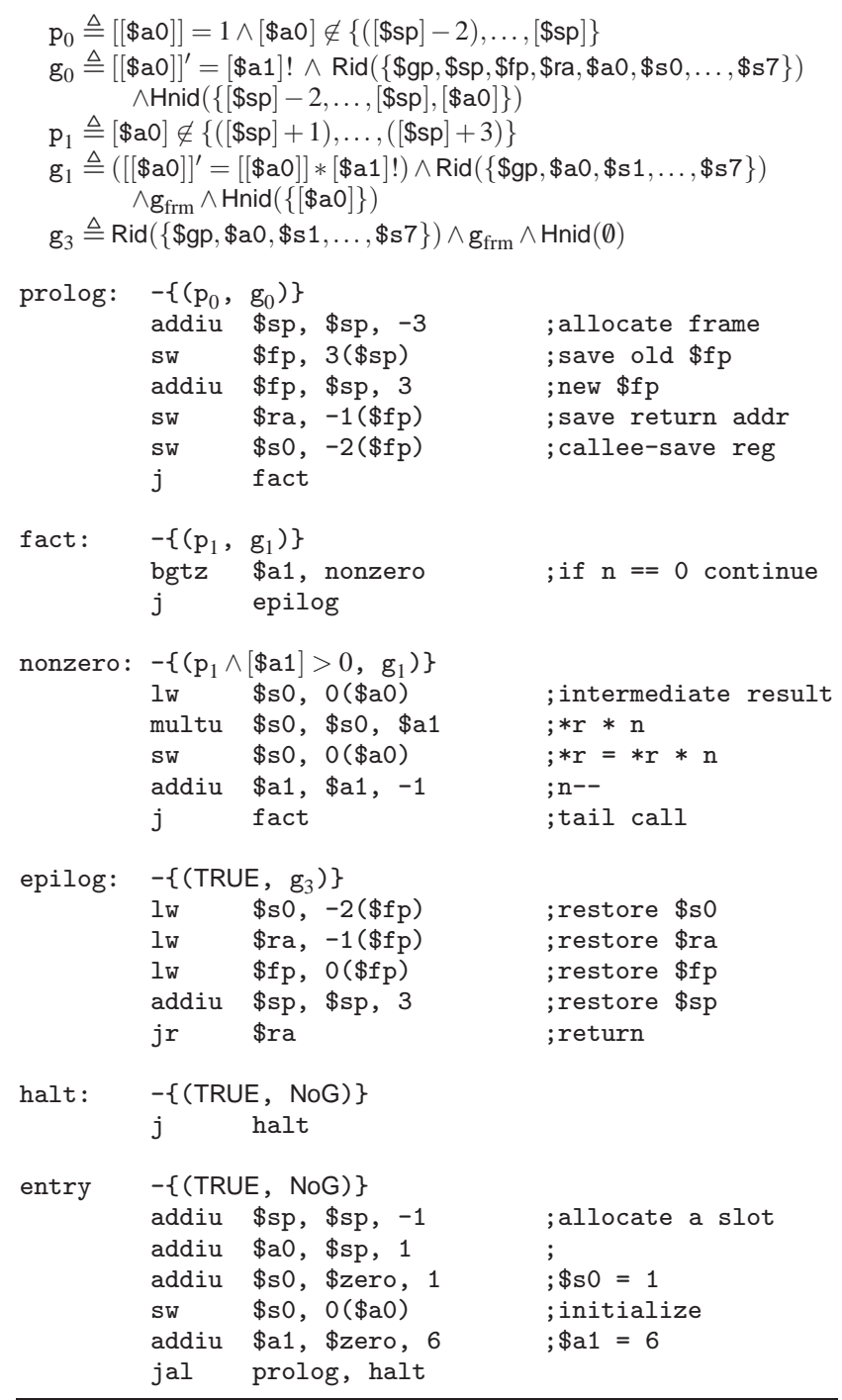

Figure 13. SCAP Implementation of Tail Recursion

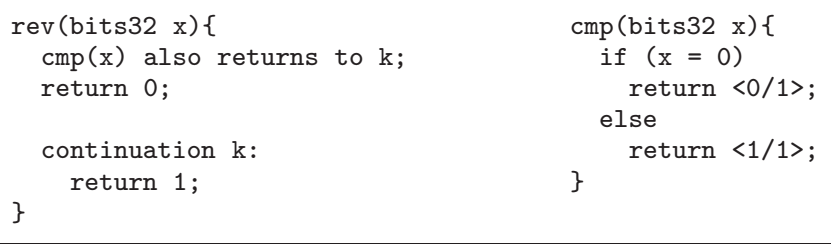

Figure 14. C-- Code with Multi-Return Address

unwinding-based implementation for exceptions. We then combine the relaxed call rule with the support for tail function call and get a more general system, namely SCAP-II. SCAP-II can certify weak continuations, setjmp/longjmp and the full-blown MRLC [32].

\subsection{SCAP-I}

In SCAP, a function call is a jal $f, f_{\text {ret }}$ instruction (equivalent to addiu $\$$ ra, $\left.\$ z e r o, f_{r e t} ; j f\right)$. The callee can only return to $f_{r e t}$, forced by the constraint $\forall \mathbb{S}, \mathbb{S}^{\prime} . \mathrm{g}^{\prime} \mathbb{S} \mathbb{S}^{\prime} \rightarrow \mathbb{S} \cdot \mathbb{R}(\$ \mathrm{ra})=\mathbb{S}^{\prime} . \mathbb{R}(\$ \mathrm{ra})$ in the CALL rule. To allow the callee to return to multiple locations, we simply remove that constraint. Also, since we no longer force a single return address, there is no need to set \$ra at the call site,

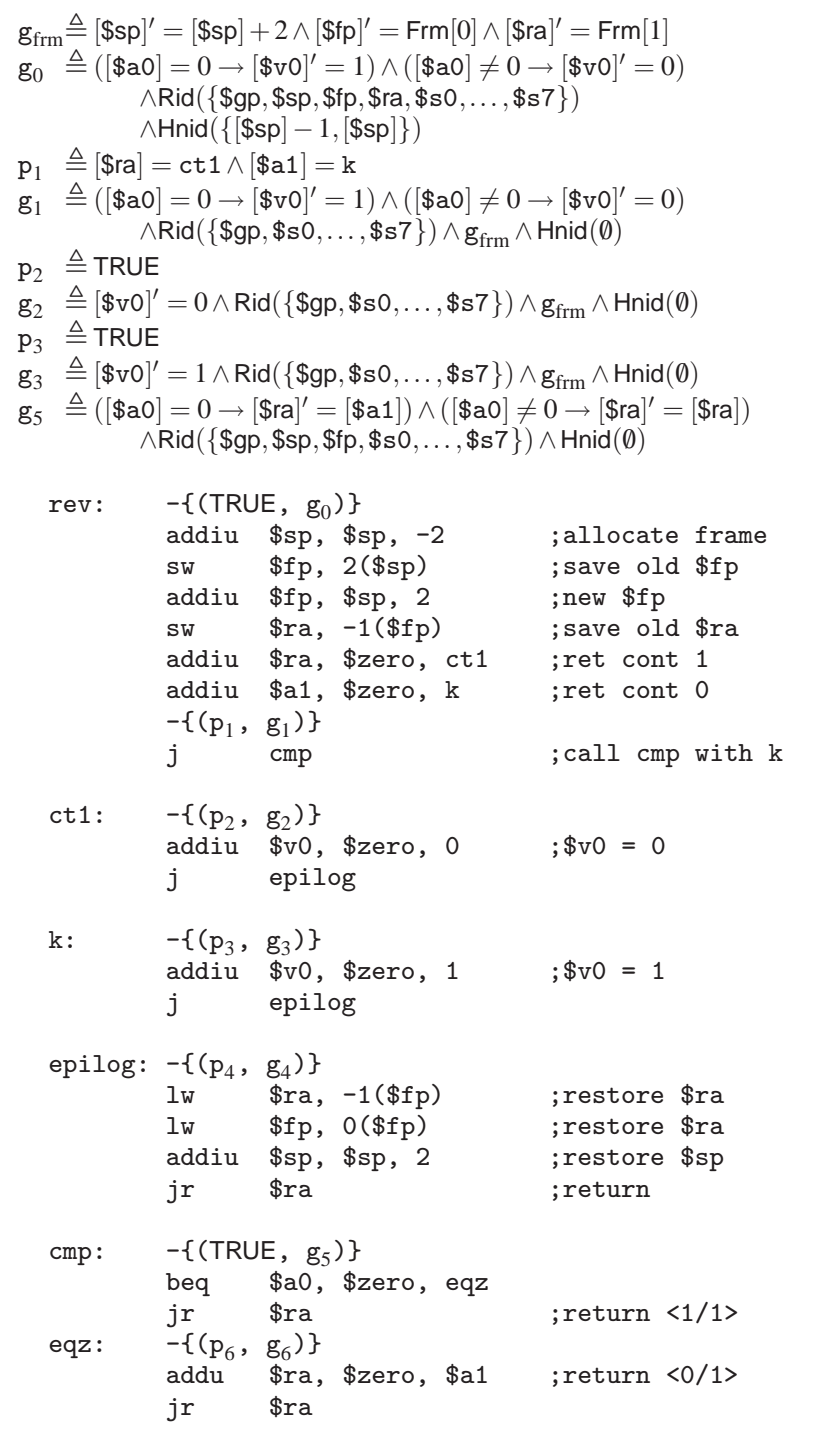

Figure 15. Example for Multi-Return Function Call

reducing the calling instruction to $\mathrm{j} f$. The resulting rule becomes

$$
\begin{aligned}
& \forall \mathbb{S} . \mathrm{p} S \rightarrow \mathrm{p}^{\prime} \mathbb{S} \quad \mathrm{f} \in \operatorname{dom}\left(\Psi_{\mathrm{L}}\right) \quad\left(\mathrm{p}^{\prime}, \mathrm{g}^{\prime}\right)=\Psi_{\mathrm{L}}(\mathrm{f}) \\
& \forall \mathbb{S}, \mathbb{S}^{\prime} \cdot \mathrm{p} \mathbb{S} \rightarrow \mathrm{g}^{\prime} \mathbb{S} \mathbb{S}^{\prime} \rightarrow \\
& \mathbb{S}^{\prime} \cdot \mathbb{R}(\$ r a) \in \operatorname{dom}\left(\Psi_{\mathrm{L}}\right) \wedge \mathrm{p}^{\prime \prime} \mathbb{S}^{\prime} \wedge\left(\forall \mathbb{S}^{\prime \prime} \cdot \mathrm{g}^{\prime \prime} \mathbb{S}^{\prime} \mathbb{S}^{\prime \prime} \rightarrow \mathrm{g} \mathbb{S} \mathbb{S}^{\prime \prime}\right) \\
& \text { where }\left(\mathrm{p}^{\prime \prime}, \mathrm{g}^{\prime \prime}\right)=\Psi_{\mathrm{L}}\left(\mathbb{S}^{\prime} \cdot \mathbb{R}(\$ \mathrm{ra})\right) \\
& \vdash\left\{\langle\llbracket(\mathrm{p}, \mathrm{g}) \rrbracket\rangle \Psi_{\mathrm{L}}\right\} \mathrm{j} f
\end{aligned}
$$

This rules does not specify how the return address is going to be passed into the function. Instead, we only require that \$ra contain a code pointer specified in $\Psi_{\mathrm{L}}$ at the return state $\mathbb{S}^{\prime}$, which is provable based on the knowledge of $\mathrm{p}$ and $\mathrm{g}^{\prime}$. This allows SCAP-I to certify any convention for multi-return function call.

The CALL-I rule is also a lemma provable from the $J$ rule of CAP0, using the same interpretation as the one for SCAP. The rest of SCAP-I inference rules are the same with those in SCAP. For instance, we can also use the T-CALL rule when we use " $\mathrm{f}$ " to make a tail call.

SCAP-I can certify the compiled C-- code with stack unwinding. C-- uses the primitive "return $\langle\mathrm{n} / \mathrm{m}\rangle$ " to allow a function to return to the $\mathrm{n}^{\text {th }}$ of $\mathrm{m}$ return continuations defined in the caller. A normal return is written as "return $<\mathrm{m} / \mathrm{m}\rangle$ ", while $\mathrm{n}$ being less 


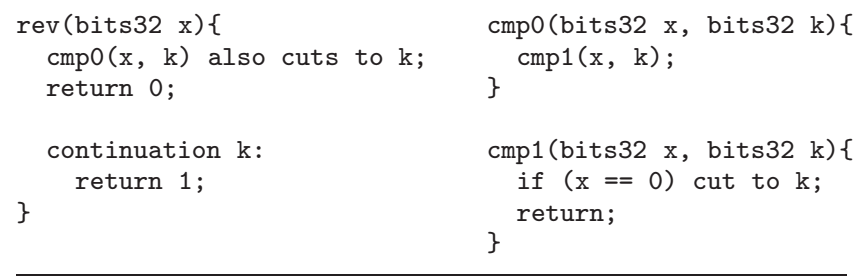

Figure 16. C-- Code with Weak-Continuations

than m means an "abnormal" return. Correspondingly, at the call cite, the caller put the annotation such as "also returns to $\mathrm{k} 0$, $\mathrm{k} 1$ ", where continuations $\mathrm{k} 0$ and $\mathrm{k} 1$ are defined in the same function as the call site.

In Figure 14 we show a simple C-- program which returns 1 if the argument is 0 and returns 0 otherwise. We illustrate in Figure 15 how to use SCAP-I to certify the compiled code. The precondition of the rev function is simply set to TRUE, while $\mathrm{g}_{0}$ specifies the relationship between the argument $[\$ \mathrm{a} 0]$ and the return value $[\$ \mathrm{v} 0]$, and the preservation of callee save registers and memory except for the space for the stack frame. The precondition for the cmp function is TRUE, and the guarantee $g_{5}$ says that the function returns to different addresses under different conditions.

At the point where cmp is called, we need to specify in the precondition $p_{1}$ that both return addresses are valid code labels (i.e., ct1 and $\mathrm{k})$. The guarantee $\mathrm{g}_{1}$ specifies the behavior of the remaining code under two different conditions, while $\left(\mathrm{p}_{2}, \mathrm{~g}_{2}\right)$ and $\left(\mathrm{p}_{3}, \mathrm{~g}_{3}\right)$ specify the two different return continuations. Interested readers can check that the specifications satisfy the constraint enforced by the CALL-I rule. Specifications for other code blocks are straightforward and are omitted here.

\subsection{SCAP-II for Weak Continuations}

The weak continuation construct in C-- allows a function to return to any activation on the control stack. Since we use the guarantee $\mathrm{g}$ to represent the behavior of a function, we need to understand what happens to the intermediate activations on the stack that are "skipped": are their g's discarded or fulfilled?

In SCAP-II, we enforce that the callee must fulfill the remaining behavior of its caller before it can "skip" its caller and return to an activation deeper on the control stack. From the caller's point of view, it made a tail call to the callee.

$$
\begin{aligned}
& \forall \mathbb{S} \text {. } \mathrm{p} \mathbb{S} \rightarrow \mathrm{p}^{\prime} \mathbb{S} \quad \mathrm{f} \in \operatorname{dom}\left(\Psi_{\mathrm{L}}\right) \quad\left(\mathrm{p}^{\prime}, \mathrm{g}^{\prime}\right)=\Psi_{\mathrm{L}}(\mathrm{f}) \\
& \forall \mathbb{S}, \mathbb{S}^{\prime} . \mathrm{p} \mathbb{S} \rightarrow \mathrm{g}^{\prime} \mathbb{S} \mathbb{S}^{\prime} \rightarrow \\
& \left(\mathrm{g} \mathbb{S} \mathbb{S}^{\prime} \vee\right. \\
& \left.\mathbb{S}^{\prime} . \mathbb{R}(\$ \mathrm{ra}) \in \operatorname{dom}\left(\Psi_{\mathrm{L}}\right) \wedge \mathrm{p}^{\prime \prime} \mathbb{S}^{\prime} \wedge\left(\forall \mathbb{S}^{\prime \prime} \cdot \mathrm{g}^{\prime \prime} \mathbb{S}^{\prime} \mathbb{S}^{\prime \prime} \rightarrow \mathrm{g} \mathbb{S} \mathbb{S}^{\prime \prime}\right)\right) \\
& \text { where }\left(\mathrm{p}^{\prime \prime}, \mathrm{g}^{\prime \prime}\right)=\Psi_{\mathrm{L}}\left(\mathbb{S}^{\prime} \cdot \mathbb{R}(\$ \mathrm{ra})\right) \\
& \vdash\left\{\langle\llbracket(\mathrm{p}, \mathrm{g}) \rrbracket\rangle_{\Psi_{\mathrm{L}}}\right\} \mathrm{j} f
\end{aligned}
$$

In the CALL-II rule, we further relax the second premise of the CALL-I rule and provide an option of either returning to the return point of the caller or satisfying the caller's remaining $g$ and therefore being able to return to the caller's caller. This requirement automatically forms arbitrary length chains that allow the return to go arbitrarily far in the stack. Also notice that the CALL-II rule is simply a combination of the CALL-I rule and the T-CALL in SCAP for tail call.

We also relax SCAP's definition of "well-formed stack" and allow dismissal of multiple stack frames at the return point. Using the new predicate WFST' defined in Figure 18 in the interpretation function for $(\mathrm{p}, \mathrm{g})$, we can derive the CALL-II rule as a lemma. The rest of SCAP-II inference rules are the same with those in SCAP. When a function jumps to a weak continuation, we use the same rule as the RET rule in SCAP, as shown below. Here we use a new name JWC (jump to weak continuations) to show that \$ra contains a

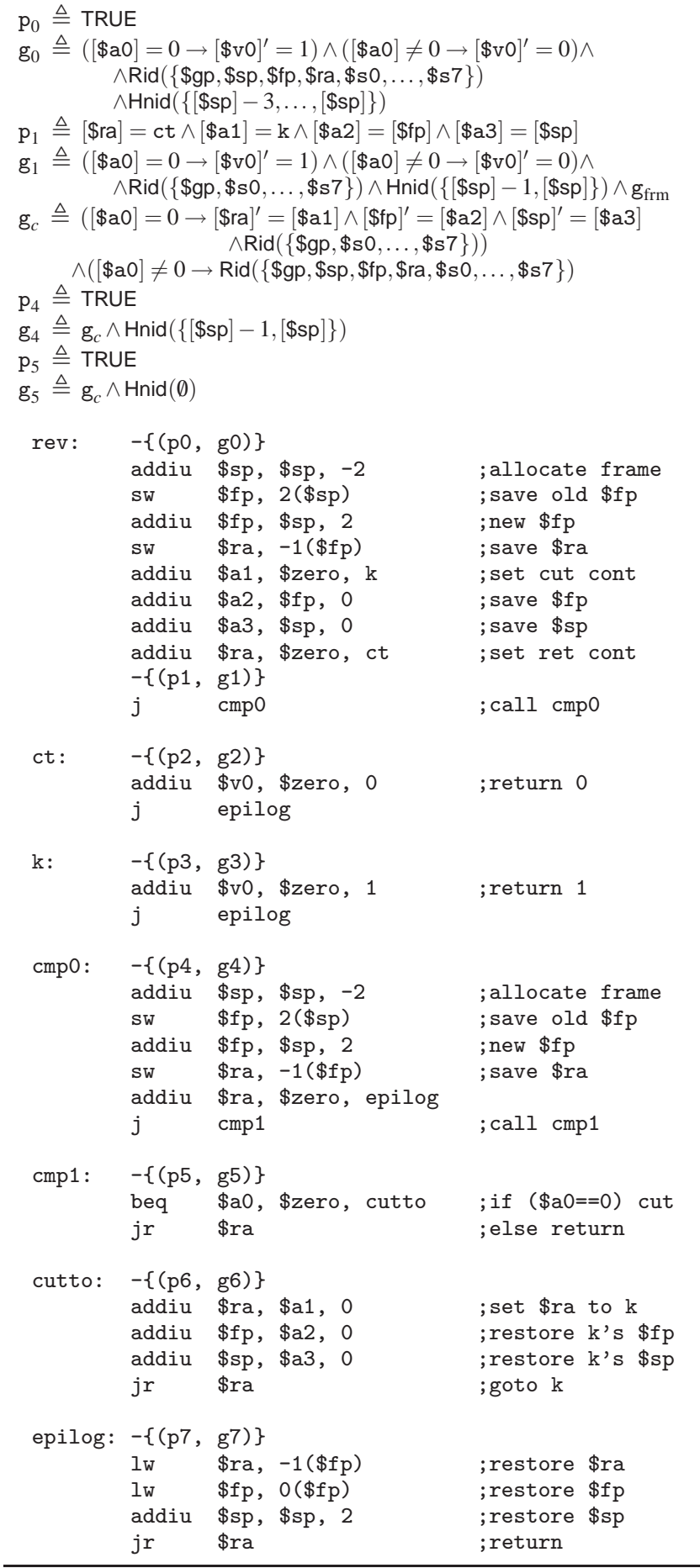

Figure 17. Example for Weak Continuation

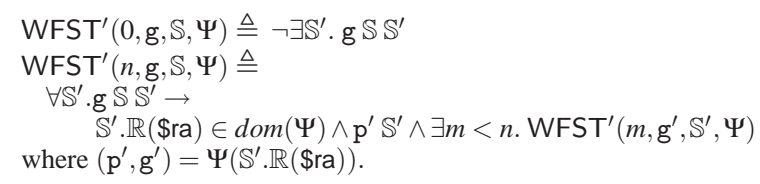

$\llbracket(\mathrm{p}, \mathrm{g}) \rrbracket \triangleq \lambda \Psi \cdot \lambda \mathrm{S} . \mathrm{p} \mathbb{S} \wedge \exists n \cdot \mathrm{WFST}^{\prime}(n, \mathrm{~g}, \mathbb{S}, \Psi)$

Figure 18. The Interpretation Function for SCAP-II 
weak continuation pointer instead of the return address in the caller.

$$
\frac{\forall \mathbb{S} . p \mathbb{S} \rightarrow \mathrm{g} \mathbb{S} \mathbb{S}}{\vdash\left\{\langle\llbracket(\mathrm{p}, \mathrm{g}) \rrbracket\rangle_{\Psi}\right\} \mathrm{jr} \$ \mathrm{ra}}
$$

Shivers and Fisher use the "super tail recursive" function call to implement their MRLC, which is essentially multi-return function call with stack cutting. The implementation of MRLC can be certified using SCAP-II.

In Figure 16, we show a C-- program using weak continuations. The behavior of the function rev is similar to the one shown in Figure 14. If the argument is 0 , the function cmp1 may skip over its caller cmp0 and cut to the stack of rev.

Figure 17 shows the compiled code and specifications. To simplify the presentation, we pass the weak continuation $\mathrm{k}$ (which contains the return code pointer, the frame pointer and the stack pointer) via registers $\$ a 1-\$ a 3$. The specification $\left(\mathrm{p}_{0}, \mathrm{~g}_{0}\right)$ for $\mathrm{rev}$ is very similar to the one shown in Figure 15. The specification at the call site of $\mathrm{cmp0}$ is $\left(\mathrm{p}_{1}, \mathrm{~g}_{1}\right)$. Specifications for functions cmp0 and cmp1 are given as $\left(\mathrm{p}_{4}, \mathrm{~g}_{4}\right)$ and $\left(\mathrm{p}_{5}, \mathrm{~g}_{5}\right)$, respectively. Notice that two different conditions are considered in $\mathrm{g}_{4}$ and $\mathrm{g}_{5}$, i.e., the condition under which that the functions return normally and the condition under which the functions cut the stack. Specifications for other code blocks are omitted.

\subsection{Example: setjmp/longjmp}

setjmp and longjmp are two functions in the $\mathrm{C}$ library that are used to perform non-local jumps. They are used as follows: a setjmp is called to save the current state of the program into a data structure (i.e., jmp_buf). That state contains the current stack pointer, all callee-save registers, the code pointer to the next instruction, and everything else prescribed by the architecture. Then when called with such a structure, longjmp restores every part of the saved state, and then jumps to the stored code pointer.

These functions in $\mathrm{C}$ are not considered safe. setjmp does not save closures, and thus the behavior of longjmp is undefined if the function calling the corresponding setjmp has returned. The control flow abstraction provided by setjmp/longjmp is very similar to weak continuations and can be reasoned using SCAP-II.

The code in Figure 19 shows a simple implementation of setjmp/longjmp functions and their specifications. Here we borrow the separation logic [31] notation, where $\{l \mapsto n\}$ means the memory cell at address $l$ contains value $n$, while $P * Q$ specifies two parts of memory which have disjoint domains and satisfy $P$ and $Q$ respectively. As shown in [40], separation logic primitives can be encoded in Coq and embedded in general predicates.

The precondition $\mathrm{p}_{0}$ of setjmp simply requires that the argument $\$ \mathrm{a} 0$ point to a jmp_buf. It guarantees $\left(\mathrm{g}_{0}\right)$ that the return value is 0 ; values of callee save registers, return code pointers and some other registers are not changed and they are saved in the jmp_buf; and data heap except the jmp_buf is not changed.

Precondition $\mathrm{p}_{1}$ for long jmp is similar to $\mathrm{p}_{0}$, with extra requirement that the second argument $\$ a 1$, which will be the return value, cannot be 0 . The guarantee $g_{1}$ says the function returns $\$ a 1$, recovers register values saved in jmp_buf (including return code pointers and stack pointers), and does not change any part of the memory.

In Figure 20 we use a simple $\mathrm{C}$ program to illustrate the use of setjmp/longjmp. The code has the same behavior with the one shown in Figure 16, except that here we make a non-local jump by using the setjmp/longjmp instead of a weak continuation.

Based on our specification of setjmp/longjmp, the compiled code of the $\mathrm{C}$ program can be certified using SCAP-II. The assembly code and specifications are presented in Figures 21 and 22. Here we reuse some macros defined previously in Figures 19 and 11.

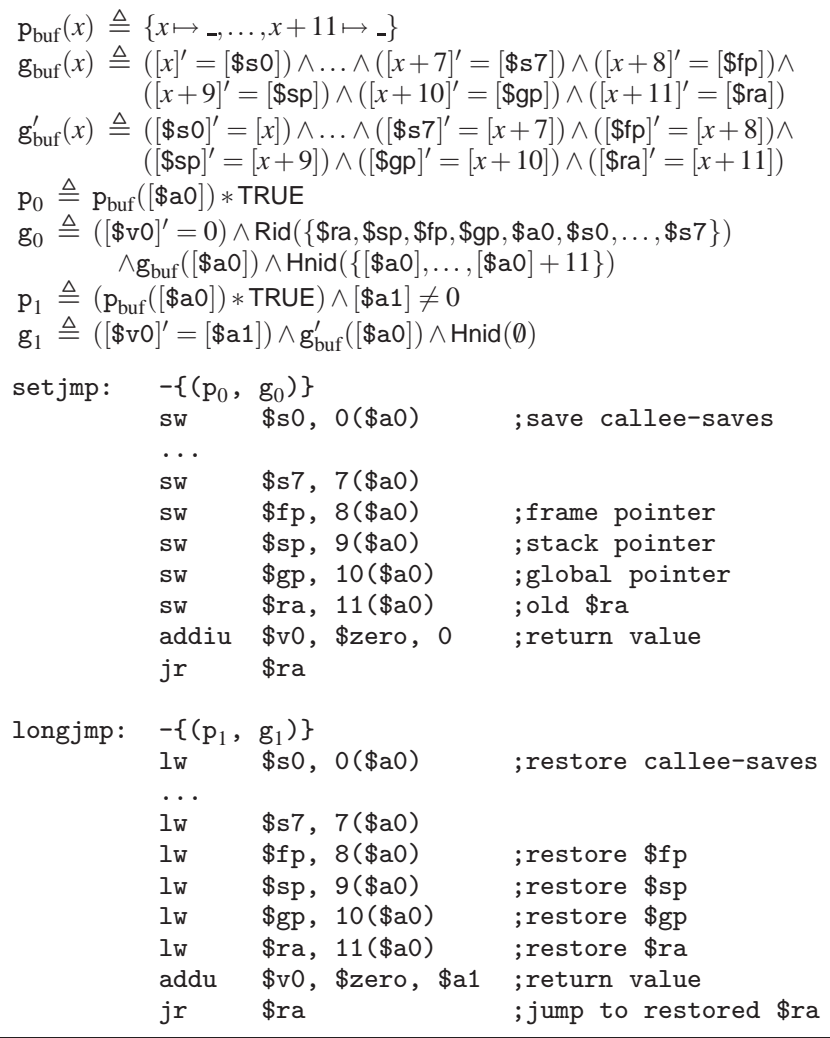

Figure 19. Implementation for setjmp/longjmp

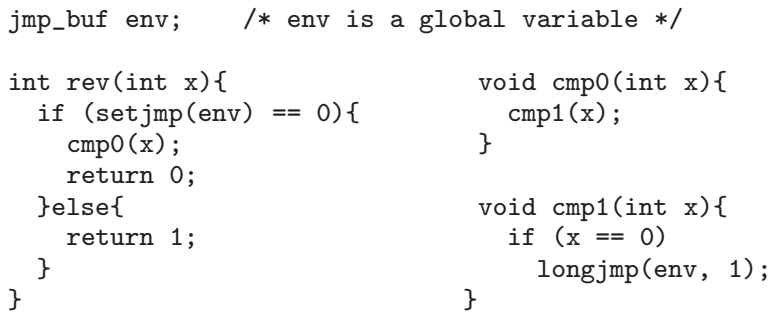

Figure 20. C Program Using setjmp/longjmp

The precondition $\mathrm{p}_{0}$ for function rev requires that env point to a block of memory for the jmp_buf, and that there be disjoint memory space for stack frames; while the guarantee $\mathrm{g}_{0}$ is similar to the one shown in Figure 17. Specifications for function cmp0 and cmp1 are similar to the ones given in Figure 17 too. However, it is a little tricky to specify the code labeled by ct1, which may be executed twice: the first time after the return from setjmp and the second time after the return from longjmp. We need to consider both cases in the specification $\left(\mathrm{p}_{1}, \mathrm{~g}_{1}\right)$.

\section{Exceptions}

Figure 23 shows a higher-level program with exception. The "try" block (line 3-6) encloses the code that may raise exceptions, while the "on error" block (line 9-12) defines an exception handler. If an exception is raised within the try block, the remaining code of of the block is skipped and the control jumps to line 9. Otherwise code following the block (line 7,8) is executed and the function returns. Note that if an exception is raised outside of the try block (e.g., from line 7-8 or 9-12), it will be handled by the closest higherlevel handler (not shown in the code snippet). Raising an exception is similar to a return, except this return does not go to the previous function, but rather to the closest exception handler. 


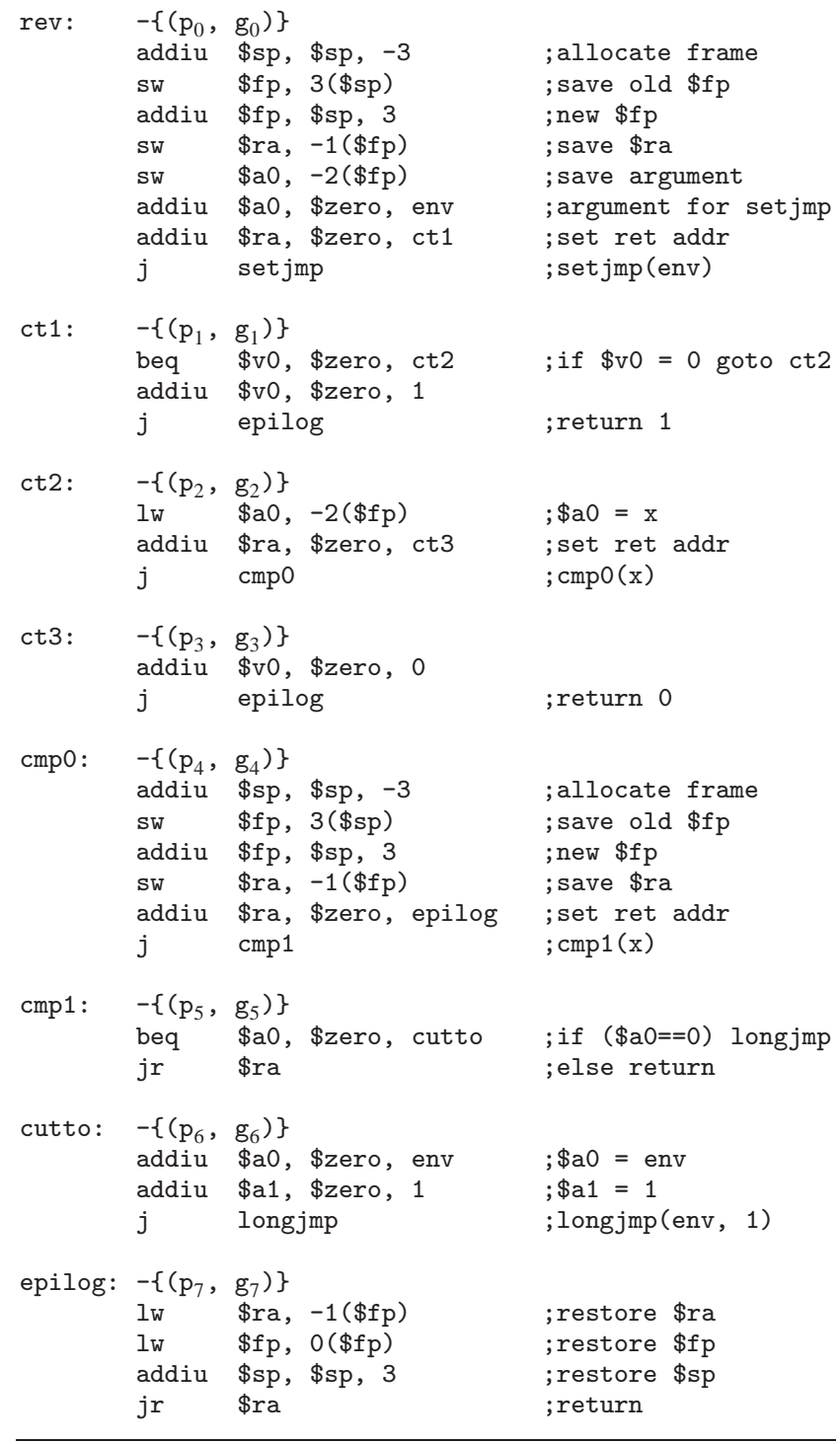

Figure 21. TM Code Using setjmp/longjmp

There are two stack-based implementation methods for exceptions [30]. One, called stack unrolling or stack unwinding, relies on the fact that each function has an implicit exception handler, which restores callee-saved registers from the stack and re-raises the exception. This method requires no extra operations upon entering a try block, as all the necessary state will be preserved by future function calls. However, upon raising an exception, the program will execute each implicit exception handler until the proper one is reached, meaning that this is a slow operation. The second method, stack cutting, requires that all callee-saves are stored upon entering a try block. Then, when an exception is raised, we can simply cut the stack to the appropriate point, and restore all the callee-saves to resume computation from the handler.

The SCAP-I system presented in Section 5 supports reasoning about stack unwinding. SCAP-II supports general weak continuation, therefore it can be used for both stack unwinding and stack cutting. However, with the emphasis on generality, the abstraction is too low level for these two systems to be used conveniently. For instance, in a function that has two possible return points, one would have to give a guarantee containing a disjunction to show that only under normal conditions the code will return to first re-

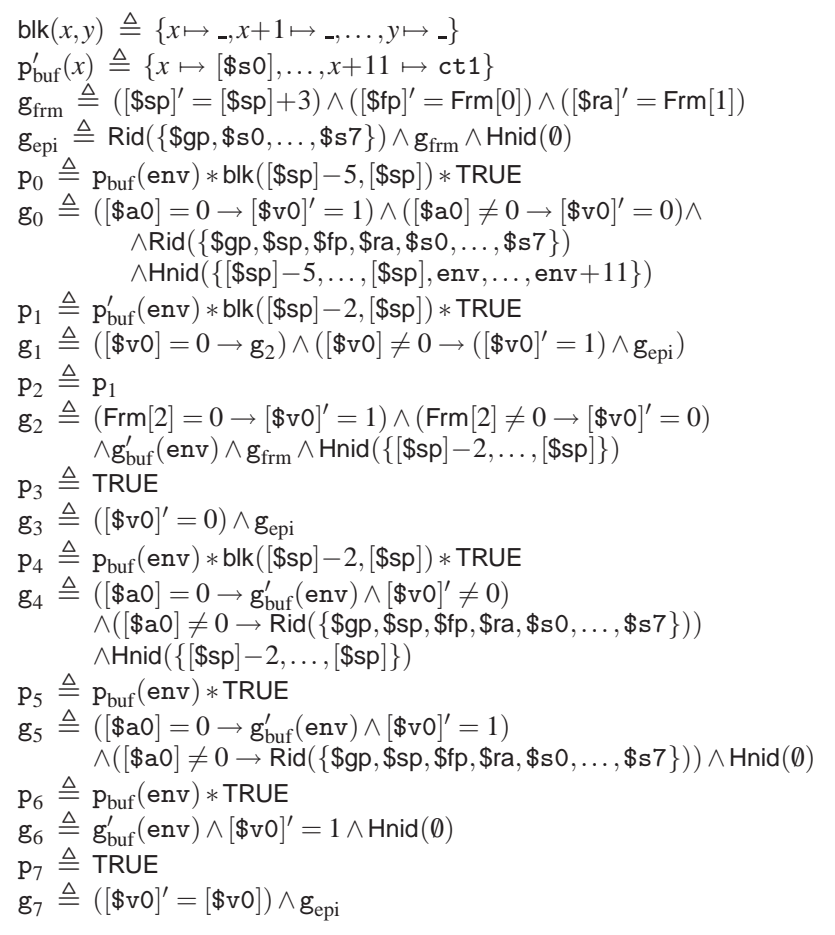

Figure 22. Specifications for Code in Figure 21

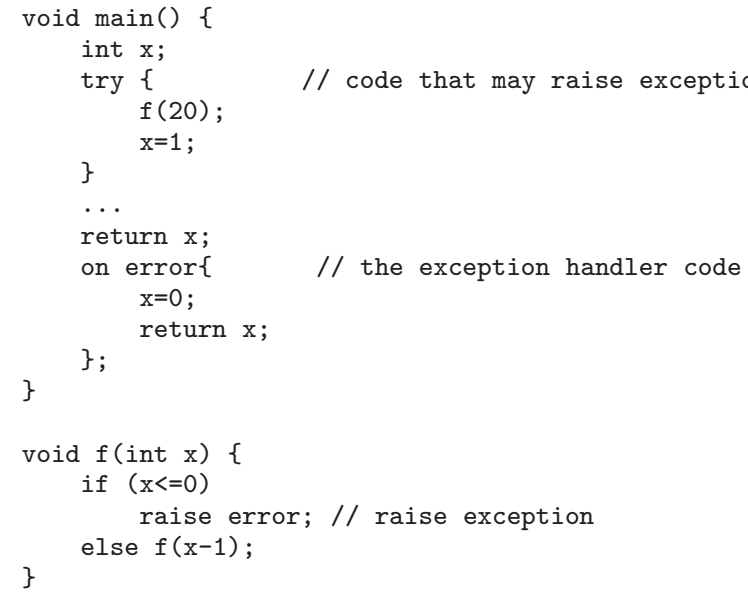

Figure 23. Higher-Level Pseudo Code with Exceptions

turn point, and only under exceptional conditions it will return to the second point. In this section, we propose two higher-level systems customized to handle stack unwinding and stack cutting respectively. Both of these systems provide guarantees specific to reasoning about exceptions.

\subsection{Exception Handling with Stack Unwinding}

In this section, we show a specification system that can support reasoning about exceptions compiled using stack unwinding, which we refer to as EUCAP. The system is embeddable in CAP0, and closely follows ideas used in defining SCAP.

\subsubsection{EUCAP Inference Rules}

Figure 24 shows the model of exception handling using stack unwinding. One should immediately observe that the try and call are not separate concepts. Both of them are represented by "call", which always sets a new exception handler. Thus in the diagram 


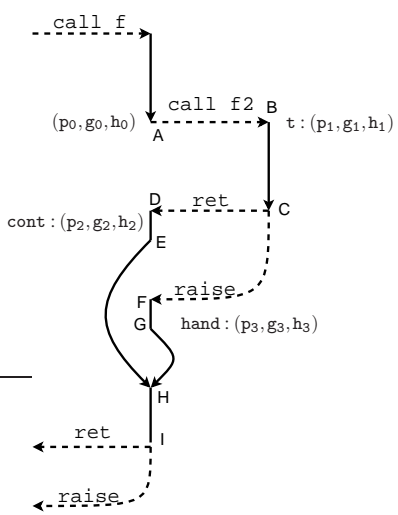

Figure 24. The Model for Call/Raise/Return in EUCAP

$$
\begin{aligned}
& \forall \mathbb{S} . \mathrm{p} \mathbb{S} \rightarrow \mathrm{p}^{\prime} \mathbb{S} \quad \mathrm{f} \in \operatorname{dom}\left(\Psi_{\mathrm{L}}\right) \quad\left(\mathrm{p}^{\prime}, \mathrm{g}^{\prime}, \mathrm{h}^{\prime}\right)=\Psi_{\mathrm{L}}(\mathrm{f}) \\
& \forall \mathbb{S} . p \mathbb{S} \rightarrow \mathbb{S} . \mathbb{R}(\$ r a) \in \operatorname{dom}\left(\Psi_{\mathrm{L}}\right) \wedge \\
& \forall \mathbb{S}^{\prime} \cdot \mathrm{g}^{\prime} \mathbb{S} \mathbb{S}^{\prime} \rightarrow \\
& \mathrm{p}^{\prime \prime} \mathbb{S}^{\prime} \wedge \forall \mathbb{S}^{\prime \prime} .\left(\mathrm{g}^{\prime \prime} \mathbb{S}^{\prime} \mathbb{S}^{\prime \prime} \rightarrow \mathrm{g} \mathbb{S} \mathbb{S}^{\prime \prime}\right) \wedge\left(\mathrm{h}^{\prime \prime} \mathbb{S}^{\prime} \mathbb{S}^{\prime \prime} \rightarrow \mathrm{h} \mathbb{S} \mathbb{S}^{\prime \prime}\right) \\
& \text { where }\left(\mathrm{p}^{\prime \prime}, \mathrm{g}^{\prime \prime}, \mathrm{h}^{\prime \prime}\right)=\Psi_{\mathrm{L}}(\mathbb{S} . \mathbb{R}(\$ \mathrm{ra})) \\
& \forall \mathbb{S} . \mathrm{p} \mathbb{S} \rightarrow \mathbb{S} . \mathbb{R}(\$ r h) \in \operatorname{dom}\left(\Psi_{\mathrm{L}}\right) \wedge \\
& \forall \mathbb{S}^{\prime} \cdot \mathrm{h}^{\prime} \mathbb{S} \mathbb{S}^{\prime} \rightarrow \\
& \mathrm{p}^{\prime \prime \prime} \mathbb{S}^{\prime} \wedge \forall \mathbb{S}^{\prime \prime} .\left(\mathrm{g}^{\prime \prime \prime} \mathbb{S}^{\prime} \mathbb{S}^{\prime \prime} \rightarrow \mathrm{g} \mathbb{S} \mathbb{S}^{\prime \prime}\right) \wedge\left(\mathrm{h}^{\prime \prime \prime} \mathbb{S}^{\prime} \mathbb{S}^{\prime \prime} \rightarrow \mathrm{h} \mathbb{S} \mathbb{S}^{\prime \prime}\right) \\
& \text { where }\left(\mathrm{p}^{\prime \prime \prime}, \mathrm{g}^{\prime \prime \prime}, \mathrm{h}^{\prime \prime \prime}\right)=\Psi_{\mathrm{L}}(\mathbb{S} . \mathbb{R}(\$ \mathrm{rh})) \\
& \forall \mathbb{S}, \mathbb{S}^{\prime} .\left(\mathrm{g}^{\prime} \mathbb{S} \mathbb{S}^{\prime} \rightarrow \mathbb{S} \cdot \mathbb{R}(\$ \mathrm{ra})=\mathbb{S}^{\prime} . \mathbb{R}(\$ \mathrm{ra})\right) \\
& \wedge\left(\mathrm{h}^{\prime} \mathbb{S} \mathbb{S}^{\prime} \rightarrow \mathbb{S} \cdot \mathbb{R}(\$ \mathrm{rh})=\mathbb{S}^{\prime} \cdot \mathbb{R}(\$ \mathrm{rh})\right) \\
& \vdash\left\{\langle\llbracket(\mathrm{p}, \mathrm{g}, \mathrm{h}) \rrbracket\rangle_{\Psi_{\mathrm{L}}}\right\} \mathrm{j} f
\end{aligned}
$$

$$
\begin{gathered}
\frac{\forall \mathbb{S} . p \mathbb{S} \rightarrow \mathrm{g} \mathbb{S} \mathbb{S}}{\vdash\left\{\langle\llbracket(\mathrm{p}, \mathrm{g}, \mathrm{h}) \rrbracket\rangle_{\Psi_{\mathrm{L}}}\right\} \text { jr } \$ \mathrm{ra}} \\
\frac{\forall \mathbb{S} . \mathrm{p} \mathbb{S} \rightarrow \mathrm{h} \mathbb{S} \mathbb{S}}{\vdash\left\{\langle\llbracket(\mathrm{p}, \mathrm{g}, \mathrm{h}) \rrbracket\rangle_{\Psi_{\mathrm{L}}}\right\} \text { jr } \$ \mathrm{rh}}
\end{gathered}
$$

$$
\mathrm{c} \in\{\text { addu, addiu, Iw, subu, sw }\}
$$$$
\left.\left.\vdash\left\{\left\langle\llbracket\left(\mathrm{p}^{\prime}, \mathrm{g}^{\prime}, \mathrm{h}^{\prime}\right)\right]\right\rangle\right\rangle_{\mathrm{L}}\right\} \mathbb{I}
$$$$
\forall \mathbb{S} . p \mathbb{S} \rightarrow \mathrm{p}^{\prime}\left(\operatorname{Next}_{\mathrm{c}}(\mathbb{S})\right)
$$$$
\forall \mathbb{S}, \mathbb{S}^{\prime} \cdot \mathrm{p} \mathbb{S} \rightarrow\left(\mathrm{g}^{\prime} \operatorname{Next}_{\mathrm{c}}(\mathbb{S}) \mathbb{S}^{\prime} \rightarrow \mathrm{g} \mathbb{S}^{\prime}\right) \wedge\left(\mathrm{h}^{\prime} \operatorname{Next}_{\mathrm{c}}(\mathbb{S}) \mathbb{S}^{\prime} \rightarrow \mathrm{h} \mathbb{S} \mathbb{S}^{\prime}\right)
$$

$$
\vdash\left\{\left\langle[(\mathrm{p}, \mathrm{g}, \mathrm{h}) \rrbracket\rangle_{\Psi_{\mathrm{L}}}\right\} \mathrm{c} ; \mathbb{I}\right.
$$

(EU-SEQ)

\section{Figure 25. EUCAP Rules as CAP0 Lemmas}

above, one can think of call $\mathrm{f} 2$ as either a function call or a try block, which sets $\mathrm{D}$ as the return point and $\mathrm{F}$ as an exception handler. Thus when at point $\mathrm{C}$, an exception is raised, the execution jumps to point $\mathrm{F}$, and proceeds to execute from there. The code in the handler then may finish normally by returning, or may choose to re-raise the exception by issuing raise. That behavior is not any different from a regular return point, and that fact is represented in the diagram by a merging at point $\mathrm{H}$. One can think of point $\mathrm{H}$ as where the try block ends and regular execution resumes.

Given the above diagram, it is very easy to define exceptions. We define them simply as another return point, and to cleanly specify these return points separately, we extend SCAP's specification language by adding another predicate similar to $g$. Then the specification language becomes

$$
\begin{aligned}
& \text { (Assertion) p, } \mathrm{q}, \mathrm{r} \in \text { State } \rightarrow \text { Prop } \\
& \text { (Guarantee) } \mathrm{g}, \mathrm{h} \quad \in \text { State } \rightarrow \text { State } \rightarrow \text { Prop } \\
& \text { (CdSpec) } \quad \theta \quad::=(\mathrm{p}, \mathrm{g}, \mathrm{h})
\end{aligned}
$$

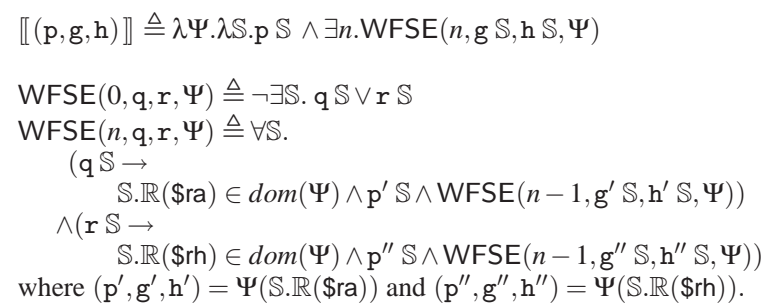

Figure 26. EUCAP Interpretation

The $\mathrm{h}$ guarantee specifies what a function does by the time an exception is raised. If a function can not raise an exception, then $\mathrm{h}$ can simply be set to $\forall \mathbb{S}, \mathbb{S}^{\prime}$.False. This means that this model is actually the same as multi-return function call (SCAP-I), with $g$ split into two pieces: one for regular return, another for exceptional return.

The rest of the system is just a correction for this split: a raise is simply a ret when $\mathrm{h}$ as opposed to $\mathrm{g}$ is met. The call needs to check that both predicates are adequate, and the well-formedness of the stack needs to be altered to allow both predicates to specify possible return points. The complete set of EUCAP rules is given in figure 25, and the interpretation function is given in figure 26.

Derivations of EUCAP rules as lemma in the CAP0 framework can be found in appendix B.

\subsubsection{Example}

In this section we show an example of how to use EUCAP to certify a program that implements exceptions using stack unwinding. The higher level program shown in figure 23 is compiled with stack unwinding implementation of exceptions to produce assembly code shown in figure 27.

Label main is an entry point into the program. Labels main-1 and main-2 are the normal and exceptional continuations for the program. Label $f$ is the entry point of function $f$, while $f$-raise and f-return are the code blocks responsible for raising an exception or returning from function $f$.

Specifications for each block of code are embedded in the code right next to the label they specify. To make specification more readable, we reuse the macros defined before in Figure 11 for common expressions.

In this example, all jumps to $f$ are EU-CALL instructions, which always set a new handler. This makes the try block around the call to $f$ be unnecessary, and thus the try block and the first call to function $f$ are merged. $x$ is set to 1 at the normal return point, but not at the exceptional return point, making that instruction work as though it is inside a try block, while it is actually outside of the call. Thus the $g$ for $f$-ret and $h$ for $f$-raise simply say that they restore the previous frame and then jump to their appropriate return point.

\subsection{Exception Handling with Stack Cutting}

In this section, we show a specification system that can support reasoning about exceptions compiled using stack cutting, which we refer to as ECAP. The system is also embeddable in CAP0, and closely follows ideas used in defining SCAP.

\subsubsection{ECAP Inference Rules}

Figure 28 shows multiple workflows through the code containing exceptions. Function $f$ does a try, which then calls $f 2$. If $f 2$ succeeds, it then issues a return into the remaining segment inside a try (cont). If the entire try block succeeds, then endtry jumps to cont2, which finishes the execution of function $f$. However, if an exception is raised inside the try block, including inside $f 2$, 


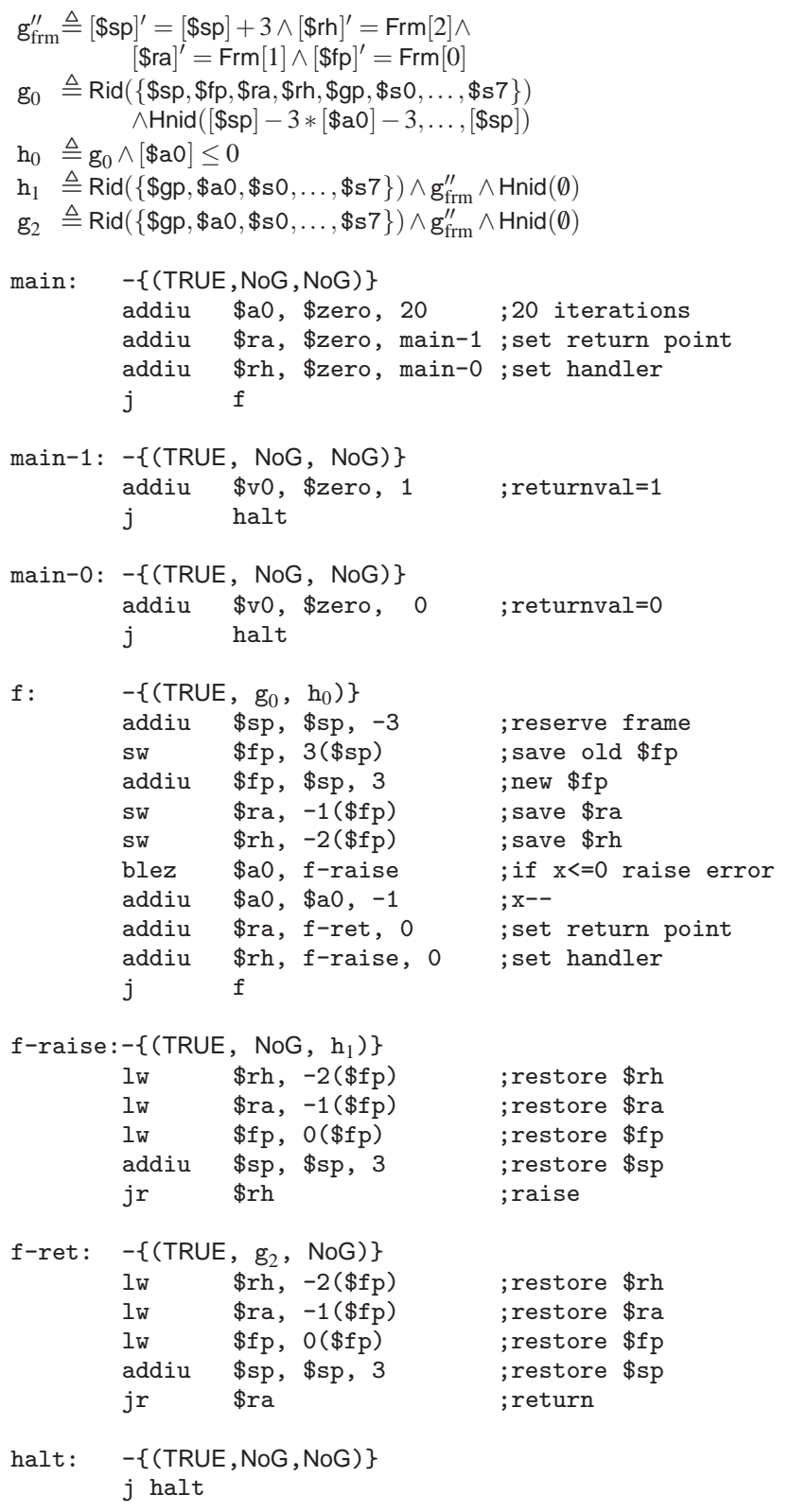

Figure 27. Example of Exceptions Compiled with Unwinding

the program jumps to the handler specified by the try block (hand). Then both execution paths from cont 2 and hand will eventually return to the same point, or raise an exception and return to the same handler.

To be able to certify code with exceptions implemented using stack cutting, we define ECAP, another instance of CAP0 inspired by SCAP. The code specification of ECAP are same as those in EUCAP:

$$
\begin{aligned}
& \text { (Assertion) } \mathrm{p}, \mathrm{q}, \mathrm{r} \in \text { State } \rightarrow \text { Prop } \\
& \text { (Guarantee) } \mathrm{g}, \mathrm{h} \in \text { State } \rightarrow \text { State } \rightarrow \text { Prop } \\
& \text { (CdSpec) } \quad \theta \quad::=(\mathrm{p}, \mathrm{g}, \mathrm{h})
\end{aligned}
$$

The purpose of $h$ is exactly the same as its purpose in EUCAP, namely to specify what needs to happen between the current point and the point where the exception can be raised.

To ensure correct behavior in all executions, we enforce the following invariants at the point of entering a try block:

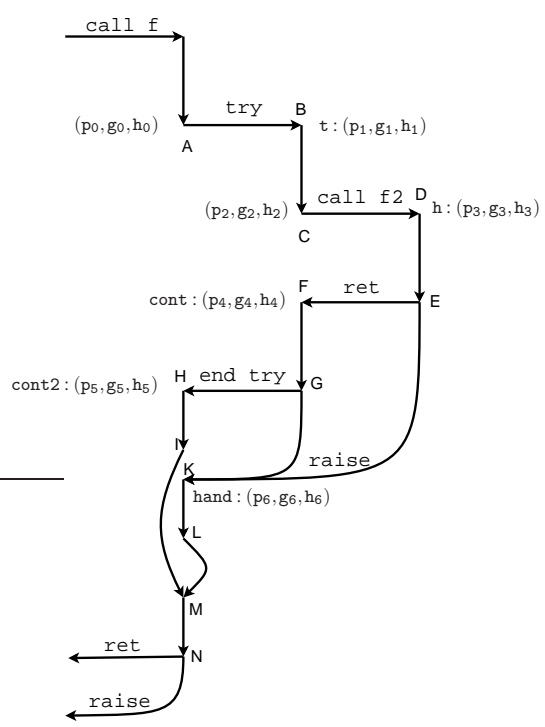

Figure 28. The Model for Try/Raise/Call/Return in ECAP

$$
\begin{aligned}
& \forall \mathbb{S} . \mathrm{p} \mathbb{S} \rightarrow \mathrm{p}^{\prime} \mathbb{S} \quad \mathrm{f} \in \operatorname{dom}\left(\Psi_{\mathrm{L}}\right) \quad\left(\mathrm{p}^{\prime}, \mathrm{g}^{\prime}, \mathrm{h}^{\prime}\right)=\Psi_{\mathrm{L}}(\mathrm{f}) \\
& \forall \mathbb{S} . \mathrm{p} \mathbb{S} \rightarrow \mathbb{S} . \mathbb{R}(\$ r a) \in \operatorname{dom}\left(\Psi_{\mathrm{L}}\right) \wedge \\
& \forall \mathbb{S}^{\prime} \cdot \mathrm{g}^{\prime} \mathbb{S} \mathbb{S}^{\prime} \rightarrow \\
& \mathrm{p}^{\prime \prime} \mathbb{S}^{\prime} \wedge \forall \mathbb{S}^{\prime \prime} .\left(\mathrm{g}^{\prime \prime} \mathbb{S}^{\prime} \mathbb{S}^{\prime \prime} \rightarrow \mathrm{g} \mathbb{S} \mathbb{S}^{\prime \prime}\right) \wedge\left(\mathrm{h}^{\prime \prime} \mathbb{S}^{\prime} \mathbb{S}^{\prime \prime} \rightarrow \mathrm{h} \mathbb{S} \mathbb{S}^{\prime \prime}\right) \\
& \text { where }\left(\mathrm{p}^{\prime \prime}, \mathrm{g}^{\prime \prime}, \mathrm{h}^{\prime \prime}\right)=\Psi_{\mathrm{L}}(\mathbb{S} \cdot \mathbb{R}(\$ \mathrm{ra})) \\
& \forall \mathbb{S}, \mathbb{S}^{\prime} . \mathrm{p} \mathbb{S} \rightarrow \mathrm{h}^{\prime} \mathbb{S} \mathbb{S}^{\prime} \rightarrow \mathrm{h} \mathbb{S} \mathbb{S}^{\prime} \quad \forall \mathbb{S}, \mathbb{S}^{\prime} \cdot \mathrm{g}^{\prime} \mathbb{S} \mathbb{S}^{\prime} \rightarrow \mathbb{S} . \mathbb{R}(\$ \mathrm{ra})=\mathbb{S}^{\prime} \cdot \mathbb{R}(\$ \mathrm{ra}) \\
& \vdash\left\{\langle\llbracket(\mathrm{p}, \mathrm{g}, \mathrm{h}) \rrbracket\rangle_{\Psi_{\mathrm{L}}}\right\} \mathrm{j} f
\end{aligned}
$$

$$
\begin{aligned}
& \forall \mathbb{S} . \mathrm{p} \mathbb{S} \rightarrow \mathrm{p}^{\prime} \mathbb{S} \quad \mathrm{S} \in \operatorname{dom}\left(\Psi_{\mathrm{L}}\right) \quad\left(\mathrm{p}^{\prime}, \mathrm{g}^{\prime}, \mathrm{h}^{\prime}\right)=\Psi_{\mathrm{L}}(\mathrm{f}) \\
& \forall \mathbb{S} . \mathrm{p} \mathbb{S} \rightarrow \mathbb{S} . \mathbb{R}(\$ \mathrm{ra}) \in \operatorname{dom}\left(\Psi_{\mathrm{L}}\right) \wedge \\
& \quad \forall \mathbb{S}^{\prime} . \mathrm{g}^{\prime} \mathbb{S} \mathbb{S}^{\prime} \rightarrow \\
& \quad \mathrm{p}^{\prime \prime} \mathbb{S}^{\prime} \wedge \forall \mathbb{S}^{\prime \prime} .\left(\mathrm{g}^{\prime \prime} \mathbb{S}^{\prime} \mathbb{S}^{\prime \prime} \rightarrow \mathrm{g} \mathbb{S} \mathbb{S}^{\prime \prime}\right) \wedge\left(\mathrm{h}^{\prime \prime} \mathbb{S}^{\prime} \mathbb{S}^{\prime \prime} \rightarrow \mathrm{h} \mathbb{S} \mathbb{S}^{\prime \prime}\right) \\
& \text { where }\left(\mathrm{p}^{\prime \prime}, \mathrm{g}^{\prime \prime}, \mathrm{h}^{\prime \prime}\right)=\Psi_{\mathrm{L}}(\mathbb{S} . \mathbb{R}(\$ \mathrm{ra}))
\end{aligned}
$$

$$
\begin{aligned}
& \forall \mathbb{S} . p \mathbb{S} \rightarrow \mathbb{S} . \mathbb{R}(\$ r h) \in \operatorname{dom}\left(\Psi_{\mathrm{L}}\right) \wedge \\
& \forall \mathbb{S}^{\prime} \cdot \mathrm{h}^{\prime} \mathbb{S} \mathbb{S}^{\prime} \rightarrow \\
& \mathrm{p}^{\prime \prime \prime} \mathbb{S}^{\prime} \wedge \forall \mathbb{S}^{\prime \prime} .\left(\mathrm{g}^{\prime \prime \prime} \mathbb{S}^{\prime} \mathbb{S}^{\prime \prime} \rightarrow \mathrm{g} \mathbb{S} \mathbb{S}^{\prime \prime}\right) \wedge\left(\mathrm{h}^{\prime \prime \prime} \mathbb{S}^{\prime} \mathbb{S}^{\prime \prime} \rightarrow \mathrm{h} \mathbb{S} \mathbb{S}^{\prime \prime}\right) \\
& \text { where }\left(\mathrm{p}^{\prime \prime \prime}, \mathrm{g}^{\prime \prime \prime}, \mathrm{h}^{\prime \prime \prime}\right)=\Psi_{\mathrm{L}}(\mathbb{S} \cdot \mathbb{R}(\$ \mathrm{rh}))
\end{aligned}
$$

$\forall \mathbb{S}, \mathbb{S}^{\prime} \cdot\left(\mathrm{g}^{\prime} \mathbb{S} \mathbb{S}^{\prime} \rightarrow \mathbb{S} \cdot \mathbb{R}(\$ \mathrm{ra})=\mathbb{S}^{\prime} \cdot \mathbb{R}(\$ \mathrm{ra})\right)$

$\wedge\left(\mathrm{h}^{\prime} \mathbb{S} \mathbb{S}^{\prime} \rightarrow \mathbb{S} . \mathbb{R}(\$ \mathrm{rh})=\mathbb{S}^{\prime} . \mathbb{R}(\$ \mathrm{rh})\right)$

$$
\vdash\left\{\left\langle\lfloor(\mathrm{p}, \mathrm{g}, \mathrm{h}) \rrbracket\rangle_{\Psi_{\mathrm{L}}}\right\} \mathrm{j} f\right.
$$

$$
\begin{gathered}
\frac{\forall \mathbb{S} . p \mathbb{S} \rightarrow \mathrm{g} \mathbb{S} S}{\vdash\left\{\langle\llbracket(\mathrm{p}, \mathrm{g}, \mathrm{h}) \rrbracket\rangle_{\Psi_{\mathrm{L}}}\right\} \text { jr } \$ \mathrm{ra}} \\
\frac{\forall \mathbb{S} . \mathrm{p} \mathbb{S} \rightarrow \mathrm{h} \mathbb{S} \mathbb{S}}{\vdash\left\{\langle\llbracket(\mathrm{p}, \mathrm{g}, \mathrm{h}) \rrbracket\rangle_{\Psi_{\mathrm{L}}}\right\} \mathrm{jr} \$ \mathrm{rh}}
\end{gathered}
$$

$$
\begin{aligned}
& \mathrm{c} \in\{\text { addu, addiu, } \mathrm{lw}, \mathrm{subu}, \mathrm{sw}\} \\
& \vdash\left\{\left\langle\llbracket\left(\mathrm{p}^{\prime}, \mathrm{g}^{\prime}, \mathrm{h}^{\prime}\right) \rrbracket\right\rangle_{\Psi_{\mathrm{L}}}\right\} \mathbb{I} \quad \forall \text { S.p } \mathbb{S} \rightarrow \mathrm{p}^{\prime}\left(\operatorname{Next}_{\mathrm{c}}(\mathbb{S})\right) \\
& \forall \mathbb{S}, \mathbb{S}^{\prime} . \mathrm{p} \mathbb{S} \rightarrow\left(\mathrm{g}^{\prime} \operatorname{Next}_{\mathrm{c}}(\mathbb{S}) \mathbb{S}^{\prime} \rightarrow \mathrm{g} \mathbb{S} \mathbb{S}^{\prime}\right) \wedge\left(\mathrm{h}^{\prime} \operatorname{Next}_{\mathrm{c}}(\mathbb{S}) \mathbb{S}^{\prime} \rightarrow \mathrm{h} \mathbb{S} \mathbb{S}^{\prime}\right) \\
& \vdash\left\{\langle\llbracket(\mathrm{p}, \mathrm{g}, \mathrm{h}) \rrbracket\rangle_{\Psi_{\mathrm{L}}}\right\} \mathrm{c} ; \mathbb{I}
\end{aligned}
$$

Figure 29. ECAP Rules as CAP0 Lemmas 
$\llbracket(\mathrm{p}, \mathrm{g}, \mathrm{h}) \rrbracket \triangleq \lambda \Psi . \lambda \mathbb{S} . \mathrm{p} \mathbb{S} \wedge \exists n . \operatorname{WFSC}(n, \mathrm{~g} \mathbb{S}, \mathrm{h} \mathbb{S}, \Psi)$

$$
\begin{aligned}
& \operatorname{WFSC}(0, \mathrm{q}, \mathrm{r}, \Psi) \triangleq \neg \exists \mathbb{S} . \mathrm{q} \mathbb{S} \vee r \mathbb{S} \\
& \operatorname{WFSC}(n, \mathrm{q}, r, \Psi) \triangleq \forall \mathbb{S} \text {. } \\
& (\mathrm{q} \mathbb{S} \rightarrow \\
& \wedge(r \mathbb{S} \rightarrow \\
& \left.\mathbb{S} . \mathbb{R}(\$ r a) \in \operatorname{dom}(\Psi) \wedge \mathrm{p}^{\prime} \mathbb{S} \wedge \operatorname{WFSC}\left(n-1, \mathrm{~g}^{\prime} \mathbb{S}, \mathrm{h}^{\prime} \mathbb{S}, \Psi\right)\right) \\
& \left.\mathbb{S} . \mathbb{R}(\$ \mathrm{rh}) \in \operatorname{dom}(\Psi) \wedge \mathrm{p}^{\prime \prime} \mathbb{S} \wedge \exists m<n . \operatorname{WFSC}\left(m, \mathrm{~g}^{\prime \prime} \mathbb{S}, \mathrm{h}^{\prime \prime} \mathbb{S}, \Psi\right)\right)
\end{aligned}
$$$$
\text { where }\left(\mathrm{p}^{\prime}, \mathrm{g}^{\prime}, \mathrm{h}^{\prime}\right)=\Psi(\mathbb{S} . \mathbb{R}(\$ \mathrm{ra})) \text { and }\left(\mathrm{p}^{\prime \prime}, \mathrm{g}^{\prime \prime}, \mathrm{h}^{\prime \prime}\right)=\Psi(\mathbb{S} . \mathbb{R}(\$ \mathrm{rh})) \text {. }
$$

Figure 30. ECAP Interpretation

- the precondition of the try block $t$ can be satisfied, i.e., $\forall \mathbb{S} . \mathrm{p}_{0} \mathbb{S} \rightarrow \mathrm{p}_{1} \mathbb{S}$ (note that entering a try block is done by $\mathrm{j}$ );

- after the try block finishes normally, $f$ can resume execution beyond the try block (point $\mathrm{H}$ ), and the normal behavior $\left(\mathrm{g}_{1}\right)$ of the try block (B-G) and the remaining code (H-I) satisfies the specification of $\mathrm{A}-\mathrm{I}$, i.e.,

$$
\begin{aligned}
& \forall \mathbb{S}, \mathbb{S}^{\prime} \cdot \mathrm{p}_{0} \mathbb{S} \rightarrow \mathrm{g}_{1} \mathbb{S} \mathbb{S}^{\prime} \rightarrow \\
& \mathrm{p}_{5} \mathbb{S}^{\prime} \wedge\left(\forall \mathbb{S}^{\prime \prime} \cdot \mathrm{g}_{5} \mathbb{S}^{\prime} \mathbb{S}^{\prime \prime} \rightarrow \mathrm{g}_{0} \mathbb{S} \mathbb{S}^{\prime \prime}\right) \wedge\left(\forall \mathbb{S}^{\prime \prime} \cdot \mathrm{h}_{5} \mathbb{S}^{\prime} \mathbb{S}^{\prime \prime} \rightarrow \mathrm{h}_{5} \mathbb{S} \mathbb{S}^{\prime \prime}\right)
\end{aligned}
$$

- after the try block finishes exceptionally, $f$ can resume execution starting at the exception handler (point $\mathrm{K}$ ), and the exceptional behavior $\left(\mathrm{h}_{1}\right)$ of the try block and the exception handler (K-L) satisfies the specification of A-I, i.e.,

$$
\begin{aligned}
& \forall \mathbb{S}, \mathbb{S}^{\prime} \cdot \mathrm{p}_{0} \mathbb{S} \rightarrow \mathrm{h}_{1} \mathbb{S} \mathbb{S}^{\prime} \rightarrow \\
& \mathrm{p}_{6} \mathbb{S}^{\prime} \wedge\left(\forall \mathbb{S}^{\prime \prime} \cdot \mathrm{g}_{6} \mathbb{S}^{\prime} \mathbb{S}^{\prime \prime} \rightarrow \mathrm{g}_{0} \mathbb{S} \mathbb{S}^{\prime \prime}\right) \wedge\left(\forall \mathbb{S}^{\prime \prime} \cdot \mathrm{h}_{6} \mathbb{S}^{\prime} \mathbb{S}^{\prime \prime} \rightarrow \mathrm{h}_{0} \mathbb{S} \mathbb{S}^{\prime \prime}\right)
\end{aligned}
$$

The above conditions (in a generalized form) are enforced by the E-TRY rule shown in figure 29.

The E-CALL rule is an extension of the CALL rule of SCAP with following differences:

- adds an exceptional dual of the continuation's guarantee satisfying the function's guarantee, i.e.,

$\forall \mathbb{S}, \mathbb{S}^{\prime} \cdot \mathrm{p}_{2} \mathbb{S} \rightarrow \mathrm{g}_{3} \mathbb{S} \mathbb{S}^{\prime} \rightarrow\left(\forall \mathbb{S}^{\prime \prime} . \mathrm{h}_{4} \mathbb{S}^{\prime} \mathbb{S}^{\prime \prime} \rightarrow \mathrm{h}_{2} \mathbb{S} \mathbb{S}^{\prime \prime}\right)$.

- the function's exceptional behavior must satisfy the caller's exceptional behavior, i.e.,

$\forall \mathbb{S}, \mathbb{S}^{\prime} \cdot \mathrm{p}_{2} \mathbb{S} \rightarrow \mathrm{h}_{3} \mathbb{S} \mathbb{S}^{\prime} \rightarrow \mathrm{h}_{2} \mathbb{S} \mathbb{S}^{\prime}$.

Returning from the function call or a try block has the same invariant as the SCAP RET rule, i.e., the state in which a return is issued satisfies the predicate $g$. Raising an exception is a dual of return, having the same invariant using $\mathrm{h}$ predicate. Well-formedness of an instruction sequence is similar to the SCAP-SEQ rule, except it also ensures the chaining of the predicate $h$.

\subsubsection{ECAP in the CAPO Framework}

Just like SCAP, ECAP rules are derivable as lemmas of the CAP0 framework, using the definitions in figures 29 and 30. The interpretation of $(p, g, h)$ is similar to that of $(p, g)$ in SCAP. The new "well-formed stack" predicate (WFSC) is SCAP's WFST modified to deal with $\mathrm{h}$ and the non-linear stack workflow of exceptions. Namely, it adds an additional requirement that at the end of the exception handler there is a well formed stack of smaller depth (not just $n-1$, as in SCAP).

Like WFST, WFSC is recursive, well-founded, and is independent of the stack layout, supporting different calling conventions and exception handling methods.

WFSC predicate uses the \$rh register. However, the TM does not have such a register. This is done for simplicity, and should be interpreted as any specific memory which can be used to pass a second return point. In our examples, we take the liberty of making \$rh refer to the otherwise unused register $\mathrm{k} 1$, as well as using $\mathrm{k} 0$ as a pointer to where the handler keeps information necessary to restore the stack frame.

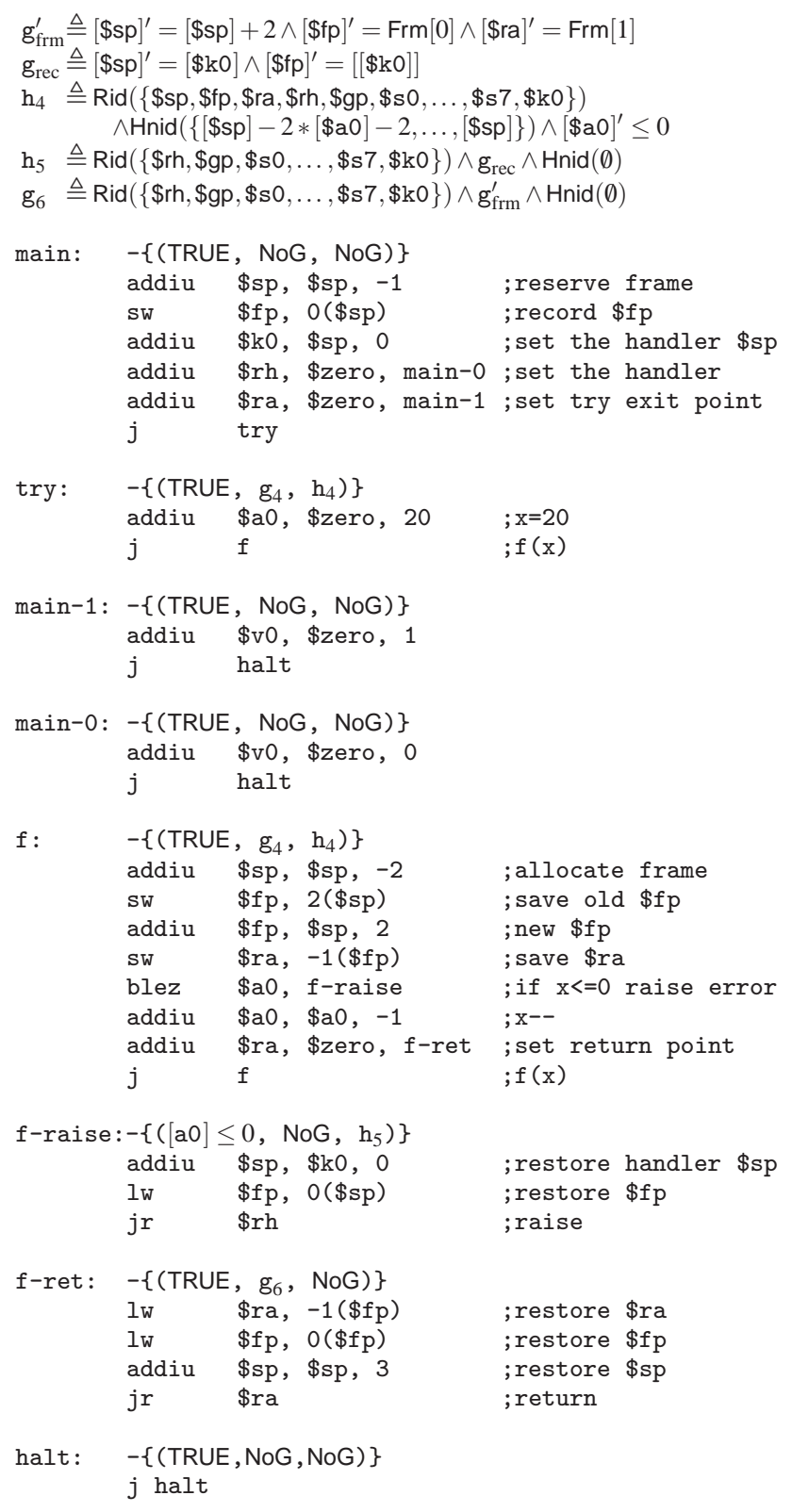

Figure 31. Example of Exceptions Compiled with Cutting

The derivation of ECAP rules as lemmas in the CAP0 framework can be found in appendix C.

\subsubsection{Example}

In this section we show how ECAP can be used to support exceptions using stack cutting.

The higher-level program given in Figure 23 is compiled using stack cutting, resuling in assembly code in figure 31 .

Specifications of code heaps are embedded in the code. We reuse macros defined before in Figures 11.

In this example, upon entering the try block, the callee-saves are saved on the stack, with \$rh remembering the handler's label and $\$ \mathrm{k} 0$ remembering the stack pointer of the main function. \$rh and $\$ \mathrm{k} 0$ are preserved through the calls to $\mathrm{f}$. When the exception is raised, the stack is recovered through $\$ \mathrm{k} 0$ and convention defined by $g_{\text {rec }}$. 


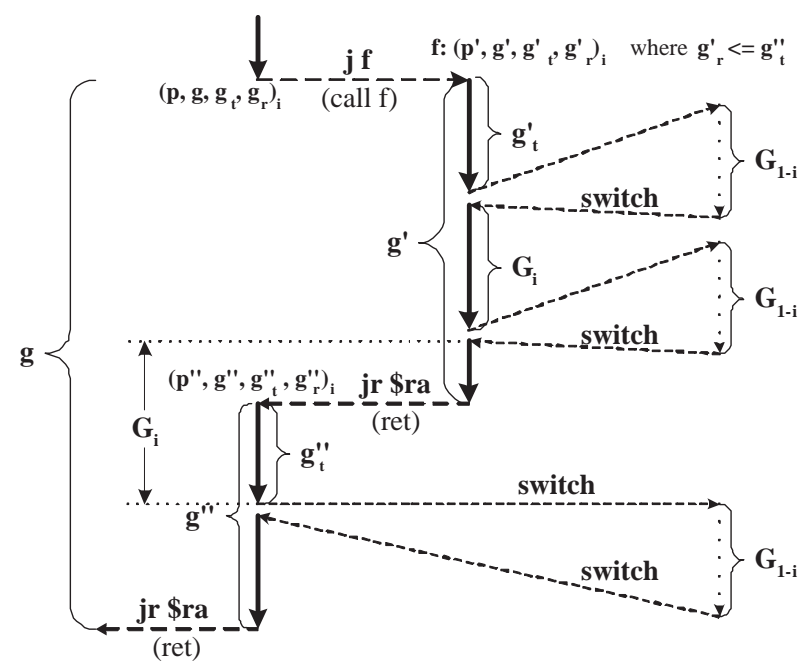

Figure 34. Model for Coroutines with Function Calls

may switch to another coroutine in the middle. It is harder to specify the behavior of functions.

In SCAP-CR, we instantiate the code specification $\theta$ in CAP0 as follows:

$$
\begin{aligned}
& \text { (Assertion) } \quad \mathrm{p} \quad \in \quad \text { State } \rightarrow \text { Prop } \\
& \text { (Guarantee) } \mathrm{g}, \mathrm{g}_{t}, \mathrm{~g}_{r} \in \text { State } \rightarrow \text { State } \rightarrow \text { Prop } \\
& (\text { CdSpec }) \quad \theta \quad::=\left(\mathrm{p}, \mathrm{g}, \mathrm{g}_{t}, \mathrm{~g}_{r}\right)_{i} \text { where }(0 \leq i \leq 1)
\end{aligned}
$$

As in SCAP, the function specification in SCAP-CR contains the specification $\mathrm{p}$ of the expected input and the behavior $\mathrm{g}$ of the function. Since a switch may occur within a function, we use $g_{t}$ as in CAP-CR to specify the code segment from the current point to the next switch point, as shown in Figure 34. Also, because the return point and the switch point may not match, we use an extra guarantee $g_{r}$ to specify the remaining state transition the current coroutine needs to make between the return point and the next switch point. Intuitively, $\mathrm{g}_{r}$ tells the caller of the current function what the caller needs to do after the function returns so that it can fulfill the guaranteed behavior before switching to another coroutine. ${ }^{1}$ The switch operation is implemented in the same way shown in Section 7.1. For each coroutine, we also need a global guarantee $\mathbb{G}_{i}$ which captures the invariant of the code segments between any two consecutive switch points.

As shown in Figure 34, we need to enforce the following constraints for the function call in SCAP-CR.

- the behavior $\mathrm{g}_{t}^{\prime}$ satisfies the caller's guaranteed behavior $\mathrm{g}_{t}$ from the calling point to the next switch point;

- when the callee returns, the caller's behavior $\mathrm{g}_{t}^{\prime \prime}$ from the return point to the next switch point satisfies the callee's expectation $\mathrm{g}_{r}^{\prime}$; and

- the constraints for return code pointers and function behaviors, as enforced in the CALL rule of SCAP-I.

\footnotetext{
${ }^{1}$ We may not need $\mathrm{g}_{r}$ if we require that the global guarantee $\mathbb{G}$ be a transitive relation, i.e., $\forall \mathbb{S}, \mathbb{S}^{\prime}, \mathbb{S}^{\prime \prime} . \mathbb{G} \mathbb{S} \mathbb{S}^{\prime} \wedge \mathbb{G S}^{\prime} \mathbb{S}^{\prime \prime} \rightarrow \mathbb{G} \mathbb{S} \mathbb{S}^{\prime \prime}$. Although reasonable in a non-deterministic concurrent setting, this constraint on $\mathbb{G}$ is too restrictive for coroutines. We decide to present SCAP-CR in the most general setting and use an extra $g_{r}$ to link the caller and callee.
}

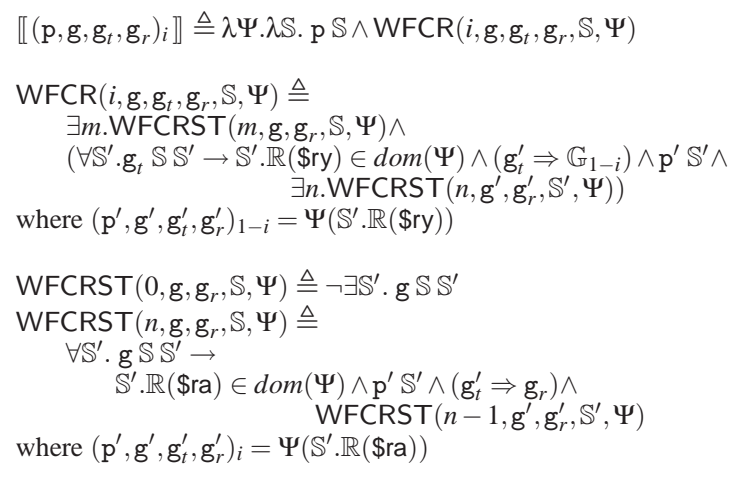

Figure 35. The Interpretation Function for SCAP-CR

These constraints are reflected in the following CR-CALL rule.

$$
\begin{aligned}
& \left(\mathrm{p}^{\prime}, \mathrm{g}^{\prime}, \mathrm{g}_{t}^{\prime}, \mathrm{g}_{r}^{\prime}\right)_{i}=\Psi_{\mathrm{L}}(\mathrm{f}) \\
& \forall \mathbb{S} \text {. p } \mathbb{S} \rightarrow \mathrm{p}^{\prime} \mathbb{S} \\
& \forall \mathbb{S}, \mathbb{S}^{\prime} . \mathrm{p} \mathbb{S} \rightarrow \mathrm{g}^{\prime} \mathbb{S} \mathbb{S}^{\prime} \rightarrow \\
& \forall \mathbb{S}, \mathbb{S}^{\prime} . \mathrm{p} \mathbb{S} \rightarrow \mathrm{g}_{t}^{\prime} \mathbb{S} \mathbb{S}^{\prime} \rightarrow \mathrm{g}_{t} \mathbb{S} \mathbb{S}^{\prime} \\
& \left(\mathbb{S}^{\prime} . \mathbb{R}(\$ \mathrm{ra}) \in \operatorname{dom}\left(\Psi_{\mathrm{L}}\right) \wedge \mathrm{p}^{\prime \prime} \mathbb{S}^{\prime} \wedge\right. \\
& \left.\left(\forall \mathbb{S}^{\prime \prime} \cdot \mathrm{g}^{\prime \prime} \mathbb{S}^{\prime} \mathbb{S}^{\prime \prime} \rightarrow \mathrm{g} \mathbb{S} \mathbb{S}^{\prime \prime}\right) \wedge\left(\forall \mathbb{S}^{\prime \prime} \cdot \mathrm{g}_{t}^{\prime \prime} \mathbb{S}^{\prime} \mathbb{S}^{\prime \prime} \rightarrow \mathrm{g}_{r}^{\prime} \mathbb{S}^{\prime} \mathbb{S}^{\prime \prime}\right)\right) \\
& \text { where }\left(\mathrm{p}^{\prime \prime}, \mathrm{g}^{\prime \prime}, \mathrm{g}_{t}^{\prime \prime}, \mathrm{g}_{r}\right)_{i}=\Psi_{\mathrm{L}}\left(\mathbb{S}^{\prime} \cdot \mathbb{R}(\text { (\$ra })\right) \\
& \vdash\left\{\left\langle\llbracket\left(\mathrm{p}, \mathrm{g}, \mathrm{g}_{t}, \mathrm{~g}_{r}\right)_{i} \rrbracket\right\rangle_{\Psi_{\mathrm{L}}}\right\} \mathrm{j} f
\end{aligned}
$$

The return rule CR-RET is similar to the RET rule in SCAP, except that we also need to ensure that the expected caller's behavior $\mathrm{g}_{r}$ from the return point to the next switch point satisfies the guaranteed behavior $\mathrm{g}_{t}$.

$$
\frac{\forall \mathbb{S} . \mathrm{p} \mathbb{S} \rightarrow \mathrm{g} \mathbb{S} \mathbb{S} \quad \forall \mathbb{S}, \mathbb{S}^{\prime} \cdot \mathrm{p} \mathbb{S} \rightarrow \mathrm{g}_{r} \mathbb{S} \mathbb{S}^{\prime} \rightarrow \mathrm{g}_{t} \mathbb{S} \mathbb{S}^{\prime}}{\vdash\left\{\left\langle\llbracket\left(\mathrm{p}, \mathrm{g}, \mathrm{g}_{t}, \mathrm{~g}_{r}\right)_{i} \rrbracket\right\rangle_{\Psi_{\mathrm{L}}}\right\} \mathrm{jr} \text { \%ra }}
$$

(CR-RET)

The CR-SWITCH rule is similar to the SWITCH rule in CAP-CR, but we also need to enforce that the guaranteed behavior of the function is satisfied, i.e., $\mathbb{G}_{1-i} \mathbb{S} \mathbb{S}^{\prime} \rightarrow \mathrm{g}^{\prime} \mathbb{S}^{\prime} \mathbb{S}^{\prime \prime} \rightarrow \mathrm{g} \mathbb{S} \mathbb{S}^{\prime \prime}$.

$$
\begin{aligned}
& \forall \mathbb{S} . \mathrm{p} \mathbb{S} \rightarrow \mathrm{g}_{t} \mathbb{S} \mathbb{S} \\
& \forall \mathbb{S} \text {. } \mathrm{p} \mathbb{S} \rightarrow \\
& \left(\mathbb{S} . \mathbb{R}(\$ r x) \in \operatorname{dom}\left(\Psi_{\mathrm{L}}\right) \wedge\left(\mathrm{g}_{t}^{\prime} \Rightarrow \mathbb{G}_{i}\right)\right. \\
& \left(\forall \mathbb{S}^{\prime} \cdot \mathbb{G}_{1-i} \mathbb{S} \mathbb{S}^{\prime} \rightarrow\right. \\
& \left.\left.\mathbb{S}^{\prime} \cdot \mathbb{R}(\$ r y)=\mathbb{S} \cdot \mathbb{R}(\$ r x) \wedge \mathrm{p}^{\prime} \mathbb{S}^{\prime} \wedge\left(\forall \mathbb{S}^{\prime \prime} \cdot \mathrm{g}^{\prime} \mathbb{S}^{\prime} \mathbb{S}^{\prime \prime} \rightarrow \mathrm{g} \mathbb{S} \mathbb{S}^{\prime \prime}\right)\right)\right) \\
& \text { where }\left(\mathrm{p}^{\prime}, \mathrm{g}^{\prime}, \mathrm{g}_{t}^{\prime}, \mathrm{g}_{r}\right)_{i}=\Psi_{\mathrm{L}}(\mathbb{S} . \mathbb{R}(\$ \mathrm{rx})) \\
& \vdash\left\{\left\langle\left\lfloor\left(\mathrm{p}, \mathrm{g}, \mathrm{g}_{t}, \mathrm{~g}_{r}\right)_{i} \rrbracket\right\rangle_{\Psi_{\mathrm{L}}}\right\} \text { jr } \$\right. \text { ry }
\end{aligned}
$$

The following CR-SEQ rule is straightforward, which is simply a combination of the SEQ rules in SCAP and CAP-CR.

$$
\begin{aligned}
& \begin{array}{l}
\vdash\left\{\left\langle\llbracket\left(\mathrm{p}^{\prime}, \mathrm{g}^{\prime}, \mathrm{g}_{t}^{\prime}, \mathrm{g}_{r}\right)_{i} \rrbracket\right\rangle \Psi_{\mathrm{L}}\right\} \mathbb{I} \quad \forall \mathbb{S} . \mathrm{p} \mathbb{S} \rightarrow \mathrm{p}^{\prime}\left(\operatorname{Next}_{\mathrm{c}}(\mathbb{S})\right) \\
\forall \mathbb{S}, \mathbb{S}^{\prime} \cdot \mathrm{p} \mathbb{S} \rightarrow \\
\left(\mathrm{g}^{\prime}\left(\operatorname{Next}_{\mathrm{c}}(\mathbb{S})\right) \mathbb{S}^{\prime} \rightarrow \mathrm{g} \mathbb{S} \mathbb{S}^{\prime}\right) \wedge\left(\mathrm{g}_{t}^{\prime}\left(\operatorname{Next}_{\mathrm{c}}(\mathbb{S})\right) \mathbb{S}^{\prime} \rightarrow \mathrm{g}_{t} \mathbb{S} \mathbb{S}^{\prime}\right)
\end{array} \\
& \vdash\left\{\left\langle\llbracket\left(\mathrm{p}, \mathrm{g}, \mathrm{g}_{t}, \mathrm{~g}_{r}\right)_{i} \rrbracket\right\rangle_{\Psi_{\mathrm{L}}}\right\} \mathrm{c} ; \mathbb{I}
\end{aligned}
$$

In SCAP-CR, we need to enforce the invariant on two wellformed control stacks, as we did in SCAP. The interpretation function for the specification $\left(\mathrm{p}, \mathrm{g}, \mathrm{g}_{t}, \mathrm{~g}_{r}\right)_{i}$ is defined in Figure 35. The predicate WFCR ensures that:

- there is a well formed control stack for the current coroutine;

- at the switch point, \$ry contains a valid code pointer;

- the precondition of the $\$ r y$ to which we are switching is satisfied at the switch point, i.e., $\mathrm{p}^{\prime} \mathbb{S}^{\prime}$

- the code to which we are switching will satisfy the coroutine $(1-i)$ 's global guarantee, i.e., $\mathrm{g}_{t}^{\prime} \Rightarrow \mathbb{G}_{1-i}$; and

- at the switch point, there is a well-formed control stack in the coroutine $(1-i)$. 


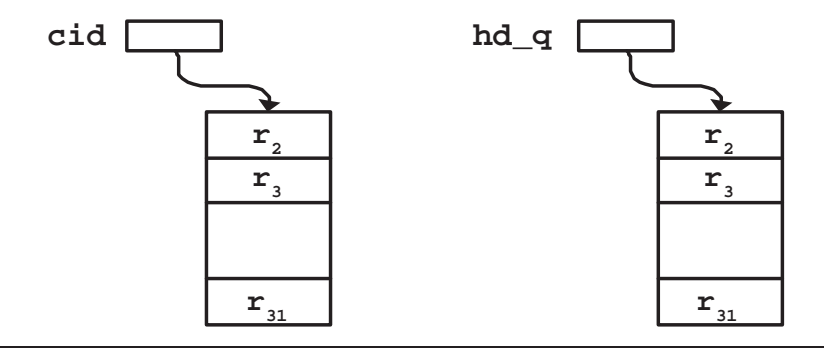

Figure 36. Organization of TCB

$$
\begin{aligned}
& \text { (Assertion) p } \in \text { State } \rightarrow \text { Prop } \\
& \text { (Guarantee) } \mathrm{g} \in \text { State } \rightarrow \text { State } \rightarrow \text { Prop } \\
& \text { (Assumption) } \mathbb{A} \in \text { RegFile } \rightarrow \text { Heap } \rightarrow \text { Heap } \rightarrow \text { Prop } \\
& (\text { Th-Guarant. }) \breve{\mathrm{g}}, \mathbb{G} \in \text { RegFile } \rightarrow \text { Heap } \rightarrow \text { Heap } \rightarrow \text { Prop } \\
& \text { (CdSpec) } \quad \theta \quad::=(\mathrm{p}, \mathrm{g}) \mid(\mathrm{p}, \breve{\mathrm{g}}, \mathbb{A}, \mathbb{G})
\end{aligned}
$$

Figure 37. Code Specifications in Foundational CCAP

The definition of the well-formed control stack is similar to the definition of WFST in SCAP, except we also need to ensure that the caller's behavior from the return point to the next switch point actually satisfies the callee's expected behavior, i.e., $\mathrm{g}_{t}^{\prime} \Rightarrow \mathrm{g}_{r}$.

As usual, inference rules in SCAP-CR are provable as CAP0 lemmas based on this interpretation function.

\section{Foundational CCAP}

In the implementation of thread libraries, the routine for thread context-switching does not follow the regular calling convention: it fetches the return code pointer from the target thread's stack and returns to the target thread instead of the calling thread. It was believed [41] that support of general first-class code pointers are required to reason about the context-switch routine.

In previous work $[41,13]$, we applied the rely-guarantee method in to support thread-modular verification of concurrent assembly code. There threads yield by executing a "yield" pseudo instruction instead of making a function call to a certified implementation of a "yield" function, which involves certifying the context-switching code.

In this section, we show how to use SCAP-like rules to certify a simple implementation of "yield", which can be linked in CAP0 with certified user-level CCAP [41] thread code. To simplify the presentation, we work on the two-thread version of CCAP. This work allows us to generate foundational PCC for real concurrent machine code (instead of code for the abstract CCAP machine). It also illustrate how different reasoning methodologies can be interfaced in CAP0.

Figure 36 shows the organization of thread control blocks (TCBs) in memory. Each TCB contains the saved register file (except $r_{0}$ and $r_{1}$ ) of the thread. The global variable cid contains the pointer that points to the current thread's TCB, while hd_q contains the pointer that points to the thread queue. Since there are always two threads in the system, only one thread is in the thread queue.

Code specifications are defined in Figure 37. We use two kinds of specifications: SCAP specification $(p, g)$ for the yield function, and CCAP specification $(p, \breve{g}, \mathbb{A}, \mathbb{G})$ for user thread code. Here $\mathrm{p}$ and $\mathrm{g}$ has the same meaning with those in SCAP. The local guarantee $\breve{g}$ is used in CCAP to support non-preemptive thread model. Similar to $g_{t}$ in CAP-CR, $\breve{g}$ describes the behavior of code segments from the current instruction to the next "yield" point. The assumption $\mathbb{A}$ and global guarantee $\mathbb{G}$ describe the state transition

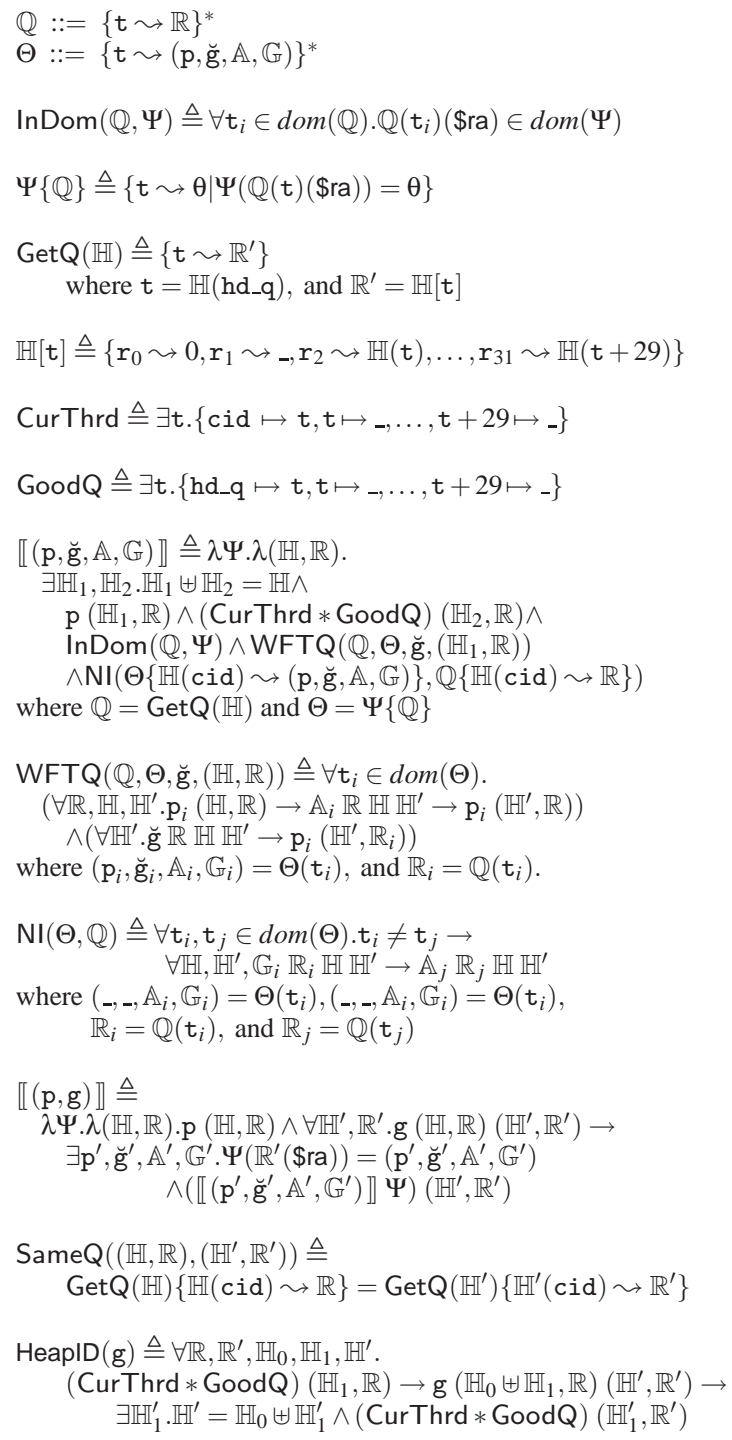

Figure 38. Definition of Macros for CCAP

between two "yield" points. For each thread, if state transitions made by the environment satisfy the assumption $\mathbb{A}$, the thread's transitions will meet its guarantee $\mathbb{G}$ to the environment. The $\mathbb{G}$ here has the same meaning with the one in CAP-CR. However, since we save the register file during thread context-switch, register files are thread-private data. Therefore $\breve{g}, \mathbb{A}$ and $\mathbb{G}$ only specify the relation between a pair of shared data heaps. They are also parameterized with the current register file. To distinguish them from the guarantees used in SCAPand CAP-CR, we call $\breve{g}, \mathbb{A}$ and $\mathbb{G}$ in FCCAP "Thread Guarantee".

Figure 38 shows the predicates and macros used to define interpretations of code specifications. $\mathbb{Q}$ is the abstract thread queue, which maps thread id to its register file saved in its TCB. We use the pointer to the TCB as the thread id. $\Theta$ maps each thread to its specification. The predicate $\operatorname{InDom}$ requires the return code pointer saved in each TCB be a valid code pointer. $\Psi\{\mathbb{Q}\}$ extracts the specification for the return code pointer of each thread in $\mathbb{Q}$ from $\Psi$.

GetQ $(\mathbb{H})$ extracts TCBs in the thread queue pointed to by hd_q and returns the abstract queue $\mathbb{Q}$. The predicate CurThrd says that cid points to the TCB of the current executing thread, while 
GoodQ requires hd_q point to a list of TCBs. In our case there is only one TCB in the list.

The interpretation for $(p, \breve{g}, \mathbb{A}, \mathbb{G})$ requires the data heap can be split into two disjoint parts: $\mathbb{H}_{1}$ and $\mathbb{H}_{2}$. $\mathbb{H}_{1}$ is the user thread heap and satisfies $\mathrm{p}$ which has no knowledge of $\mathbb{H}_{2}$, while $\mathbb{H}_{2}$ contains TCBs for the current thread and threads in the queue. The separating conjunction $P * Q$ is borrowed from separation logic, which says $P$ and $Q$ hold for disjoint portions of the data heap. The interpretation function also encodes the invariant enforced in original CCAP:

- the return code pointer for each thread in the queue is valid;

- when the current thread yields, threads in the queue can take control from their return code pointers (defined in WFTQ); and

- threads do not interfere with each other (defined in NI).

Interested readers can refer to CCAP [41] for more details.

The interpretation for $(\mathrm{p}, \mathrm{g})$ is straightforward. It simply requires that, when the function returns, there be a valid return code pointer in \$ra pointing to the user thread's code, and that the interpretation of its specification hold.

In our system, the yield instruction in original CCAP is replaced with a function call to the yield function. The YIELD rule combines the original YIELD rule in CCAP and the CALL rule in SCAP, and bridges the system call from CCAP code to SCAP code.

$$
\begin{aligned}
& \text { yield, } \mathrm{f}_{\text {ret }} \in \operatorname{dom}(\Psi) \\
& \left(\mathrm{p}_{y}, \mathrm{~g}_{y}\right)=\Psi(\text { yield }) \quad(\mathrm{p}, \mathbb{G}, \mathbb{A}, \mathbb{G})=\Psi\left(\mathrm{f}_{\text {ret }}\right) \\
& \forall \mathbb{R}, \mathbb{H}, \mathbb{H}^{\prime}, \mathrm{p}(\mathbb{H}, \mathbb{R}) \rightarrow \mathbb{A} \mathbb{R} \mathbb{H}_{\mathbb{H}} \mathbb{H}^{\prime} \rightarrow \mathrm{p}\left(\mathbb{H}^{\prime}, \mathbb{R}\right) \\
& \forall \mathbb{R}, \mathbb{H} . \mathrm{p}(\mathbb{H}, \mathbb{R}) \rightarrow \breve{\mathrm{g}} \mathbb{R} \mathbb{H} \mathbb{H} \\
& \forall \mathbb{S}, \mathbb{S}^{\prime} . \mathrm{g}_{y} \mathbb{S} \mathbb{S}^{\prime} \rightarrow \operatorname{SameQ}\left(\mathbb{S}, \mathbb{S}^{\prime}\right) \quad \text { HeaplD }\left(\mathrm{g}_{y}\right) \\
& \forall \mathbb{S} \text {. }(\mathrm{p} * \operatorname{CurThrd} * \operatorname{GoodQ}) \mathbb{S} \rightarrow \mathrm{p}_{y} \mathbb{S} \\
& \vdash\left\{\langle\llbracket(\mathrm{p}, \breve{\mathrm{g}}, \mathbb{A}, \mathbb{G}) \rrbracket\rangle_{\Psi}\right\} \text { jal yield, } \mathrm{f}_{\text {ret }}
\end{aligned}
$$

The first two lines show specifications for the function yield and the return code pointer. The premise in line 3 says the current thread can take control after any state transitions satisfying $\mathbb{A}$. Note that the register file is not changed during the state transition. Line 4 says the current thread has finished the state transition which satisfies its guarantee $\breve{g}$. These two premises are adapted from the original YIELD rule in CCAP. The last three premises are similar to those in the CALL rule of SCAP. The first premise in line 5 requires that the yield function save the current thread's register file and restore the register file for the thread which is scheduled to run. The predicate HeapID $\left(g_{y}\right)$ requires the yield function not touch the user thread heap. Both SameQ $\left(\mathbb{S}, \mathbb{S}^{\prime}\right)$ and HeapID $\left(\mathrm{g}_{y}\right)$ are defined in Figure 38. The last premise says the precondition of the yield function holds on the conjunction of the user thread heap and TCBs for threads.

The Y-RET rule is used for yield to return to the user-level thread code. It is almost the same with the RET rule in SCAP.

$$
\frac{\forall \mathbb{S} . p \mathbb{S} \rightarrow \mathrm{g} \mathbb{S} \mathbb{S}}{\vdash\left\{\langle\llbracket(\mathrm{p}, \mathrm{g}) \rrbracket\rangle_{\Psi}\right\} \mathrm{jr} \$ \mathrm{ra}}
$$

In addition to the YIELD and Y-RET rules, for each instruction we have two rules: the original SCAP rule and the original CCAP rule. Depending on the place an instruction is used, the corresponding rule is used to verify it.

In Figure 39 we show the TM code for yield, which essentially implements the context switch routine. The specification is given on the top. Readers can check that it satisfies the premises of the YIELD rule.

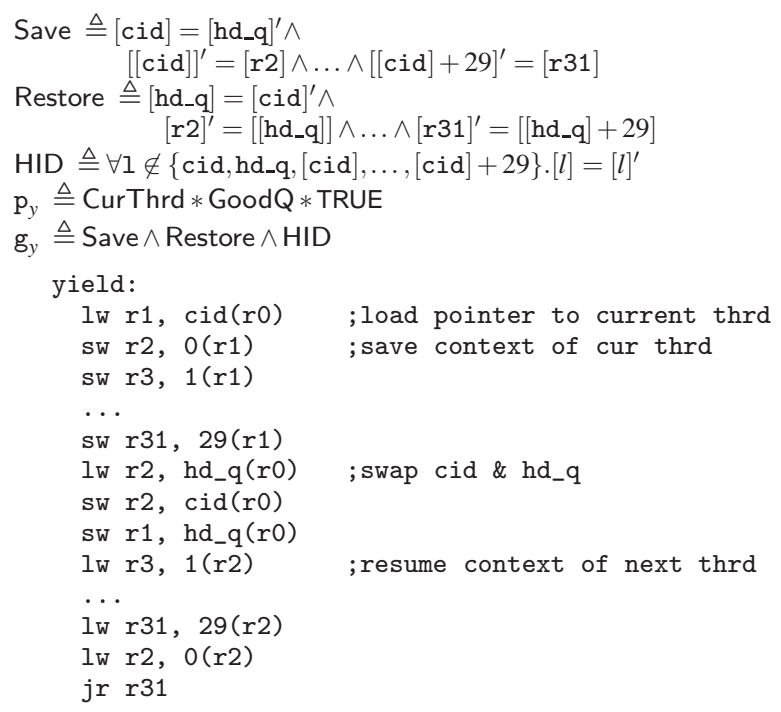

Figure 39. Thread Library Code - Yield

\section{Implementation}

We use the Coq proof assistant [35] and the underlying higher-order predicate logic for fully mechanized verification of assembly code. The syntax of the TM is encoded in Coq using inductive definitions. Operational semantics of TM and the inference rules of CAPO are defined as inductive relations. The soundness of the CAP0 rules is formalized and proved in Coq.

Instead of defining the syntax and semantics of the assertion language (which is known as the deep embedding approach), we use $\mathrm{CiC}$, the underlying higher-order logic in Coq, as our assertion language. This shallow embedding approach greatly reduces the work load of formulating our logic systems.

Our implementation includes around 370 lines of Coq encoding of TM and its operational semantics, 200 lines encoding of CAP0 rules, and 700 lines of Coq tactics for the soundness proof. We also encoded in Coq the definition of SCAP inference rules and their proofs as CAP0 lemmas, which consists of around 900 lines of Coq inductive definitions and tactics. We have written more than 10 thousand lines of Coq tactics to certify practical programs, including the malloc/free library which was first certified in the original CAP [40]. According to our experience, human smartness is required to come up with proper program specifications, the difficulty depending on the property one is interested in and the subtlety of algorithms. Given proper specifications, proof construction of assembly code is mostly routine work. Some premises of SCAP rules can be automatically derived after defining lemmas for common instructions. For generality, we intentionally avoid specifying the layout of the physical stack and calling convention in SCAP. The low abstraction level causes lengthy (but still straightforward) proof for instructions involving memory operations. The burden of the programmer can be reduced if we define higher-level lemmas for specific stack organization. We leave this as the future work.

\section{More Related Work and Conclusion}

Reasoning about Stacks and Exceptions. Continuing over the related work discussed in Section 2.1, STAL [23] and its variations [11, 37] support static type-checking of function call/return and stack unwinding, but they all treat return code pointers as firstclass code pointers and stacks as "closures". Introducing a "ret" instruction [11] does not change this fact because there the typing rule for "ret" requires a valid code pointer on the top of the stack, which is very different from our SCAP RET rule. Impredica- 
tive polymorphism has to be used in these systems to abstract over unused portions of the stack (as a closure), even though only return addresses are stored on the stack. Using compound stacks, STAL can type-check exceptions, but this approach is rather limited. If multiple exception handlers defined at different depths of the stack are passed to the callee, the callee has to specify their order on the stack, which breaks modularity. This problem may be overcome by using intersection types [11], though it has never been shown. Moreover, there is no known work certifying setjmp/longjmp and weak continuations using these systems.

Also, unlike STAL, SCAP does not require any built-in stack structure in the target machine (TM), so it does not need two sets of instructions for heap and stack operations. As shown in Figure 13, SCAP can easily support general data pointers into the stack or heap, which are not supported in STAL. In addition, SCAP does not enforce any specific stack layout, therefore it can be used to support sequential stacks, linked stacks, and heap-allocated activation records.

Concurrently with our work, Benton [5] proposed a typed program logic for a stack-based abstract machine. His instruction sequence specification is similar to the $\mathrm{g}$ in SCAP. Typing rules in his system also look similar to SCAP rules. However, to protect return code pointers, Benton uses a higher-level abstract machine with separate data stack and control stack; the latter cannot be touched by regular instructions except call and ret. Benton also uses a pair of pre- and postcondition as the specification which requires complex formalization of auxiliary variables.

At higher-level, Berdine et al. [6] showed that function call and return, exceptions, goto statements and coroutines follow a discipline of linearly used continuations. The idea is formalized by typing continuation transformers as linear functions, but no verification logic was proposed for reasoning about programs. Following the producer/consumer model (in Figure 2), our reasoning has a flavor of linearity, but it is not clear how our work and linear continuation-passing relate to each other.

Walker et al. [1, 17] proposed logical approaches for stack typing. They used CPS to reason about function calls. Their work focused on memory management and alias reasoning, while in SCAP we left the stack layout unspecified. Although the higher-order predicate logic is general enough to specify memory properties, substructural logic provides much convenience for memory specification. Applying their work to provide lemmas for different stack layouts and calling conventions will be our future work.

Reasoning about First-Class Code Pointers. Ni and Shao [29] introduce a special syntax $\operatorname{cptr}(\mathrm{f}, \mathrm{a})$ in their assertion language to certify first-class code pointers. To support first-class code pointers in SCAP, we can extend it in a similar way by using $\operatorname{cptr}(\mathrm{f},(\mathrm{p}, \mathrm{g}))$, which means $f$ is a function pointer with the specification $(p, g)$. However, as we mentioned before, return code pointers and exception handlers have subtly different invariants from general firstclass code pointers. So even with the support of first-class code pointers, it is still desirable to not treat regular stack-based control abstractions as general code pointers. Embedding SCAP and its extensions into the CAP0 framework allows interoperability between SCAP and other systems. We can reason about function call/return, exception handling, and coroutine as before and then use $\operatorname{cptr}(\mathrm{f},(\mathrm{p}, \mathrm{g}))$ to reason about unavoidable first-class code pointers. Another interesting observation is that some seemingly first-class code pointers, such as threads' return code pointers stored in the thread queue, can actually be reasoned using SCAPbased systems. We need more experience to fully explore the applicability and the limitations of SCAP.

State Relations as Program Specifications. SCAP is not the first to use relations between two states as program specifications. The rely-guarantee method [18], TLA [21], and VDM [19] all use state relations to specify programs. However, the guarantee $g$ used in SCAP is different from those used in previous systems. Generalizing the idea of local guarantee [41], SCAP uses g to describe the obligation that the current function must fulfill before it can return, raise an exception, or switch to other coroutines and threads. Notice that at the beginning of a function, our $g$ matches precisely the VDM postcondition, but intermediate g's used in our SCAP-SEQ rule differ from the intermediate postconditions used in the sequential decomposition rule in VDM: the second state specified in our g's always refers to the (same) state at the exit point. We use these intermediate g's to bridge the gap between the entry and exit points of functions - this is hard to achieve using VDM's post conditions.

Yu's pioneer work [42] on machine code verification can also support stack-based procedure call and return. His correctness theorem for each subroutine resembles our guarantee $\mathrm{g}$, but it requires auxiliary logical predicates counting the number of instructions executed between different program points. It is unclear whether their method can be extended to handle complex stack-based controls as discussed in our current paper.

Conclusion. We have proposed a new methodology for modular verification of assembly code with all kinds of stack-based control abstractions, including function call/return, tail call, weak continuation, setjmp/longjmp, stack cutting, stack unwinding, multireturn function call, coroutines, and thread context switch. For each control abstraction, we have formalized its invariants and showed how to certify its implementation. All reasoning systems are proposed as instances of the generic CAP0 framework, which allows programs certified in different PCC systems to be linked together. Our system is fully mechanized [36]: we give the complete soundness proof and a full verification of several examples in the Coq proof assistant [35].

\section{References}

[1] A. Ahmed and D. Walker. The logical approach to stack typing. In Proc. of the 2003 ACM SIGPLAN Int'l workshop on Types in Lang. Design and Impl., pages 74-85. ACM Press, 2003.

[2] A. W. Appel. Compiling with Continuations. Cambridge University Press, New York, 1992.

[3] A. W. Appel. Foundational proof-carrying code. In Symp. on Logic in Comp. Sci. (LICS'01), pages 247-258. IEEE Comp. Soc., June 2001.

[4] K. R. Apt. Ten years of Hoare's logic: A survey - part I. ACM Trans. on Programming Languages and Systems, 3(4):431-483, 1981.

[5] N. Benton. A typed, compositional logic for a stack-based abstract machine. In Proc. Third Asian Symp. on Prog. Lang. and Sys., LNCS 3780, pages 364-380. Springer-Verlag, November 2005.

[6] J. Berdine, P. O'hearn, U. Reddy, and H. Thielecke. Linear continuation-passing. Higher Order Symbol. Comput., 15(2-3):181208, 2002.

[7] D. Chase. Implementation of exception handling, Part I. The Journal of C Language Translation, 5(4):229-240, June 1994.

[8] W. D. Clinger. Proper tail recursion and space efficiency. In Proc. 1997 ACM Conf. on Prog. Lang. Design and Impl., pages 174-185, New York, 1997. ACM Press.

[9] C. Colby, P. Lee, G. Necula, F. Blau, M. Plesko, and K. Cline. A certifying compiler for Java. In Proc. 2000 ACM Conf. on Prog. Lang. Design and Impl., pages 95-107, New York, 2000. ACM Press.

[10] M. E. Conway. Design of a separable transition-diagram compiler. Communications of the ACM, 6(7):396-408, July 1963.

[11] K. Crary. Toward a foundational typed assembly language. Technical Report CMU-CS-02-196, Carnegie Mellon University, School of Computer Science, Dec. 2002. 
[12] S. J. Drew, J. Gough, and J. Ledermann. Implementing zero overhead exception handling. Technical Report 95-12, Faculty of Information Technology, Queensland U. of Technology, Brisbane, Australia, 1995.

[13] X. Feng and Z. Shao. Modular verification of concurrent assembly code with dynamic thread creation and termination. In Proc. 2005 ACM SIGPLAN Int'l Conf. on Functional Prog., pages 254-267. ACM Press, September 2005.

[14] N. A. Hamid, Z. Shao, V. Trifonov, S. Monnier, and Z. Ni. A syntactic approach to foundational proof-carrying code. In Proc. Seventeenth Annual IEEE Symposium on Logic In Computer Science (LICS'02), pages 89-100. IEEE Computer Society, July 2002.

[15] D. R. Hanson. C Interface \& Implementations. Add. Wesley, 1997.

[16] C. A. R. Hoare. An axiomatic basis for computer programming. Communications of the ACM, 12(10):576-580, Oct. 1969.

[17] L. Jia, F. Spalding, D. Walker, and N. Glew. Certifying compilation for a language with stack allocation. In Proc. 20th IEEE Symposium on Logic in Computer Science, pages 407-416, June 2005.

[18] C. B. Jones. Tentative steps toward a development method for interfering programs. ACM Trans. on Programming Languages and Systems, 5(4):596-619, 1983.

[19] C. B. Jones. Systematic software development using VDM. Prentice Hall International (UK) Ltd., 1986.

[20] B. W. Kernighan and D. M. Ritchie. The C Programming Language (Second Edition). Prentice Hall, 1988.

[21] L. Lamport. The temporal logic of actions. ACM Trans. on Programming Languages and Systems, 16(3):872-923, May 1994.

[22] T. Lindholm and F. Yellin. The Java Virtual Machine Specification (Second Edition). Addison-Wesley, 1999.

[23] G. Morrisett, K. Crary, N. Glew, and D. Walker. Stack-based typed assembly language. In Proc. 1998 Int'l Workshop on Types in Compilation: LNCS Vol 1473, pages 28-52. Springer-Verlag, 1998.

[24] G. Morrisett, D. Walker, K. Crary, and N. Glew. From System F to typed assembly language. In Proc. 25th ACM Symp. on Principles of Prog. Lang., pages 85-97. ACM Press, Jan. 1998.

[25] D. A. Naumann. Predicate transformer semantics of a higher-order imperative language with record subtyping. Science of Computer Programming, 41(1):1-51, 2001.

[26] G. Necula. Compiling with Proofs. PhD thesis, School of Computer Science, Carnegie Mellon Univ., Sept. 1998.

[27] G. Necula and P. Lee. The design and implementation of a certifying compiler. In Proc. 1998 ACM Conf. on Prog. Lang. Design and Impl., pages 333-344, New York, 1998.

[28] Z. Ni and Z. Shao. A translation from typed assembly languages to certified assembly programming. Technical report, Dept. of Computer Science, Yale Univ., New Haven, CT, Nov. 2005. http: //flint.cs.yale.edu/flint/publications/talcap.html.

[29] Z. Ni and Z. Shao. Certified assembly programming with embedded code pointers. In Proc. 33rd ACM Symp. on Principles of Prog. Lang., pages 320-333, Jan. 2006.

[30] N. Ramsey and S. P. Jones. A single intermediate language that supports multiple implementations of exceptions. In Proc. 2000 ACM Conf. on Prog. Lang. Design and Impl., pages 285-298, 2000.

[31] J. C. Reynolds. Separation logic: A logic for shared mutable data structures. In Proc. 17th Annual IEEE Symposium on Logic in Computer Science, pages 55-74. IEEE Computer Society, 2002.

[32] O. Shivers and D. Fisher. Multi-return function call. In Proc. 2004 ACM SIGPLAN Int'l Conf. on Functional Prog., pages 79-89. ACM Press, Sept. 2004.

[33] R. Stata and M. Abadi. A type system for java bytecode subroutines. In Proc. 25th ACM Symp. on Principles of Prog. Lang., pages 149160. ACM Press, 1998.

[34] G. L. Steele. Rabbit: a compiler for Scheme. Technical Report AI-TR-474, MIT, Cambridge, MA, 1978.
[35] The Coq Development Team. The Coq proof assistant reference manual. The Coq release v8.0, Oct. 2004.

[36] The Flint Group. Coq (v8.0) implementation for the CAP framework and SCAP. http://flint.cs.yale.edu/publications/sbca. html, July 2005.

[37] J. C. Vanderwaart and K. Crary. A typed interface for garbage collection. In Proc. 2003 ACM SIGPLAN International Workshop on Types in Language Design and Implementation, pages 109-122, 2003.

[38] D. von Oheimb. Hoare logic for Java in Isabelle/HOL. Concurrency and Computation: Practice and Experience, 13(13):1173-1214, 2001.

[39] A. K. Wright and M. Felleisen. A syntactic approach to type soundness. Information and Computation, 115(1):38-94, 1994.

[40] D. Yu, N. A. Hamid, and Z. Shao. Building certified libraries for PCC: Dynamic storage allocation. In Proc. 2003 European Symposium on Programming, LNCS Vol. 2618, pages 363-379, 2003.

[41] D. Yu and Z. Shao. Verification of safety properties for concurrent assembly code. In Proc. 2004 ACM SIGPLAN Int'l Conf. on Functional Prog., pages 175-188, September 2004.

[42] Y. Yu. Automated Proofs of Object Code For A Widely Used Microprocessor. PhD thesis, University of Texas at Austin, 1992.

\section{A. Soundness of SCAP, SCAP-I and SCAP-II}

In this section, we prove the soundness of inference rules in SCAP, SCAP-I and SCAP-II by showing that they can be derived from CAP0 rules. We only show the derivation of the most important rules, i.e., the CALL and RET rules in SCAP, the CALL-I rule in SCAP-I and CALL-II rule in SCAP-II. Derivations for other inference rules in each system are similar and omitted here. We also encoded the derivation of the complete set of SCAP rules in the Coq proof assistant, which is available at [36].

Lemma A.1 (Stack Strengthen) For all $n, \mathrm{~g}, \mathrm{~g}^{\prime}, \mathbb{S}, \mathbb{S}^{\prime}$ and $\Psi$, if

$$
\operatorname{WFST}(n, \mathrm{~g}, \mathbb{S}, \Psi) \text { and } \forall \mathbb{S}^{\prime \prime} \cdot \mathrm{g}^{\prime} \mathbb{S}^{\prime} \mathbb{S}^{\prime \prime} \rightarrow \mathrm{g} \mathbb{S} \mathbb{S}^{\prime \prime},
$$

we have $\operatorname{WFST}\left(n, \mathrm{~g}^{\prime}, \mathbb{S}^{\prime}, \Psi\right)$.

Proof. This trivially follows the definition of WFST.

Lemma A.2 (Call) Suppose $\mathrm{f}, \mathrm{f}_{\text {ret }} \in \operatorname{dom}\left(\Psi_{\mathrm{L}}\right),\left(\mathrm{p}^{\prime}, \mathrm{g}^{\prime}\right)=\Psi_{\mathrm{L}}(\mathrm{f})$ and $\left(\mathrm{p}^{\prime \prime}, \mathrm{g}^{\prime \prime}\right)=\Psi_{\mathrm{L}}\left(\mathrm{f}_{\text {ret }}\right)$. If

1. $\forall \mathbb{H}, \mathbb{R} \cdot \mathrm{p}(\mathbb{H}, \mathbb{R}) \rightarrow \mathrm{p}^{\prime}\left(\mathbb{H}, \mathbb{R}\left\{\$ \mathrm{ra} \leadsto \mathrm{f}_{\text {ret }}\right\}\right) ;$

2. $\forall \mathbb{H}, \mathbb{R}, \mathbb{S}^{\prime} \cdot \mathrm{p}(\mathbb{H}, \mathbb{R}) \rightarrow \mathrm{g}^{\prime}\left(\mathbb{H}, \mathbb{R}\left\{\right.\right.$ \$ra $\left.\left.\sim \mathrm{f}_{\text {ret }}\right\}\right) \mathbb{S}^{\prime} \rightarrow$

$\left(\mathrm{p}^{\prime \prime} \mathbb{S}^{\prime} \wedge\left(\forall \mathbb{S}^{\prime \prime} \cdot \mathrm{g}^{\prime \prime} \mathbb{S}^{\prime} \mathbb{S}^{\prime \prime} \rightarrow \mathrm{g}(\mathbb{H}, \mathbb{R}) \mathbb{S}^{\prime \prime}\right)\right)$

3. $\forall \mathbb{S}, \mathbb{S}^{\prime} \cdot \mathrm{g}^{\prime} \mathbb{S} \mathbb{S}^{\prime} \rightarrow \mathbb{S} \cdot \mathbb{R}(\$ r a)=\mathbb{S}^{\prime} \cdot \mathbb{R}(\$ r a)$;

we have

$$
\begin{aligned}
\forall \Psi, \mathbb{H}, \mathbb{R} .\langle[[(\mathrm{p}, \mathrm{g})]]\rangle \Psi_{\mathrm{L}} \Psi(\mathbb{H}, \mathbb{R}) \rightarrow \\
{[[\Psi(\mathrm{f})]] \Psi\left(\mathbb{H}, \mathbb{R}\left\{\$ \mathrm{ra} \leadsto \mathrm{f}_{\text {ret }}\right\}\right) . }
\end{aligned}
$$

(In short, the CALL rule can be derived from the JAL rule).

Proof. Unfolding the definition of the interpretation function, we know that, given

4. $\Psi_{\mathrm{L}} \subseteq \Psi$;

5. $\mathrm{p}(\mathbb{H}, \mathbb{R})$;

6. $\operatorname{WFST}(n, \mathrm{~g},(\mathbb{H}, \mathbb{R}), \Psi)$;

we need to prove

a. $\mathrm{p}^{\prime}\left(\mathbb{H}, \mathbb{R}\left\{\right.\right.$ \$ra $\left.\left.\leadsto \mathbf{f}_{\text {ret }}\right\}\right)$; and

b. $\operatorname{WFST}\left(n+1, \mathrm{~g}^{\prime},\left(\mathbb{H}, \mathbb{R}\left\{\$\right.\right.\right.$ ra $\left.\left.\left.\sim \mathrm{f}_{\text {ret }}\right\}\right), \Psi\right)$;

The proof of a is trivial (by 1 and 5). We focus on the proof of $b$.

By 4 and the assumption, we know that $\mathrm{f}, \mathrm{f}_{\text {ret }} \in \operatorname{dom}(\Psi), \Psi(\mathrm{f})=$ $\left(\mathrm{p}^{\prime}, \mathrm{g}^{\prime}\right)$ and $\Psi\left(\mathrm{f}_{\text {ret }}\right)=\left(\mathrm{p}^{\prime \prime}, \mathrm{g}^{\prime \prime}\right)$. For all $\mathbb{S}$, if $\mathrm{g}^{\prime}\left(\mathbb{H}, \mathbb{R}\left\{\$\right.\right.$ ra $\left.\left.\sim \mathrm{f}_{\text {ret }}\right\}\right) \mathbb{S}$, 
- by 3 we know $\mathbb{S} . \mathbb{R}(\$ r a)=\mathrm{f}_{\text {ret }}$, therefore $\mathbb{S} . \mathbb{R}(\$ r a) \in \operatorname{dom}(\Psi)$;

- by 5 and 2 we know $\mathrm{p}^{\prime \prime} \mathbb{S}$;

- by $5,2,6$, and Lemma A.1 we know $\operatorname{WFST}\left(n, \mathrm{~g}^{\prime \prime}, \mathbb{S}, \Psi\right)$.

Then, by the definition of WFST we get

$$
\operatorname{WFST}\left(n+1, \mathrm{~g}^{\prime},\left(\mathbb{H}, \mathbb{R}\left\{\text { \$ra } \sim \mathrm{f}_{\text {ret }}\right\}\right), \Psi\right) .
$$

Lemma A.3 (Return) If $\forall \mathbb{S} . p \mathbb{S} \rightarrow \mathrm{g} \mathbb{S} \mathbb{S}$, then for all $\Psi, \mathbb{H}$ and $\mathbb{R}$, we have

$$
[[(\mathrm{p}, \mathrm{g})]] \Psi(\mathbb{H}, \mathbb{R}) \rightarrow[[\Psi(\mathbb{R}(\$ \mathrm{ra}))] \Psi(\mathbb{H}, \mathbb{R}) .
$$

That is, the RET rule can be derived from an instantiation of the JR rule, where $r_{s}$ is instantiated to \$ra.

Proof. Given $[[(\mathrm{p}, \mathrm{g})]] \Psi(\mathbb{H}, \mathbb{R})$ and our assumption, we know that

1. $\mathrm{p}(\mathbb{H}, \mathbb{R})$;

2. $\mathrm{g}(\mathbb{H}, \mathbb{R})(\mathbb{H}, \mathbb{R})$; and

3. $\operatorname{WFST}(n, \mathrm{~g},(\mathbb{H}, \mathbb{R}), \Psi)$ for some $n$.

By 2, 3 and the definition of WFST we know that $n>0$. Therefore, according to the definition of WFST, we can prove

4. $\mathbb{R}(\$ r a) \in \operatorname{dom}(\Psi)$;

5. $\mathrm{p}^{\prime}(\mathbb{H}, \mathbb{R})$;

6. $\operatorname{WFST}\left(n-1, \mathrm{~g}^{\prime},(\mathbb{H}, \mathbb{R}), \Psi\right)$;

where $\left(\mathrm{p}^{\prime}, \mathrm{g}^{\prime}\right)=\Psi(\mathbb{R}(\$ \mathrm{ra}))$. By the definition of the interpretation function, we know $[[\Psi(\mathbb{R}(\$ r a))]] \Psi(\mathbb{H}, \mathbb{R})$.

Lemma A.4 (Call-I) Suppose $f \in \operatorname{dom}\left(\Psi_{\mathrm{L}}\right)$ and $\left(\mathrm{p}^{\prime}, \mathrm{g}^{\prime}\right)=\Psi_{\mathrm{L}}(\mathrm{f})$. If

1. $\forall \mathbb{S} . \mathrm{p} \mathbb{S} \rightarrow \mathrm{p}^{\prime} \mathbb{S}$;

2. $\forall \mathbb{S}, \mathbb{S}^{\prime} \cdot \mathrm{p} \mathbb{S} \rightarrow \mathrm{g}^{\prime} \mathbb{S} \mathbb{S}^{\prime} \rightarrow$

$\mathbb{S}^{\prime} \cdot \mathbb{R}(\$ \mathrm{ra}) \in \operatorname{dom}\left(\Psi_{\mathrm{L}}\right) \wedge \mathrm{p}^{\prime \prime} \mathbb{S}^{\prime} \wedge\left(\forall \mathbb{S}^{\prime \prime} \cdot \mathrm{g}^{\prime \prime} \mathbb{S}^{\prime} \mathbb{S}^{\prime \prime} \rightarrow \mathrm{g} \mathbb{S}^{\prime \prime}\right)$ where $\left(\mathrm{p}^{\prime \prime}, \mathrm{g}^{\prime \prime}\right)=\Psi_{\mathrm{L}}\left(\mathbb{S}^{\prime} \cdot \mathbb{R}(\$ \mathrm{ra})\right)$;

we have

$$
\forall \Psi, \mathbb{S} .\langle[[(\mathrm{p}, \mathrm{g})]]\rangle_{\Psi_{\mathrm{L}}} \Psi \mathbb{S} \rightarrow[[\Psi(\mathrm{f})]] \Psi \mathbb{S} .
$$

(In short, the CALL-I rule is derivable from the $\mathrm{J}$ rule).

Proof. Unfolding the definition of the interpretation function, we know that, given

3. $\Psi_{\mathrm{L}} \subseteq \Psi$;

4. $\mathrm{p} \mathbb{S}$;

5. $\operatorname{WFST}(n, \mathrm{~g}, \mathbb{S}, \Psi)$;

we need prove

a. $\mathrm{p}^{\prime} \mathbb{S}$; and

b. $\operatorname{WFST}\left(n+1, \mathrm{~g}^{\prime}, \mathbb{S}, \Psi\right)$;

The proof of a is trivial (by 1 and 4 ). We focus on the proof of $b$.

For all $\mathbb{S}^{\prime}$, if $\mathrm{g}^{\prime} \mathbb{S} \mathbb{S}^{\prime}$,

- by 4,2 and 3 we know $\mathbb{S}^{\prime} \cdot \mathbb{R}(\$ r a) \in \operatorname{dom}(\Psi)$ and $\left(\mathrm{p}^{\prime \prime}, \mathrm{g}^{\prime \prime}\right)=$ $\Psi\left(\mathbb{S}^{\prime} . \mathbb{R}(\$ r a)\right)$

- by 4 and 2 we know $\mathrm{p}^{\prime \prime} \mathbb{S}^{\prime}$;

- by $4,2,5$, and Lemma A.1 we know $\operatorname{WFST}\left(n, \mathrm{~g}^{\prime \prime}, \mathbb{S}^{\prime}, \Psi\right)$.

Then, by the definition of WFST we get

$$
\operatorname{WFST}\left(n+1, \mathrm{~g}^{\prime}, \mathbb{S}, \Psi\right) \text {. }
$$

Lemma A.5 (Stack Strengthen-II) For all $n, \mathrm{~g}, \mathrm{~g}^{\prime}, \mathbb{S}, \mathbb{S}^{\prime}$ and $\Psi$, if

$$
\operatorname{WFST}^{\prime}(n, \mathrm{~g}, \mathbb{S}, \Psi) \text { and } \forall \mathbb{S}^{\prime \prime} \cdot \mathrm{g}^{\prime} \mathbb{S}^{\prime} \mathbb{S}^{\prime \prime} \rightarrow \mathrm{g} \mathbb{S} \mathbb{S}^{\prime \prime}
$$

we have $\operatorname{WFST}^{\prime}\left(n, \mathrm{~g}^{\prime}, \mathbb{S}^{\prime}, \Psi\right)$.

Proof. This trivially follows the definition of WFST'.

Lemma A.6 (Call-II) Suppose $\mathrm{f} \in \operatorname{dom}\left(\Psi_{\mathrm{L}}\right)$ and $\left(\mathrm{p}^{\prime}, \mathrm{g}^{\prime}\right)=\Psi_{\mathrm{L}}(\mathrm{f})$. If

1. $\forall \mathbb{S} . \mathrm{p} \mathbb{S} \rightarrow \mathrm{p}^{\prime} \mathbb{S}$;

2. $\forall \mathbb{S}, \mathbb{S}^{\prime} \cdot \mathrm{p} \mathbb{S} \rightarrow \mathrm{g}^{\prime} \mathbb{S} \mathbb{S}^{\prime} \rightarrow$ $\left(\mathrm{g} \mathbb{S} \mathbb{S}^{\prime} \vee\right.$

$$
\left.\mathbb{S}^{\prime} \cdot \mathbb{R}(\$ \mathrm{ra}) \in \operatorname{dom}\left(\Psi_{\mathrm{L}}\right) \wedge \mathrm{p}^{\prime \prime} \mathbb{S}^{\prime} \wedge\left(\forall \mathbb{S}^{\prime \prime} \cdot \mathrm{g}^{\prime \prime} \mathbb{S}^{\prime} \mathbb{S}^{\prime \prime} \rightarrow \mathrm{g} \mathbb{S}^{\prime \prime}\right)\right)
$$

where $\left(\mathrm{p}^{\prime \prime}, \mathrm{g}^{\prime \prime}\right)=\Psi_{\mathrm{L}}\left(\mathbb{S}^{\prime} \cdot \mathbb{R}(\$ \mathrm{ra})\right)$;

we have

$$
\forall \Psi, \mathbb{S} .\langle[[(\mathrm{p}, \mathrm{g})]]\rangle_{\Psi_{\mathrm{L}}} \Psi \mathbb{S} \rightarrow[[\Psi(\mathrm{f})]] \Psi \mathbb{S},
$$

where we use the interpretation function defined in Section 5.2, which is different than the one used in Lemma A.4.

Proof. Unfolding the definition of the interpretation function, we know that, given

3. $\Psi_{\mathrm{L}} \subseteq \Psi$;

4. $\mathrm{p} \mathbb{S}$;

5. $\operatorname{WFST}^{\prime}(n, \mathrm{~g}, \mathbb{S}, \Psi)$;

we need prove

a. $\mathrm{p}^{\prime} \mathbb{S}$; and

b. $\exists n^{\prime} . \operatorname{WFST}^{\prime}\left(n^{\prime}, \mathrm{g}^{\prime}, \mathbb{S}, \Psi\right)$;

The proof of a is trivial (by 1 and 4). We focus on the proof of $b$.

For all $\mathbb{S}^{\prime}$, by 4 and 2 we know either

$$
\mathrm{g}^{\prime} \mathbb{S} \mathbb{S}^{\prime} \rightarrow \mathrm{g} \mathbb{S} \mathbb{S}^{\prime}
$$

or

$$
\begin{aligned}
& \mathrm{g}^{\prime} \mathbb{S} \mathbb{S}^{\prime} \rightarrow \\
& \mathbb{S}^{\prime} \cdot \mathbb{R}(\$ \mathrm{ra}) \in \operatorname{dom}\left(\Psi_{\mathrm{L}}\right) \wedge \mathrm{p}^{\prime \prime} \mathbb{S}^{\prime} \wedge\left(\forall \mathbb{S}^{\prime \prime} \cdot \mathrm{g}^{\prime \prime} \mathbb{S}^{\prime} \mathbb{S}^{\prime \prime} \rightarrow \mathrm{g} \mathbb{S}^{\prime \prime}\right) .
\end{aligned}
$$

In the first case, we set $n^{\prime}$ to $n$ and get $\operatorname{WFST}^{\prime}\left(n, \mathrm{~g}^{\prime}, \mathbb{S}, \Psi\right)$ from the Lemma A.5.

In the second case, we set $n^{\prime}$ to $n+1$. The proof of $\mathrm{WFST}^{\prime}(n+$ $\left.1, \mathrm{~g}^{\prime}, \mathbb{S}, \Psi\right)$ is the same with the proof for Lemma A.4.

\section{B. Derivation of EUCAP Inference Rules}

Lemma B.1 (EUCAP Stack Strengthen) For all $n, \mathrm{p}, \mathrm{p}^{\prime}, \mathrm{r}$, and $\Psi$, if $\operatorname{WFSE}(n, \mathrm{p}, r, \Psi)$ and $\forall \mathbb{S} . \mathrm{p}^{\prime} \mathbb{S} \rightarrow \mathrm{p} \mathbb{S}$, we have $\operatorname{WFSE}\left(n, \mathrm{p}^{\prime}, r, \Psi\right)$.

Proof. This trivially follows the definition of WFSE.

Lemma B.2 (EUCAP Stack Strengthen') For all $n, \mathrm{p}, r, r^{\prime}$, and $\Psi$, if $\operatorname{WFSE}(n, \mathrm{p}, r, \Psi)$ and $\forall \mathbb{S} . r^{\prime} \mathbb{S} \rightarrow r \mathbb{S}$, we have $\operatorname{WFSE}\left(n, \mathrm{p}, \mathrm{r}^{\prime}, \Psi\right)$.

Proof. This trivially follows the definition of WFSE.

Lemma B.3 (EUCAP Spec. Strengthen) For all $n, \mathrm{p}, \mathrm{r}, \Psi$ and $\Psi^{\prime}$, if $\operatorname{WFSE}(n, \mathrm{p}, \mathrm{r}, \Psi)$, an $\mathrm{d} \Psi \subseteq \Psi^{\prime}$, we have $\operatorname{WFSE}\left(n, \mathrm{p}, r, \Psi^{\prime}\right)$.

Proof. Induction on $n$.

Lemma B.4 (EU-Call) Suppose $\left.f \in \operatorname{dom}(\Psi),\left(\mathrm{p}^{\prime}, \mathrm{g}^{\prime}, \mathrm{h}^{\prime}\right)=\Psi(\mathrm{f})\right)$. If

1. $\forall \mathbb{H}, \mathbb{R} . \mathrm{p}(\mathbb{H}, \mathbb{R}) \rightarrow \mathrm{p}^{\prime}(\mathbb{H}, \mathbb{R})$ 
2. $\forall \mathbb{H}, \mathbb{R}, \mathbb{S}^{\prime} . \mathrm{p}(\mathbb{H}, \mathbb{R}) \rightarrow \mathrm{g}^{\prime}(\mathbb{H}, \mathbb{R}) \mathbb{S}^{\prime} \rightarrow$

$\mathbb{R}(\$ \mathrm{ra}) \in \operatorname{dom}(\Psi) \wedge \mathrm{p}^{\prime \prime} \mathbb{S}^{\prime} \wedge$

$\left(\forall \mathbb{S}^{\prime \prime} \cdot \mathrm{g}^{\prime \prime} \mathbb{S}^{\prime} \mathbb{S}^{\prime \prime} \rightarrow \mathrm{g}(\mathbb{H}, \mathbb{R}) \mathbb{S}^{\prime \prime}\right) \wedge$

$\left(\forall \mathbb{S}^{\prime \prime} . h^{\prime \prime} \mathbb{S}^{\prime} \mathbb{S}^{\prime \prime} \rightarrow \mathrm{h}(\mathbb{H}, \mathbb{R}) \mathbb{S}^{\prime \prime}\right)$

where $\left(\mathrm{p}^{\prime \prime}, \mathrm{g}^{\prime \prime}, \mathrm{h}^{\prime \prime}\right)=\Psi\left(\mathbb{S}^{\prime} \cdot \mathbb{R}(\right.$ ra $\left.)\right)$

3. $\forall \mathbb{H}, \mathbb{R}, \mathbb{S}^{\prime} . \mathrm{p}(\mathbb{H}, \mathbb{R}) \rightarrow \mathrm{h}^{\prime}(\mathbb{H}, \mathbb{R}) \mathbb{S}^{\prime} \rightarrow$

$\mathbb{R}(\$ r h) \in \operatorname{dom}(\Psi) \wedge \mathrm{p}^{\prime \prime \prime} \mathbb{S}^{\prime} \wedge$

$\left(\forall \mathbb{S}^{\prime \prime} \cdot \mathrm{g}^{\prime \prime \prime} \mathbb{S}^{\prime} \mathbb{S}^{\prime \prime} \rightarrow \mathrm{g}(\mathbb{H}, \mathbb{R}) \mathbb{S}^{\prime \prime}\right) \wedge$

$\left(\forall \mathbb{S}^{\prime \prime} . \mathrm{h}^{\prime \prime \prime} \mathbb{S}^{\prime} \mathbb{S}^{\prime \prime} \rightarrow \mathrm{h}(\mathbb{H}, \mathbb{R}) \mathbb{S}^{\prime \prime}\right)$

where $\left(\mathrm{p}^{\prime \prime \prime}, \mathrm{g}^{\prime \prime \prime}, \mathrm{h}^{\prime \prime \prime}\right)=\Psi\left(\mathbb{S}^{\prime} \cdot \mathbb{R}(\$ \mathrm{rh})\right)$

4. $\forall \mathbb{S}, \mathbb{S}^{\prime} \cdot \mathrm{g}^{\prime} \mathbb{S} \mathbb{S}^{\prime} \rightarrow \mathbb{S} \cdot \mathbb{R}(\$ \mathrm{ra})=\mathbb{S}^{\prime} \cdot \mathbb{R}(\$ \mathrm{ra})$

5. $\forall \mathbb{S}, \mathbb{S}^{\prime} \cdot \mathrm{g}^{\prime} \mathbb{S} \mathbb{S}^{\prime} \rightarrow \mathbb{S} \cdot \mathbb{R}(\$ r h)=\mathbb{S}^{\prime} \cdot \mathbb{R}(\$ r h)$

we have

$\forall \Psi^{\prime}, \mathbb{H}, \mathbb{R} .\langle[[(\mathrm{p}, \mathrm{g}, \mathrm{h})]]\rangle \Psi_{\Psi} \Psi^{\prime}(\mathbb{H}, \mathbb{R}) \rightarrow\left[\left[\Psi^{\prime}(\mathrm{f})\right]\right] \Psi^{\prime}(\mathbb{H}, \mathbb{R})$

(In short, the EU-CALL rule is derivable from the $\mathrm{J}$ rule).

Proof. Unfolding the definition of the interpretation function, we know that, given
6. $\Psi \subseteq \Psi^{\prime}$
7. $\mathrm{p}(\mathbb{H}, \mathbb{R})$
8. $\operatorname{WFSE}\left(n, \mathrm{~g}(\mathbb{H}, \mathbb{R}), \mathrm{h}(\mathbb{H}, \mathbb{R}), \Psi^{\prime}\right)$

we need to prove

a. $\mathrm{p}^{\prime}(\mathbb{H}, \mathbb{R})$

b. $\operatorname{WFSE}\left(n+1, \mathrm{~g}^{\prime}(\mathbb{H}, \mathbb{R}), \mathrm{h}^{\prime}(\mathbb{H}, \mathbb{R}), \Psi^{\prime}\right)$

The proof of a is trivial (by 1 and 7). We focus on the proof of $b$.

For all $\mathbb{S}^{\prime}$, if $\mathrm{g}^{\prime}(\mathbb{H}, \mathbb{R}) \mathbb{S}^{\prime}$,

by 7 and 2 we know:

9. $\mathbb{S}^{\prime} . \mathbb{R}(\$ \mathrm{ra}) \in \operatorname{dom}\left(\Psi^{\prime}\right)$

10. $\mathrm{p}^{\prime \prime} \mathbb{S}^{\prime}$

11. $\forall \mathbb{S}^{\prime \prime} \cdot \mathrm{g}^{\prime \prime} \mathbb{S}^{\prime} \mathbb{S}^{\prime \prime} \rightarrow \mathrm{g}(\mathbb{H}, \mathbb{R}) \mathbb{S}^{\prime \prime}$

12. $\forall \mathbb{S}^{\prime \prime} . \mathrm{h}^{\prime \prime} \mathbb{S}^{\prime} \mathbb{S}^{\prime \prime} \rightarrow \mathrm{h}(\mathbb{H}, \mathbb{R}) \mathbb{S}^{\prime \prime}$

By lemmas B.1 and B.2, from 8, we know

13. $\operatorname{WFSE}\left(n, \mathrm{~g}^{\prime \prime} \mathbb{S}^{\prime}, \mathrm{h}^{\prime \prime} \mathbb{S}^{\prime}, \Psi^{\prime}\right)$

For all $\mathbb{S}^{\prime}$, if $\mathrm{h}^{\prime}(\mathbb{H}, \mathbb{R}) \mathbb{S}^{\prime}$,

by 8 and 3 we know:

14. $\mathbb{S}^{\prime} \cdot \mathbb{R}(\$ r h) \in \operatorname{dom}\left(\Psi^{\prime}\right)$

15. $\mathrm{p}^{\prime \prime \prime} \mathbb{S}^{\prime}$

16. $\forall \mathbb{S}^{\prime \prime} \cdot \mathrm{g}^{\prime \prime \prime} \mathbb{S}^{\prime} \mathbb{S}^{\prime \prime} \rightarrow \mathrm{g}(\mathbb{H}, \mathbb{R}) \mathbb{S}^{\prime \prime}$

17. $\forall \mathbb{S}^{\prime \prime} . \mathrm{h}^{\prime \prime \prime} \mathbb{S}^{\prime} \mathbb{S}^{\prime \prime} \rightarrow \mathrm{h}(\mathbb{H}, \mathbb{R}) \mathbb{S}^{\prime \prime}$

18. $\operatorname{WFSE}\left(n, \mathrm{~g}^{\prime \prime \prime} \mathbb{S}^{\prime}, \mathrm{h}^{\prime \prime \prime} \mathbb{S}^{\prime}, \Psi^{\prime}\right)$ by lemmas B.1 and B.2

By definition of WFSE with $(9,10,13)$ and $(14,15,18)$,

$\operatorname{WFSE}\left(n+1, \mathrm{~g}^{\prime}(\mathbb{H}, \mathbb{R}), \mathrm{h}^{\prime}(\mathbb{H}, \mathbb{R}), \Psi^{\prime}\right)$

Lemma B.5 (EU-Ret) If $\forall \mathbb{S} . p \mathbb{S} \rightarrow \mathrm{g} \mathbb{S} \mathbb{S}$, then for all $\Psi$, $\mathbb{H}$ and $\mathbb{R}$, we have

$$
[[(\mathrm{p}, \mathrm{g}, \mathrm{h})]] \Psi(\mathbb{H}, \mathbb{R}) \rightarrow[[\Psi(\mathbb{R}(\$ \mathrm{ra}))]] \Psi(\mathbb{H}, \mathbb{R}) .
$$

That is, the EU-RET rule is derivable from an instantiation of the JR rule, where $r_{s}$ is instantiated to \$ra.

Proof. Unfolding the definition of the interpretation function, we know that, given

1. $\mathrm{p} \mathbb{S}$

2. $\operatorname{WFSE}(n, \mathrm{~g} \mathbb{S}, \mathrm{h} \mathbb{S}, \Psi)$

we need to prove

a. $\mathrm{p}^{\prime} \mathbb{S}$ b. $\exists m \cdot \operatorname{WFSE}\left(m, \mathrm{~g}^{\prime} \mathbb{S}, \mathrm{h}^{\prime} \mathbb{S}, \Psi\right)$

where $\left(\mathrm{p}^{\prime}, \mathrm{g}^{\prime}, \mathrm{h}^{\prime}\right)=\Psi(\mathbb{R}(\$ \mathrm{ra}))$.

By 1 we know $g \mathbb{S} \mathbb{S}$

Then, either $n=0$ or $n>0$. If $n=0$, then we know $\neg \exists \mathbb{S}^{\prime}$.g $\mathbb{S} \mathbb{S}^{\prime}$.

Therefore, this case is vacuous.

Then $n>0$.

Then by 2 , we know

- $\mathbb{S} . \mathbb{R}(\$ r a) \in \operatorname{dom}(\Psi)$

- $\mathrm{p}^{\prime} \mathbb{S}$

- $\operatorname{WFSE}\left(n-1, \mathrm{~g}^{\prime} \mathbb{S}, \mathrm{h}^{\prime} \mathbb{S}, \Psi\right)$

where $\left(\mathrm{p}^{\prime}, \mathrm{g}^{\prime}, \mathrm{h}^{\prime}\right)=\Psi(\mathbb{R}(\$ \mathrm{ra}))$.

By choosing $m=n-1$, we satisfy both a and $b$.

Lemma B.6 (EU-Raise) If $\forall \mathbb{S} . p \mathbb{S} \rightarrow \mathrm{h} \mathbb{S} \mathbb{S}$, then for all $\Psi, \mathbb{H}$ and $\mathbb{R}$, we have

$$
[[(\mathrm{p}, \mathrm{g}, \mathrm{h})]] \Psi(\mathbb{H}, \mathbb{R}) \rightarrow[[\Psi(\mathbb{R}(\$ \mathrm{rh}))] \Psi(\mathbb{H}, \mathbb{R}) .
$$

That is, the EU-RAISE rule is derivable from an instantiation of the JR rule, where $r_{S}$ is instantiated to \$rh.

Proof. Unfolding the definition of the interpretation function, we know that, given

1. $\mathrm{p} \mathbb{S}$

2. $\operatorname{WFSE}(n, \mathrm{~g} \mathbb{S}, \mathrm{h} \mathbb{S}, \Psi)$

we need to prove

a. $\mathrm{p}^{\prime \prime} \mathbb{S}$

b. $\exists m \cdot \operatorname{WFSE}\left(m, \mathrm{~g}^{\prime \prime} \mathbb{S}, \mathrm{h}^{\prime \prime} \mathbb{S}, \Psi\right)$

where $\left(\mathrm{p}^{\prime \prime}, \mathrm{g}^{\prime \prime}, \mathrm{h}^{\prime \prime}\right)=\Psi(\mathbb{R}(\$ \mathrm{rh}))$.

By 1 we know $\mathrm{h} \mathbb{S} \mathbb{S}$

Then, either $n=0$ or $n>0$. If $n=0$, then we know $\neg \exists \mathbb{S}^{\prime}$.h $\mathbb{S} \mathbb{S}^{\prime}$.

Therefore, this case is vacuous.

Then $n>0$.

Then by 2, we know

- $\mathbb{S} . \mathbb{R}(\$ r h) \in \operatorname{dom}(\Psi)$

- $\mathrm{p}^{\prime} \mathbb{S}$

- $\operatorname{WFSE}\left(n-1, \mathrm{~g}^{\prime} \mathbb{S}, \mathrm{h}^{\prime} \mathbb{S}, \Psi\right)$

where $\left(\mathrm{p}^{\prime}, \mathrm{g}^{\prime}, \mathrm{h}^{\prime}\right)=\Psi(\mathbb{R}(\$ \mathrm{rh}))$.

By choosing $m=n-1$, we satisfy both $a$ and $b$.

\section{Derivation of ECAP Inference Rules}

Lemma C.1 (ECAP Stack Strengthen) For all $n, \mathrm{p}, \mathrm{p}^{\prime}, \mathrm{r}$, and $\Psi$, if $\operatorname{WFSC}(n, \mathrm{p}, r, \Psi)$ and $\forall \mathbb{S} \cdot \mathrm{p}^{\prime} \mathbb{S} \rightarrow \mathrm{p} \mathbb{S}$, we have $\operatorname{WFSC}\left(n, \mathrm{p}^{\prime}, r, \Psi\right)$.

Proof. This trivially follows the definition of WFSC.

Lemma C.2 (ECAP Stack Strengthen') For all $n, \mathrm{p}, \mathrm{r}, \mathrm{r}^{\prime}$, and $\Psi$, if $\operatorname{WFSC}(n, \mathrm{p}, \mathrm{r}, \Psi)$ and $\forall \mathbb{S} . r^{\prime} \mathbb{S} \rightarrow r \mathbb{S}$, we have $\operatorname{WFSC}\left(n, \mathrm{p}, \mathrm{r}^{\prime}, \Psi\right)$.

Proof. This trivially follows the definition of WFSC.

Lemma C.3 (ECAP Spec. Strengthen) For all $n, \mathrm{p}, \mathrm{r}, \Psi$ and $\Psi^{\prime}$, if $\operatorname{WFSC}(n, \mathrm{p}, \mathrm{r}, \Psi)$, an $\mathrm{d} \Psi \subseteq \Psi^{\prime}$, we have $\operatorname{VFSC}\left(n, \mathrm{p}, \mathrm{r}, \Psi^{\prime}\right)$.

Proof. Induction on $n$.

Lemma C.4 (E-Call) Suppose $\left.\mathrm{f} \in \operatorname{dom}(\Psi),\left(\mathrm{p}^{\prime}, \mathrm{g}^{\prime}, \mathrm{h}^{\prime}\right)=\Psi(\mathrm{f})\right)$. If 1. $\forall \mathbb{H}, \mathbb{R} . \mathrm{p}(\mathbb{H}, \mathbb{R}) \rightarrow \mathrm{p}^{\prime}(\mathbb{H}, \mathbb{R})$ 
2. $\forall \mathbb{H}, \mathbb{R}, \mathbb{S}^{\prime} . \mathrm{p}(\mathbb{H}, \mathbb{R}) \rightarrow \mathrm{g}^{\prime}(\mathbb{H}, \mathbb{R}) \mathbb{S}^{\prime} \rightarrow$

$\mathbb{S}^{\prime} \cdot \mathbb{R}(\$ \mathrm{ra}) \in \operatorname{dom}(\Psi) \wedge \mathrm{p}^{\prime \prime} \mathbb{S}^{\prime} \wedge$

$\left(\forall \mathbb{S}^{\prime \prime} \cdot \mathrm{g}^{\prime \prime} \mathbb{S}^{\prime} \mathbb{S}^{\prime \prime} \rightarrow \mathrm{g}(\mathbb{H}, \mathbb{R}) \mathbb{S}^{\prime \prime}\right) \wedge$

$\left(\forall \mathbb{S}^{\prime \prime} . h^{\prime \prime} \mathbb{S}^{\prime} \mathbb{S}^{\prime \prime} \rightarrow \mathrm{h}(\mathbb{H}, \mathbb{R}) \mathbb{S}^{\prime \prime}\right)$

where $\left(\mathrm{p}^{\prime \prime}, \mathrm{g}^{\prime \prime}, \mathrm{h}^{\prime \prime}\right)=\Psi\left(\mathbb{S}^{\prime} \cdot \mathbb{R}(\$ \mathrm{ra})\right)$

3. $\forall \mathbb{S}, \mathbb{S}^{\prime} . \mathrm{p} \mathbb{S} \rightarrow \mathrm{h}^{\prime} \mathbb{S} \mathbb{S}^{\prime} \rightarrow \mathrm{h} \mathbb{S} \mathbb{S}^{\prime}$

4. $\forall \mathbb{S}, \mathbb{S}^{\prime} \cdot \mathrm{g}^{\prime} \mathbb{S} \mathbb{S}^{\prime} \rightarrow \mathbb{S} \cdot \mathbb{R}(\$ \mathrm{ra})=\mathbb{S}^{\prime} \cdot \mathbb{R}(\$ \mathrm{ra})$

we have

$\forall \Psi^{\prime}, \mathbb{H}, \mathbb{R}$

$\langle[[(\mathrm{p}, \mathrm{g}, \mathrm{h})]]\rangle \Psi \Psi^{\prime}(\mathbb{H}, \mathbb{R}) \rightarrow\left[\left[\Psi^{\prime}(\mathrm{f})\right]\right] \Psi^{\prime}(\mathbb{H}, \mathbb{R})$

(In short, the E-CALL rule is derivable from the J rule).

Proof. Unfolding the definition of the interpretation function, we know that, given
5. $\Psi \subseteq \Psi^{\prime}$
6. $\mathrm{p}(\mathbb{H}, \mathbb{R})$
7. $\operatorname{WFSC}\left(n, \mathrm{~g}(\mathbb{H}, \mathbb{R}), \mathrm{h}(\mathbb{H}, \mathbb{R}), \Psi^{\prime}\right)$

we need to prove

a. $\mathrm{p}^{\prime}(\mathbb{H}, \mathbb{R})$

b. $\operatorname{WFSC}\left(n+1, \mathrm{~g}^{\prime}(\mathbb{H}, \mathbb{R}), \mathrm{h}^{\prime}(\mathbb{H}, \mathbb{R}), \Psi^{\prime}\right)$

The proof of a is trivial (by 1). We focus on the proof of $b$. For all $\mathbb{S}^{\prime}$, if $\mathrm{g}^{\prime}(\mathbb{H}, \mathbb{R}) \mathbb{S}^{\prime}$,

by 6 and 2 we know:

8. $\mathbb{S}^{\prime} \cdot \mathbb{R}(\$ r a) \in \operatorname{dom}\left(\Psi^{\prime}\right)$ by 5 and above equality

9. $\mathrm{p}^{\prime \prime} \mathbb{S}^{\prime}$

10. $\forall \mathbb{S}^{\prime \prime} \cdot \mathrm{g}^{\prime \prime} \mathbb{S}^{\prime} \mathbb{S}^{\prime \prime} \rightarrow \mathrm{g}(\mathbb{H}, \mathbb{R}) \mathbb{S}^{\prime \prime}$

11. $\forall \mathbb{S}^{\prime \prime} . \mathrm{h}^{\prime \prime} \mathbb{S}^{\prime} \mathbb{S}^{\prime \prime} \rightarrow \mathrm{h}(\mathbb{H}, \mathbb{R}) \mathbb{S}^{\prime \prime}$

12. $\operatorname{WFSC}\left(n, \mathrm{~g}^{\prime \prime} \mathbb{S}^{\prime}, \mathrm{h}^{\prime \prime} \mathbb{S}^{\prime}, \Psi^{\prime}\right)$ by C.1 and C.2

where $\left(\mathrm{p}^{\prime \prime}, \mathrm{g}^{\prime \prime}, \mathrm{h}^{\prime \prime}\right)=\Psi^{\prime}\left(\mathbb{S}^{\prime} \cdot \mathbb{R}(\right.$ ra $\left.)\right)$

For all $\mathbb{S}^{\prime}$, if $h^{\prime}(\mathbb{H}, \mathbb{R}) \mathbb{S}^{\prime}$,

by $3, \mathrm{~h}(\mathbb{H}, \mathbb{R}) \mathbb{S}^{\prime}$

by 7 we know:

13. $\mathbb{S}^{\prime} \cdot \mathbb{R}(\$ r h) \in \operatorname{dom}\left(\Psi^{\prime}\right)$

14. $\mathrm{p}^{\prime \prime} \mathbb{S}^{\prime}$

15. $\exists m, m<n \wedge \operatorname{WFSC}\left(m, \mathrm{~g}^{\prime \prime} \mathbb{S}^{\prime}, \mathrm{h}^{\prime \prime} \mathbb{S}^{\prime}, \Psi^{\prime}\right)$

16. $\exists m, m<n+1 \wedge \operatorname{WFSC}\left(m, \mathrm{~g}^{\prime \prime} \mathbb{S}^{\prime}, \mathrm{h}^{\prime \prime} \mathbb{S}^{\prime}, \Psi^{\prime}\right)$ by math

where $\left(\mathrm{p}^{\prime \prime}, \mathrm{g}^{\prime \prime}, \mathrm{h}^{\prime \prime}\right)=\Psi^{\prime}\left(\mathbb{S}^{\prime} \cdot \mathbb{R}(\$ \mathrm{rh})\right)$

By definition of WFSC, 12 and 16

$\operatorname{WFSC}\left(n+1, \mathrm{~g}^{\prime}(\mathbb{H}, \mathbb{R}), \mathrm{h}^{\prime}(\mathbb{H}, \mathbb{R}), \Psi^{\prime}\right)$

Lemma C.5 (E-Try) Suppose $f \in \operatorname{dom}(\Psi)$ and $\left.\left(\mathrm{p}^{\prime}, \mathrm{g}^{\prime}, \mathrm{h}^{\prime}\right)=\Psi(\mathrm{f})\right)$. If

1. $\forall \mathbb{H}, \mathbb{R} \cdot \mathrm{p}(\mathbb{H}, \mathbb{R}) \rightarrow \mathrm{p}^{\prime}(\mathbb{H}, \mathbb{R})$

2. $\forall \mathbb{H}, \mathbb{R}, \mathbb{S}^{\prime} \cdot \mathrm{p}(\mathbb{H}, \mathbb{R}) \rightarrow \mathrm{g}^{\prime}(\mathbb{H}, \mathbb{R}) \mathbb{S}^{\prime} \rightarrow$ $\mathbb{S}^{\prime} \cdot \mathbb{R}(\$ \mathrm{ra}) \in \operatorname{dom}(\Psi) \wedge \mathrm{p}^{\prime \prime} \mathbb{S}^{\prime} \wedge$

$\left(\forall \mathbb{S}^{\prime \prime} . \mathrm{g}^{\prime \prime} \mathbb{S}^{\prime} \mathbb{S}^{\prime \prime} \rightarrow \mathrm{g}(\mathbb{H}, \mathbb{R}) \mathbb{S}^{\prime \prime}\right)$

$\left(\forall \mathbb{S}^{\prime \prime} . \mathrm{h}^{\prime \prime} \mathbb{S}^{\prime} \mathbb{S}^{\prime \prime} \rightarrow \mathrm{h}(\mathbb{H}, \mathbb{R}) \mathbb{S}^{\prime \prime}\right)$

where $\left(\mathrm{p}^{\prime \prime}, \mathrm{g}^{\prime \prime}, \mathrm{h}^{\prime \prime}\right)=\Psi\left(\mathbb{S}^{\prime} \cdot \mathbb{R}(\right.$ ra $\left.)\right)$

3. $\forall \mathbb{H}, \mathbb{R}, \mathbb{S}^{\prime}, \mathbb{S}^{\prime \prime} . \mathrm{p}(\mathbb{H}, \mathbb{R}) \rightarrow \mathrm{h}^{\prime}(\mathbb{H}, \mathbb{R}) \mathbb{S}^{\prime} \rightarrow$

$\mathbb{S}^{\prime} \cdot \mathbb{R}(\$ r h) \in \operatorname{dom}(\Psi) \wedge \mathrm{p}^{\prime \prime \prime} \mathbb{S}^{\prime} \wedge$

$\left(\mathrm{g}^{\prime \prime \prime} \mathbb{S}^{\prime} \mathbb{S}^{\prime \prime} \rightarrow \mathrm{g}(\mathbb{H}, \mathbb{R}) \mathbb{S}^{\prime \prime}\right) \wedge$

$\left(\mathrm{h}^{\prime \prime \prime} \mathbb{S}^{\prime} \mathbb{S}^{\prime \prime} \rightarrow \mathrm{h}(\mathbb{H}, \mathbb{R}) \mathbb{S}^{\prime \prime}\right)$

where $\left(\mathrm{p}^{\prime \prime \prime}, \mathrm{g}^{\prime \prime \prime}, \mathrm{h}^{\prime \prime \prime}\right)=\Psi\left(\mathbb{S}^{\prime} \cdot \mathbb{R}(\$ \mathrm{rh})\right)$

4. $\forall \mathbb{S}, \mathbb{S}^{\prime} \cdot \mathrm{g}^{\prime} \mathbb{S} \mathbb{S}^{\prime} \rightarrow \mathbb{S} \cdot \mathbb{R}(\$ \mathrm{ra})=\mathbb{S}^{\prime} \cdot \mathbb{R}(\$ \mathrm{ra})$

5. $\forall \mathbb{S}, \mathbb{S}^{\prime} \cdot \mathrm{h}^{\prime} \mathbb{S} \mathbb{S}^{\prime} \rightarrow \mathbb{S} \cdot \mathbb{R}(\$ r h)=\mathbb{S}^{\prime} \cdot \mathbb{R}(\$ r h)$ we have

$\forall \Psi^{\prime}, \mathbb{H}, \mathbb{R}$.

$\langle[(\mathrm{p}, \mathrm{g}, \mathrm{h}) \rrbracket\rangle\rangle_{\Psi} \Psi^{\prime}(\mathbb{H}, \mathbb{R}) \rightarrow \llbracket \Psi^{\prime}(\mathrm{f}) \rrbracket \Psi^{\prime}(\mathbb{H}, \mathbb{R})$

(In short, the E-TRY rule is derivable from the $\mathrm{J}$ rule).

Proof. Unfolding the definition of the interpretation function, we know that, given

6. $\Psi \subseteq \Psi^{\prime}$

7. $\mathrm{p}(\mathbb{H}, \mathbb{R})$

8. $\operatorname{WFSC}\left(n, \mathrm{~g}(\mathbb{H}, \mathbb{R}), \mathrm{h}(\mathbb{H}, \mathbb{R}), \Psi^{\prime}\right)$

we need to prove

a. $\mathrm{p}^{\prime}(\mathbb{H}, \mathbb{R})$

b. $\operatorname{WFSC}\left(n+1, \mathrm{~g}^{\prime}(\mathbb{H}, \mathbb{R}), \mathrm{h}^{\prime}(\mathbb{H}, \mathbb{R}), \Psi^{\prime}\right)$

The proof of a is trivial (by 1 ). We focus on the proof of $b$.

For all $\mathbb{S}^{\prime}$, if $\mathrm{g}^{\prime}(\mathbb{H}, \mathbb{R}) \mathbb{S}^{\prime}$, by 7 and 2 we know:

9. $\mathbb{S}^{\prime} \cdot \mathbb{R}(\$ \mathrm{ra}) \in \operatorname{dom}\left(\Psi^{\prime}\right)$

10. $\mathrm{p}^{\prime} \mathbb{S}^{\prime}$

11. $\forall \mathbb{S}^{\prime \prime} \cdot \mathrm{g}^{\prime} \mathbb{S}^{\prime} \mathbb{S}^{\prime \prime} \rightarrow \mathrm{g}(\mathbb{H}, \mathbb{R}) \mathbb{S}^{\prime \prime}$

12. $\forall \mathbb{S}^{\prime \prime} \cdot \mathrm{h}^{\prime} \mathbb{S}^{\prime} \mathbb{S}^{\prime \prime} \rightarrow \mathrm{h}(\mathbb{H}, \mathbb{R}) \mathbb{S}^{\prime \prime}$

13. $\operatorname{WFSC}\left(n, \mathrm{~g}^{\prime} \mathbb{S}^{\prime}, \mathrm{h}^{\prime} \mathbb{S}^{\prime}, \Psi^{\prime}\right)$ by C.1 and C.2

For all $\mathbb{S}^{\prime}$, if $\mathrm{h}^{\prime}(\mathbb{H}, \mathbb{R}) \mathbb{S}^{\prime}$,

by $4, \mathbb{R}(\$ r h)=\mathbb{S}^{\prime} \cdot \mathbb{R}(\$ r h)$

by 3 we know:

14. $\mathbb{S}^{\prime} \cdot \mathbb{R}(\$ r h) \in \operatorname{dom}\left(\Psi^{\prime}\right)$ by above equality

15. $\mathrm{p}^{\prime \prime} \mathbb{S}$

16. $\forall \mathbb{S}^{\prime \prime} \cdot \mathrm{g}^{\prime \prime} \mathbb{S}^{\prime} \mathbb{S}^{\prime \prime} \rightarrow \mathrm{g}(\mathbb{H}, \mathbb{R}) \mathbb{S}^{\prime \prime}$

17. $\forall \mathbb{S}^{\prime \prime} \cdot \mathrm{h}^{\prime \prime} \mathbb{S}^{\prime} \mathbb{S}^{\prime \prime} \rightarrow \mathrm{h}(\mathbb{H}, \mathbb{R}) \mathbb{S}^{\prime \prime}$

18. $\operatorname{WFSC}\left(n, \mathrm{~g}^{\prime \prime} \mathbb{S}^{\prime}, \mathrm{h}^{\prime \prime} \mathbb{S}^{\prime}, \Psi^{\prime}\right)$ by C.1 and C.2

19. $\exists m . m<n+1 \wedge \operatorname{WFSC}\left(m, \mathrm{~g}^{\prime \prime} \mathbb{S}^{\prime}, \mathrm{h}^{\prime \prime} \mathbb{S}^{\prime}, \Psi^{\prime}\right)$ by $m=n$.

where $\left(\mathrm{p}^{\prime \prime}, \mathrm{g}^{\prime \prime}, \mathrm{h}^{\prime \prime}\right)=\Psi^{\prime}\left(\mathbb{S}^{\prime} \cdot \mathbb{R}(\$ \mathrm{rh})\right)$

By definition of WFSC, 13 and 19 ,

$\operatorname{WFSC}\left(n+1, \mathrm{~g}^{\prime}(\mathbb{H}, \mathbb{R}), \mathrm{h}^{\prime}(\mathbb{H}, \mathbb{R}), \Psi^{\prime}\right)$

Lemma C.6 (E-Ret) If $\forall \mathbb{S} . p \mathbb{S} \rightarrow g \mathbb{S} \mathbb{S}$, then for all $\Psi, \mathbb{H}$ and $\mathbb{R}$, we have

$$
[[(\mathrm{p}, \mathrm{g}, \mathrm{h})]] \Psi(\mathbb{H}, \mathbb{R}) \rightarrow[[\Psi(\mathbb{R}(\$ \mathrm{ra}))] \Psi(\mathbb{H}, \mathbb{R}) .
$$

That is, the E-RET rule is derivable from an instantiation of the JR rule, where $r_{s}$ is instantiated to \$ra.

Proof. Unfolding the definition of the interpretation function, we know that, given

1. $\mathrm{p} \mathbb{S}$

2. $\operatorname{WFSC}(n, \mathrm{~g} \mathbb{S}, \mathrm{h} \mathbb{S}, \Psi)$

we need to prove

a. $\mathrm{p}^{\prime} \mathbb{S}$

b. $\exists m \cdot \operatorname{WFSC}\left(m, \mathrm{~g}^{\prime} \mathbb{S}, \mathrm{h}^{\prime} \mathbb{S}, \Psi\right)$

where $\left(\mathrm{p}^{\prime}, \mathrm{g}^{\prime}, \mathrm{h}^{\prime}\right)=\Psi(\mathbb{R}(\$ \mathrm{ra}))$.

By 1 . we know $g \mathbb{S} \mathbb{S}$

Then by 2 , we know that either $n=0$ or $n>0$. In the first case $\neg \exists \mathbb{S}^{\prime}$.g $\mathbb{S} \mathbb{S}^{\prime}$, but since $\mathrm{g} \mathbb{S} \mathbb{S}$, this case is vacuous.

Then $n>0$, and we know the following:

- $\mathbb{S} . \mathbb{R}(\$ r a) \in \operatorname{dom}(\Psi)$

- $p^{\prime} \mathbb{S}$ 
- $\operatorname{WFSC}\left(n-1, \mathrm{~g}^{\prime} \mathbb{S}, \mathrm{h}^{\prime} \mathbb{S}, \Psi\right)$

where $\left(\mathrm{p}^{\prime}, \mathrm{g}^{\prime}, \mathrm{h}^{\prime}\right)=\Psi(\mathbb{R}(\$ \mathrm{ra}))$.

By choosing $m=n-1$, we satisfy both $a$ and $b$.

Lemma C.7 (E-Raise) If $\forall \mathbb{S}$.p $\mathbb{S} \rightarrow \mathrm{h} \mathbb{S} \mathbb{S}$, then for all $\Psi, \mathbb{H}$ and $\mathbb{R}$, we have

$$
[[(\mathrm{p}, \mathrm{g}, \mathrm{h})]] \Psi(\mathbb{H}, \mathbb{R}) \rightarrow[[\Psi(\mathbb{R}(\$ \mathrm{rh}))]] \Psi(\mathbb{H}, \mathbb{R}) .
$$

That is, the E-RAISE rule is derivable from an instantiation of the JR rule, where $r_{s}$ is instantiated to $\$$ rh.

Proof. Unfolding the definition of the interpretation function, we know that, given

1. $\mathrm{p} \mathbb{S}$

2. $\operatorname{WFSC}(n, \mathrm{~g} \mathbb{S}, \mathrm{h} \mathbb{S}, \Psi)$ for some $n$. we need to prove

a. $\mathrm{p}^{\prime \prime} \mathbb{S}$

b. $\exists l \cdot \operatorname{WFSC}\left(l, \mathrm{~g}^{\prime \prime} \mathbb{S}, \mathrm{h}^{\prime \prime} \mathbb{S}, \Psi\right)$

where $\left(\mathrm{p}^{\prime \prime}, \mathrm{g}^{\prime \prime}, \mathrm{h}^{\prime \prime}\right)=\Psi(\mathbb{R}(\$ \mathrm{rh}))$.

By 1 . we know $\mathrm{h} \mathbb{S} \mathbb{S}$

Then by 2 , we know that either $n=0$ or $n>0$. In the first case $\neg \exists \mathbb{S}^{\prime} . \mathrm{h} \mathbb{S} \mathbb{S}^{\prime}$, but since $\mathrm{h} \mathbb{S} \mathbb{S}$, this case is vacuous.

Then $n>0$, and we know the following:

- $\mathbb{S} . \mathbb{R}(\$ r h) \in \operatorname{dom}(\Psi)$

- $\mathrm{p}^{\prime \prime} \mathbb{S}$

- $\exists m, m<n \wedge \operatorname{WFSC}\left(n, \mathrm{~g}^{\prime \prime} \mathbb{S}, \mathrm{h}^{\prime \prime} \mathbb{S}, \Psi\right)$

where $\left(\mathrm{p}^{\prime \prime}, \mathrm{g}^{\prime \prime}, \mathrm{h}^{\prime \prime}\right)=\Psi(\mathbb{R}(\$ \mathrm{rh}))$.

By using $\mathrm{m}$ as 1 , we satisfy both a and $\mathrm{b}$. 